Бріологія, ліхенологія та мікологія

\title{
The Second Checklist of Bryobionta of Ukraine
}

\author{
MYKHAILO FEDOSIIOVYCH BOIKO
}

BoIKo M.F. (2014). The Second checklist of Bryobionta of Ukraine. Chornomors ' $k$. bot. z., 10 (4): 426-487. doi:10.14255/2308-9628/14.104/2.

The second checklist of bryophythes of Ukraine is given according to the contemporary taxonomical system in this edition (Checklist first published in 2008). The bryophytes (superdivision Bryobionta) refer to 3 divisions: Anthocerotophyta (4 species), Marchantiophyta (192 species), Bryophyta (653 species). Species are placed alphabetically. Modern Latin names and known synonyms are given to each species. Literature sources on the Ukrainian bryoflora, including those published long ago as well as of the latest decades, especially generalized works, are quoted here. Distribution of all the species both in physiogeographic plains zones and mountain landscapes: zone of mixed coniferousdeciduous forests (Ukraine Polissja), zone of deciduous forests (nemoral zone), the Foreststeppe zone, the Steppe zone (with steppe Crimea), Carpathian mountain region, Crimean mountain region and administrative regions of Ukraine is shown. The indications about species which were not presented in the first Ukrainian Checklist of Briobionta (2008), questionable species and species that were excluded from Ukrainian bryoflora are given.

Key words: Bryobionta, Anthocerotophyta, Marchantiophyta, Bryophyta, Checklist, Ukraine

БОйКО М.Ф. (2014). Другий чекліст мохоподібних України. Чорноморськ. бот. ж., 10 (4): 426-487. doi:10.14255/2308-9628/14.104/2.

Другий чекліст мохоподібних України складено відповідно до сучасної таксономічної системи бріофітів (перший чекліст опубліковано у 2008 році). Мохоподібні (надвідділ Bryobionta) відносяться до 3 відділів: Anthocerotophyta (4 види), Marchantiophyta (192 вид), мохи (653 види). Види у відділах розташовані в алфавітному порядку. Для кожного виду вказані сучасні латинські назви, найбільш відомі синоніми, поширення у фізико-географічних рівнинних зонах: зоні мішаних хвойно-широколистяних лісів (Українське Полісся), зоні широколистяних лісів (неморальна зона), лісостеповій зоні та степовій зоні (в т.ч у кримських степах) та у гірських ландшафтних країнах Карпатська гірська країна (Українські Карпати), Кримська гірська країна та поширення видів в адміністративних областях України. Наводяться вказівки щодо видів, які не вказувалися у першому Чеклісті мохоподібних України (2008), а також сумнівні види та види, що виключені з бріофлори України.

Ключові слова: Bryobionta, Anthocerotophyta, Marchantiophyta, Bryophyta, чекліст, Україна

БОЙКО М.Ф. (2014). Второй чеклист мохообразных Украины. Черноморск. бот. ж., 10 (4): 426-487. doi:10.14255/2308-9628/14.104/2.

Второй чеклист мохообразних Украины составлен в соответствии с современной таксономической системой бриофитов (первый чеклист опубликован в 2008 году). Мохообразные (надотдел Bryobionta) относятся к 3 отделам: Anthocerotophyta (4 вида), Marchantiophyta (192 вид), мхи (653 вида). Виды в отделах расположены в алфавитномм порядке. Для каждого вида указаны современные латинские названия, наболее известные синонимы, распространение в физико-географических равнинных зонах: (зоне смешанных хвойно-широколиственых лесов (Украинское Полесье), зоне широколиственных лесов (неморальная зона), лесостепной и степной зоне и в горных ландшафтных странах (Карпатская горная страна (Украинские Карпаты), Крымская горная страна и распросторанение видов в административных областях Украины.

(C) Mykhailo F. Boiko

Чорноморськ. бот. ж., 10 (4): 426-487. 
Приводятся указания относительно видов, которые не указывались в первом Чеклисте мохообразных Украины (2008), а также сомнительные и исключенные из бриофлоры Украины виды.

Ключевые слова: Bryobionta, Anthocerotophyta, Marchantiophyta, Bryophyta, чеклист, Украина

\section{Introduction}

Recently bryologists from all over the world have been intensively developing the issue of taxonomy and phylogeny of bryobionts (mosses). Materials on these issues are presented in many works [YATSENTYUK et al., 2004; BAKALIN, 2005; HE-NYGREN et al., 2006; HeNTSCHEL et al., 2007; ROO DE et al., 2007; CrANDALL-STOTLER et al., 2008, 2009; GofFinet, BuCK, SHAW, 2008; Hill et al., 2006, 2008; KonstantinOva et al., 2009; Konstantinova NA \& Vilnet AA, 2009; SÖDERSTRÖM, De RoO, HedD., 2010; Rubasinghe et al., 2011 a, b; VILNET et al. 2011; VILNET et al., 2012; VÁÑA et al., 2012; FELDBERG et al., 2013; VÁÑA et al., 2013 a, b; CHECKLIST..., 2014, etc.].

The use of phylogenomics and new views on paleobryological materials made it possible to reflect the phylogeny of bryophytes and view their phylogenetic system more accurately [BUCK, GOFFINET \& SHAW, 2000; GOFFINET, BUCK, 2004; TSUBOTA et al., 2002; COX \& HedDerson, 2003; GRUNDMANN et al., 2006; HedenÄs, 2006; HenTSCHEL et al., 2007; RENZAGLiA et al., 2008; OlSSON et al., 2011]. And review applies to the system as a whole and individual departments, classes, families, genera, species aggregates as well with justification of the places of each taxon in the system [LEWINSKY, 1993; ZANDER, 1993; Greven, 1995; Blom, 1996; Munoz, PAndo, 2000; HedenÄs, 2001; Ignatov, HutTunen, 2002; POTEMKIN, 2002; VANDERPOORTEN et al., 2002; WERNER et al., 2002, 2004, 2005, 2007, 2013; HutTunEN, 2004; HutTunEN, IGNATOV, 2004, 2010; SCHUMACKER, VÁŇA, 2005; Holyoak, Pedersen, 2007; SeneCA, SÖDERSTRÖM, 2009; VANDERPOORTEN, HedEnÄS, 2009; HolyoAK, 2010; Ros, HerRnSTADT, 2010; Vilnet et al., 2010; PlaŠEK, et al., 2011; BAKALIN, VILNET, 2012 etc.]. This takes into account many changes in the names of taxa that occurred in monographic descriptions of many genera, families and other taxa and which are used in various lists of bryoflora and bryophyte checklists [IGNATOV, AFONINA, 1992, GAPON, 1998, 2011; CROSBY et al., 1999; VIRCHENKO, VÁŇA, 2000; SÖDERSTRÖM, URMI, and VÁŇA, 2002; Kučera, VÁŇA, 2003; OCHYRA, ZARNOWIEC, BEDNAREK-OCHYRA,2003; GOFFINET et al., 2004; Boiko, 2008, 2011 a, b; Hedenas, Rosborg, 2008; Bell, Hyvonen, 2010; KopOnEn, IsOvitTA, 2010; FeldBerg et al., 2010; Crosby, BuCK, 2011; Ros et al., 2008, 2013; CHECKLIST..., 2014, etc.]. Thereby, it should be noted that the expression of bryophytes system is changing very quickly.

The second edition of the checklist included materials on bryophytes, which appeared after the publication of the first "Checklist of bryophytes of Ukraine" [BOIKO, 2008].

These recent data about the new species from different regions of Ukraine, new to science species and types were missed for different reasons during preparation of the first checklist. Also changes in species composition that occurred as a result of new views of the author and other bryologists on taxonomy and the herbarium specimens study using modern techniques were taken into account [VIRCHENKO, OrLOV, 2011; GAPON, 2011; NYPORKO, PARTYKA, 2011; BOIKO, 2009 a,b,c, 2012; Ros et al., 2013; BARSUKOV, 2014, 2015; BOROVICHOV, NYPORKO, 2014 etc.].

Till 15.12.2014 Bryophyte flora of Ukraine consists of 849 species, 256 genera, 83 families, 29 orders, 8 classes, including: Anthocerotophyta -4 species, 2 genera, 1 family, 1 order, class 1; Marchantiophyta - 192 species, 76 genera, 41 family, 10 orders, 2 classes; Bryophyta - 653 species, 196 genera, 49 families, 19 orders, 5 classes.

The system of bryophytes taxa of Ukraine given in the checklist corresponds to the author's view about their phylogeny and taxonomy, which are based on the materials of the 
authorsmentioned above [HILL et al., 2006, 2008 et al.; BOIKO, 2008; BRYOPHYTE BIOLOGY, 2008; Crandall-Stotler et al., 2008, 2009; Goffinet, Buck, Shaw, 2008; RenZAGLia et al., 2008; KONSTANTINOVA et al., 2009] and on the results of the world modern bryological achievements.

\section{THE SYSTEM OF TAXA OF SUPERDIVISIO BRYOBIONTA UKRAINE}

(superdivisio, divisio, classis, ordo, familia, genus)

\section{SUPERDIVISIO: BRYOBIONTA}

\section{Divisio: ANTHOCEROTÓPHYTA Rothm.}

Class: ANTHOCEROTÓPSIDA de Bari ex Jancz.

Order: Anthocerotàles Limpr.

Anthóceros, L., Phaeóceros Prosk.

Anthocerotàceae Trevis.

Divisio: MARCHANTIÓPHYTA Stotler \& Crand.-Stotl.

Class: HAPLOMITRIÓPSIDA Stotler \& Crand.-Stotl.

Haplomítrium Nees

Order: Calobryàles Hamlin

Haplomitriàceae Dкdксйk

Class: MARCHANTIÓPSIDA Cronquist, Takht. \& W. Zimm.

Order: Blasiàles Stotler \& Crand.-Stotl.

Blàsia L.

Blasiàceae H. Klinggr.

Order: Lunulariàles D.G.Long.

Lunulariàceae H.Klinggr.

Lunulària Adans.

Order: Marchantiàles Limpr.

Marchantiàceae Lindl.

Marchàntia L., Préissia Corda

Aytoniàceae Cavers

Asterélla P.Beauv., Mánnia Opiz, Rebóulia Raddi

Clevéa Lindb., Sautéria Nees

Cleveàceae Cavers

Conocéphalum Hill

Conocephalàceae Müll.Frib. ex Grolle

Oxymitra Bisch. ex Lindenb.

Oxymitraceae Mull. Frib. Ex Grolle

Ricciàceae Rchb.

Ríccia L., Ricciocórpos Corda

Targioniàceae Dumort.

Targiónia L.

Class: JUNGERMANNIÓPSIDA Stotler \& Crand.-Stotl.

Order: Pelliàles Xiao L.He, Juslén, Ahonen, Glenny \& Piippo

Péllia Raddi

Pelliàceae H.Klinggr. 
Order: Fossombroniàles Schljakov

Fossombrónia Raddi

Fossombroniàceae Hazsl.

Order: Pallaviciniàles W.Frey \& M.Stech

Moerckiàceae Stotler \& Crand.-Stotl.

Moérckia Gottsche

Pallavicíinia Gray

Pallaviciniàceae Mig.

Order: Metzgeriàles Chalaud

Metzgeriàceae H.Klinggr.

Metzgéria Raddi

Aneuràceae H.Klinggr.

Anéura Dumort., Riccàrdia Gray

Order: Porellàles Schljakov

Porellàceae Cavers

Porélla L.

Radulàceae Müll.Frib.

Ràdula Dumort.

Frullánia Raddi

Frullaniàceae Lorch

Lejeuneàceae Cavers

Cololejéunea (Spruce) Schiffn., Lejéunea Lib.

Order: Ptilidiàles Schljakov

Ptilidiàceae H.Klinggr.

Ptilídium Nees

Order: Jungermanniàles H.Klinggr.

Pseudolepicoleàceae Fulford \& J.Taylor

Blepharostóma (Dumort.) Dumort.

Trichocólea Dumort.

Trichocoleàceae Nakai

Lepidoziàceae R.M.Schust.

Bazzània Gray, Kúrzia G.Martens, Lepidózia (Dumort.) Dumort.

Chilóscyphus Corda

Lophocoleàceae Vanden Berghen

Lophocólea (Dumort.) Dumort.

Plagiochilàceae Müll.Frib. \& Herzog

Plagióchila (Dumort.) Dumort.

Jamesoniellàceae Xiao L.He, Juslén, Ahonen, Glenny \& Piippo

Pedinophýllum (Lindb.) Lindb., Syzigiélla Spruce

Cephaloziàceae Mig.

Cephalózia (Dumort.) Dumort., Nowéllia Mitt., Pleurocládula (Hook.) Grolle

Odontoschismatàceae (Grolle) Schljakov

Cladopodiélla H.Buch., Odontoschísma (Dumort.) Dumort.

Cephaloziélla (Spruce) Schiffn.

Cephaloziellàceae Douin

Scapaniàceae Mig.

Diplophýllum (Dumort.) Dumort., Lophózia (Dumort.) Dumort., Lophoziópsis Konstant. \& Vilnet, Scapània (Dumort.) Dumort., Tritomària Schiffn. ex Loeske 
Trilophózia (R.M. Schust.) Bakalin

$$
\text { Lophoziaceae Schleich. }
$$

Anastrophyllàceae L. Söderstr., De Roo \& Hedd.

Anastrépta (Lindb.) Schiffn., Anastrophýllum (Spruce) Steph., Barbilophózia Loeske, Crossocàlyx Meyl., Gymnocólea (Dumort.) Dumort., Isopàches H.Buch, Neoorthocàulis L.Söderstr., De Roo \& Hedd., Schljakoviànthus Konstant. \& Vilnet, Sphenolóbus (Lindb.) Berggr.

$$
\text { Myliàceae Schljakov }
$$

Leiomýlia J.J. Engel \& Braggins, Mýlia Gray

Calypogéia Raddi

$$
\text { Calypogeiàceae Arnell }
$$

$$
\text { Jungermanniàceae Reichenbach }
$$

Jungermànnia L., Lióchlaena Nees, Mesoptýchia (Lindb.) A.Evans, Schistochilópsis (N. Kitag.) Konstant.

$$
\text { Endogemmatàceae Konstant., Vilnet \& Troitsky }
$$

Endogémma Konstant., Vilnet \& Troitsky

Solenostomatàceae Stotler \& Crand.-Stotl.

Nàrdia Gray, Solenostóma Mitt.

Geocalycàceae H.Klinggr.

Geocàlyx Nees, Harpànthus Nees

$$
\text { Antheliàceae R.M.Schust. }
$$

Anthélia (Dumort.) Dumort.

Gymnomitriàceae H.Klinggr.

Gymnomitrion Corda, Marsupélla Dumort.

\section{Divisio: BRYÓPHYTA}

\section{Class: SPHAGNÓPSIDA Ochyra}

Order: Sphagnáles Limpr.

Sphágnum L.

Sphagnáceae Dumort.

Class: ANDREAEÓPSIDA Rothm.

Order: Andreaeáles Limpr.

Andreaeáceae Dumort.

Andreaéa Hedw.

\section{Class: POLYTRICHÓPSIDA Doweld}

Order: Polytricháles M. Fleisch.

Polytricháceae Schwägr.

Átrichum P. Beauv., Oligótrichum Lam. \& DC., Pogonátum P.Beauv., Polytrichástrum G. L.Sm., Polýtrichum Hedw.

Class: TETRAPHIDÓPSIDA Goffinet \& W.R.Buck

Order: Tetraphidáles M. Fleisch.

Tetraphidáceae Schimp.

Tetráphis Hedw., Tetrodóntium Schwägr. 


\section{Class: BRYÓPSIDA Rothm.}

Order: Buxbaumiáles M. Fleisch.

Buxbáumia Hedw.

Buxbaumiáceae Schimp.

Order: Diphysciáles M. Fleisch.

Diphýscium Mohr

Diphysciáceae M. Fleisch.

Order: Timmiáles Ochyra

Timmiáceae Schimp.

Tímmia Hedw.

Order: Encalyptáles Dixon

Encalyptaceae Schimp.

Encalýpta Hedw.

Order: Funariáles M.Fleisch.

Funariáceae Schwägr.

Entósthodon Schwägr. Funária Hedw., Physcomitrélla Bruch \& Schimp., Physcomitrium (Brid.) Brid., Pyramidula Brid.

Order: Grimmiáles M. Fleisch.

Grimmiáceae Arn. Schimp.

Coscínodon Spreng., Grímmia Hedw. , Racomitrium Brid., Schistídium Bruch \&

Campylostélium Bruch \& Schimp.

Ptychomitriáceae Schimp.

Seligeriáceae Schimp.

Blíndia Bruch \& Schimp., Brachydóntium Fürnr., Seligéria Bruch \& Schimp.

Order: Dicranáles H. Philib. ex M. Fleisch.

Fissidens Hedw.

Fissidentáceae Schimp.

Ditricháceae Limpr.

Cerátodon Brid, Cleistocarpídium Ochyra \& Bednarek-Ochyra, Distíchium Bruch \& Schimp., Dítrichum Hampe, Pleurídium Rabenh., Saelània Lindb., Tríchodon Schimp.

Tremátodon Michx.

Bruchiaceae Schimp.

Schistostéga Mohr

Schistostegáceae Schimp. 
Rhabdoweisiáceae Limpr.

Amphídium Schimp., Arctóa Bruch \& Schimp., Cynodóntium Bruch \& Schimp., Dichodóntium Schimp., Dicranowéisia Milde, Kiaéria Hag., Oncóphorus (Brid.) Brid., Rhabdowéisia Bruch \& Schimp.

Dicranáceae Schimp.

Cnéstrum Hag., Dicranélla (Müll.Hal.) Schimp., Dicránum Hedw., Paraleucóbryum (Limpr.) Loeske, Pseudephémerum (Lindb.) I. Hag.

Leucobryáceae Schimp.

Campylópus Brid., Dicranodóntium Bruch \& Schimp., Leucóbryum Hampe

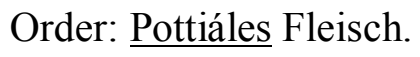

Pottiáceae Schimp.

Acáulon Müll. Hal., Aloína Kindb., Anoectángium Schwaegr., Bárbula Hedw., Bryoerythrophýllum P.C.Chen, Cinclídótus P. Beauv., Crossídium Jur., Didýmodon Hedw., Ephémerum Hampe, Eucládium Bruch \& Schimp., Gymnóstomum Nees \& Hornsch., Gyrowéisia Schimp., Hennediélla Paris, Hymenostýlium Brid., Lazarénkia Boiko, Micróbryum Schimp., Molendóa Lindb., Oxýstegus (Broth.) Hilp., Pháscum Hedw., Pseudocrossídium R.S. Williams, Pterygonéurum Jur., Syntríchia Brid., Tortélla (Lindb.) Limpr., Tórtula Hedw., Trichostomum Bruch, Weíssia Hedw.

Order: Splachnáles Ochyra

Splachnáceae Grev. \& Arn.

Spláchnum Hedw. Taylória Hook., Tetráplodon (Bruch \& Schimp.) Wilson

Meesiáceae Schimp.

Amblýodon P. Beauv., Leptóbryum (Bruch \& Schimp.) Wilson, Meésia Hedw., Paludélla Brid.

Order: Bryales Limpr.

Bryáceae Schwaegr.

Anomóbryum Schimp., Brýum Hedw., Imbribryum N. Pedersen, Ptychostomum Hornsch., Rhodóbryum (Schimp.) Limpr.

Mniáceae Schwägr.

Cinclídium Sw., Mníum Hedw., Plagiómnium T. Kop., Póhlia Hedw., Pseudóbryum (Kindb.) T. Kop., Rhizómnium (Broth.) T. Kop.

Bartramiáceae Schwägr.

Bartrámia Hedw., Philónotis Brid., Plagiópus Brid.

Order: Orthotricháles Dixon

Orthotricháceae Arn.

Nyholmiella Holmen \& E. Warncke Orthótrichum Hedw. Ulóta D. Mohr Zýgodon Hook. \& Taylor

Order: Hedwigiáles Ochyra

Hedwigiáceae Schimp.

Hedwígia P.Beauv. 
Order: Rhizogoniales Goffinet \& W.R. Buck

Aulacómnium Schwaegr.

Aulacomniáceae Schimp.

Order: Hookeriáles M. Fleisch.

Hookeriáceae Schimp.

Hookéria Sm.

Order: Hypnáles (Fleisch.) W. R. Buck \& Vitt

Fontinaláceae Schimp.

Dichelýma Myrin, Fontinális Hedw.

Climácium F.Weber \& D.Mohr

Climaciáceae Kindb.

Amblystegiáceae Kindb.

Amblystégium Schimp., Anacámptodon Brid., Campyliadélphus (Kindb.) R. S. Chopra, Campylidium (Kindb.) Ochyra, Campýlium (Sull.) Mitt., Conárdia H. Robins., Cratonéuron (Sull.) Spruce, Drepanócladus (Müll.Hall.) G. Roth, Hygroamblystégium Loeske, Hygróhypnum Lindb., Leptodíctyum (Schimp.) Warnst., Myrinia Schimp., Palustriélla Ochyra, Saniónia Loeske, Scorpídium (Schimp.) Limpr.

Calliergonáceae (Kanda) Vanderp., Hedenäs, C. J. Cox \& A. J. Shaw Loeske

Calliérgon (Sull.) Kindb., Hamatocáulis Hedenäs, Straminérgon Hedenäs, Warnstórfia

Helódium Warnst.

Helodiáceae Ochyra

Leskeáceae Schimp.

Haplocládium (Müll. Hall) Müll.Hall., Lescúraea Bruch \& Schimp., Léskea Hedw., Pseudoleskeélla Kindb.

Thuidiáceae Schimp

Abietinélla Müll. Hall., Pelékium Mitt.,Thuídium Bruch. \& Schimp.

Brachytheciaceae Schimp.

Brachytheciástrum Ignatov \& Huttunen, Brachythécium Schimp., Breidléria Loeske, Callicládium H.A.Crum, Campylophýllum (Schimp.) M. Fleisch., Cirriphýllum Grout, Eurhynchiástrum Ignatov \& Huttunen, Eurhýnchium Bruch. \& Schimp., Homalothécium Schimp., Kindbérgia Ochyra, Oxyrrhýnchium (Schimp.) Warnst., Palamocládium Müll. Hall., Plasteurhýnchium Broth., Pseudoscleropódium (Limpr.) M. Fleisch., Rhynchostegiélla (Schimp.) Limpr., Rhynchostégium Bruch. \& Schimp., Scorpiúrium Schimp.Sciuro-hýpnum (Hampe) Hampe,Tomenthýpnum Loeske

Hypnáceae Schimp.

Calliergonélla Loeske, Herzogiélla Broth., Homomállium (Schimp.) Loeske, Hýpnum Hedw., Isopterygiópsis Z. Iwats., Orthothécium Schimp., Platydictya Berk., Pseudotaxiphýllum Z. Iwats., Ptílium De Not., Pyláisia Schimp., Taxiphýllum M. Fleisch.

Pterigynándraceae Schimp.

Heterocládium Bruch \& Schimp., Myurélla Schimp., Pterigynándrum Hedw. 
Hylocomiáceae M. Fleisch.

Ctenídium (Schimp.) Mitt., Hylocomiástrum Broth., Hylocómium Bruch \& Schimp., Loeskeóbryum Broth., Pleurózium Mitt.,Rhytidiadélphus (Limpr.) Warnst.

Rhytídium (Sull.) Kindb.

Rhytidiáceae Broth.

Plagiotheciaceae (Broth.) Fleisch.

Plagiothécium Schimp.

Éntodon Müll.Hal.

Entodontáceae Kindb., nom. cons

Pylaisiadelpháceae Goffinet \& W. R. Buck

Heterophýllium (Schimp.) Kindb., Platygýrium Schimp.

Leucodontáceae Schimp

Antitríchia Brid., Leucódon Schwägr.

Neckeráceae Schimp

Alleniélla S.Olsson, Enroth \& D.Quandt, Homália (Brid.) Bruch \& Schimp., Néckera Hedw.,

Thamnóbryum Nieuwl.

Leptódon D. Mohr

Leptodontáceae Schimp

Lembophylláceae Broth.

Isothécium Brid.

Anomodontáceae Kindb

Anómodon Hook. \& Taylor

CONVENTIONAL SIGNS

Administrative oblasts of Ukraine

Ch - Chernihiv oblast'
Ck - Cherkassy oblast'
Cv - Chernivtsi oblast'
Dn - Dnipropetrovs'k oblast'
Do - Donets'k oblast'
If - Ivano-Frankivs'k oblast'
Ka - Kharkiv oblast'
Ke - Kherson oblast'
Ki - Kirovohrad oblast'
Km - Khmelnitsky oblast'
Kr - AR Crimea
Ky - Kyiv oblast'
Lu - Lugans'k oblast'
Lv - Lviv oblast'
My - Mycolaiv oblast'
Od - Odessa oblast'
Po - Poltava oblast'
Ri - Rivne oblast'

Sk - Sakarpattya oblast'

Sp - Saporizhzhya oblast'

Su - Sumy oblast'

Te - Ternopil' oblast'

V1 - Volyn' oblast'

Vn - Vinnitsya oblast'

Zh - Zhytomyr oblast'

Physiogeographic plains zones and

mountainous landscapes countries of Ukraine

$\mathrm{MF}$ - Zone of mixed coniferous-deciduous forests

DF - Zone of deciduous forests (nemoral zone)

FSZ - The Forest-steppe zone

SZ - The Steppe zone (with steppe

Crimea) (Sz)

CAM - Carpathian mountain region

CRM - Crimean mountain regio 


\section{LIST OF TAXA}

\section{ANTHOCEROTOPHYTA}

Anthóceros agréstis Paton (A. punctatus L. var. cavernosus (Nees) Gott., Lindenb. et Nees) [MF, DF, FSZ], Ch, Ck, Ky, Lv, Su.

Anthóceros punctátus L. s. str. - [CAM, MF, DF, FSZ], Ch, Ck, If, Lu, Su.

Phaeóceros caroliniánus (Michx.) Prosk. (P. laevis subsp. carolinianus (Michx.) Prosk.) [MF], Ky.

Phaeóceros laévis (L.) Prosk. (Anthoceros laevis L. ) - [CAM, MF, DF, FSZ, SZ], Ch, Ck, Dn, If, Ky, Lu, Su, Te.

\section{MARCHANTIOPHYTA}

Anastrépta orcadénsis (Hook.) Schiffn. - [CAM], If, Sk.

Anastrophýllum micháuxii (F. Weber) H. Buch. - [CAM], Cv, If, Lv, Sk.

Anéura pínguis (L.) Dum. (Riccardia pinguis (L.) S.Gray) - [CAM, MF, DF, FSZ, $\mathrm{CRM}$ ], Ch, Ck, If, Ka, Ki, Ky, Lv, Su, Vn, Zh.

Anthélia juratzkána (Limpr.) Trevis. (Jungermannia juratzkana Limpr.) - [CAM], If, Sk.

Asterélla lindenbergiána (Corda ex Nees) Lindb. ex Arnell. - [CAM], Sk.

Barbilophózia barbáta (Schmid. ex Schreb.) Loeske - [CAM, MF, DF, FSZ, CRM], $\mathrm{Ch}, \mathrm{Ck}, \mathrm{Cv}$, If, Kr, Lv, My, Ri, Vn, Zh.

Barbilophozia hátcheri (A. Evans) Loeske - [CAM], If, Sk.

Barbilophozia lycopodióides (Wallr.) Loeske - [CAM], If, Sk.

Barbilophozia sudetica (Nees ex Huebener) L. Soderstr., De Roo et Hedd. (Lophozia sudetica (Nees ex Huebener) Grolle, L. alpestris (Schleich.) Evans.). - [CAM], If, Lv, Sk.

Bazzánia tricrenáta (Wahlenb.) Lindb. - [CAM], If, Sk,

Bazzania trilobáta (L.) Gray - [CAM, DF], Cv, If, Lv, Sk.

Blásia pusílla L. - [CAM, MF, DF,FSZ], Ch, Ck, Cv, If, Kr, Ky, Lv, Sk, Zh.

Blepharostóma trichophýllum (L.) Dumort. - [CAM, MF, DF], Cv, Ch, If, Lv, Te, Sk, Zh.

Calypogéia azúrea Stotler \& Crotz (C. trichomanis (L.) Corda) - [CAM, MF, DF], Cv, If, Ky, Lv, Sk, Zh.

Calypogeia físsa (L.) Raddi - [MF], Ky.

Calypogeia integristípula Steph. (C. meylanii Steph., C. neesiana var. meylanii (Buch) Schust.) - [CAM], Cv, If.

Calypogeia muehlleriána (Schiffn.) Müll. Frib. - [CAM, MF], If, Ky, Sk.

Calypogeia neesiána (C. Massal. \& Carestia) Müll. Frib. - [CAM, MF], Cv, If, Ky, Lv, $\mathrm{Sk}, \mathrm{Su}, \mathrm{Vl}, \mathrm{Zh}$.

Calypogeia sphagnícola (Arnell \& J.Perss.) Warnst. \& Loeske - [CAM], If, Sk.

Calypogeia suécica (Arnell \& J.Perss.) Müll. Frib. - [CAM], Cv, If, Lv, Sk.

Cephalózia ambígua C. Massal. - [CAM], If, Sk.

Cephalozia bicuspidáta (L.) Dumort. - [CAM, MF, DF, FSZ], Ch, Ck, Cv, If, Ky, Lv, Sk, Te, Zh.

Cephalozia catenuláta (Huebener) Lindb. (C. reclusa (Tayl.) Dum.) - [CAM, MF, DF], If, Lv, Sk, Zh

Cephalozia connívens (Dicks.) Lindb.- [CAM, MF, DF, FSZ], If, Lv, Sk, Zh.

Cephalozia lammersiána (Huebener) Spruce (Cepalozia bicuspidata (L.) Dumort. subsp. lammersiana (Huebener) R.M.Schust.) - [CAM, MF], If, Lv, Sk, Zh.

Cephalozia leucánta Spruce - [CAM, MF, DF], Lv, Zh.

Cephalozia loitlesbérgeri Schiffn. - [CAM], If. 
Cephalozia lunulifólia (Dumort.) Dumort. (C. media Lindb.) [CAM, MF], Ch, Ck, If, Ky, Lv, Sk, Zh.

Cephalozia pléniceps (Austin) Lindb. - [CAM, MF, DF], If, Lv, Vl, Sk, Zh.

Cephaloziélla divaricáta (Sm.) Schiffn. (C. starkei (Funck) Schiffn.) - [CAM, MF, DF, FSZ, SZ, CRM], Ch, Do, Ke, Kr, Lv, Lu, Sk, Su.

Cephaloziella elachísta (J. B. Jack ex Gottsche \& Rabenh.) Lacout. - [CAM], If.

Cephaloziella élegans (Heeg) Schiffn.- [DF], Lv.

Cephaloziella grimsulána (J. B. Jack ex Gottsche \& Rabenh.) Lacout.) - [CRM], Kr.

Cephaloziella hampeána (Nees) Schiffn. - [MF, DF, FSZ, CRM], Ch, Ka, Kr, Ky, Lv, $\mathrm{Su}$.

Cephaloziella rubélla (Nees) Warnst. - [CAM, MF, DF, FSZ, CRM], If, Ka, Kr, Lv, Su, Zh.

Cephaloziella spinígera (Lindb.) Jorg. (Cephaloziella subdentata Warnst.) - [CAM, $\mathrm{MF}], \mathrm{Ch}$, If, Lv, Zh.

Chilóscyphus frágilis (Roth.) Schiffn. (C. pallescens var. fragilis (A. Roth) K. Müll.) [CAM, MF, FSZ], If, Ky, Po, Sk, Zh.

Chiloscyphus palléscens (Ehrh. ex Hoffm.) Dumort. (C. polyanthus var. pallescens Hartm.) - [CAM, MF, DF, FSZ, CRM], Ck, Cv, If, Ki, Km, Kr, Ky, Lv, Po, Ri, Sk, Su, Te, Zh.

Chiloscyphus polyánthos (L.) Corda - [CAM, MF, DF], Ch, Cv, If, Ky, Lv, Sk, Te, Vl, Zh.

Chiloscyphus rivularis (Schrad.) Hazsl. (C. polyanthus var. rivularis Schrad.) Lindb. et Arnell.) - [CAM, CRM], If, Kr, Lv, Sk.

Cladopodiélla flúitans (Nees) H. Buch (Cephalozia fluitans (Nees) Spruce) - [CAM, MF, DF], If, Lv, Sk, Zh.

Clévea hyalina (Sommerf.) Lindb. (Athalamia hyalina (Sommerf.) S. Hatt.) - [CAM, CRM], If, $\mathrm{Kr}$.

Clevea spathysii (Lindenb.) K.Müll. (Athalamia spathysii (Lindenb.) S. Hatt.) - [CAM], If.

Cololejéunea calcárea (Lib.) Schiffn. - [CAM, CRM], If, Kr, Sk.

Cololejeunea rossetiána (C. Massal) Schiffn. - [CAM, DF, CRM], Km, Kr, Sk.

Conocéphalum cónicum (L.) Underw. - [CAM, MF, DF, FSZ], Ck, Cv, If, Km, Ky, Lv, Ri, Sk, Te, $\mathrm{Vn}, \mathrm{Zh}$.

Conocephalum salebrosum Szweyk., Buczk. et Odrzyk. - [DF], Lv.

Crossocàlyx helleriánum (Nees) Meyl. (Anastrophyllum hellerianum (Nees ex Lindenb.) R. M. Schust.) - [CAM, MF], Cv, If, Lv, Zt.

Diplophýllum álbicans (L.) Dumort. - [CAM, MF], Cv, If, Lv, Sk.

Diplophyllum obtusifólium (Hook.) Dumort. - [CAM, MF, DF], If, Lv, Sk, Te.

Diplophyllum taxifólium (Wahlenb.) Dumort. - [CAM], If, Sk.

Endogémma caespitícia (Lindenb.) Konstant., A.Vilnet et A.V.Troitsky (Solenostoma caespiticium (Lindenb.) Steph, Jungermannia caespiticia Lindenb.) - [CAM, MF, DF], $\mathrm{Cv}, \mathrm{Ky}, \mathrm{Lv}$.

Fossombrónia foveoláta Lindb. - [MF,SZ], Dn, Ka, Ky, Vl, Zh.

Fossombronia pusílla (L.) Nees - [CAM, MF], Cv, Zh.

Fossombronia wondraczékii (Corda) Lindb. - [CAM, MF, DF, FSZ], Ch, Ck, If, Ka, $\mathrm{Ky}, \mathrm{Lv}, \mathrm{Su}, \mathrm{Zh}$.

Frullánia dilatáta (L.) Dumort. - [CAM, MF, DF, FSZ, SZ, CRM], Ch, Ck, Cv, Dn, Do, If, Ke, Ky, Ki, Km, Kr, Lv, Lu, My, Od, Po, Sk, Sp, Su, Te, Vl, Vn, Zh.

Frullania fragilifólia (Taylor) Gottsche, Lindenb. \& Nees - [CAM, MF], Cv, Sk, Zh.

Frullania jáckii Gottsche -[CAM], Sk.

Frullania tamarísci (L.) Dumort. (F. nervosa Mont.) - [CAM, MF, CRM], Cv, If, Kr, Lv, Sk, Zh. 
Geocályx graveólens (Schrad.) Nees - [CAM, MF, FSZ], If, Sk, Te, Vl, Zh.

Gymnocólea infláta (Huds.) Dumort. - [CAM], Cv, If, Lv.

Gymnomítrion adústum Nees (Marsupella adusta (Nees emend.Limpr.) Spruce) [CAM], Sk.

Gymnomitrion concinnátum (Lightf.) Corda - [CAM], If, Sk.

Gymnomitrion corallioídes Nees - [CAM], Sk.

Haplomítrium hoókeri (Sm.) Nees - [CAM], If.

Harpánthus flotoviánus (Nees) Nees - [CAM], If, Sk.

Harpanthus scutátus (F.Weber \& Mohr) Spruce - [CAM], Cv, If, Lv, Sk.

Isopàches bicrenátus (Schmidel ex Hohhm.) H. Buch (Lophozia bicrenata (Schmid. ex Hoffm.) Dumort.) - [CAM, MF, DF, FSZ], Lv, Sk, Su, Vn, Zh.

Jungermánnia atrovírens Dumort. (Haplozia atrovirens (Schleich.) Dum.) - [CAM, $\mathrm{CRM}$ ], $\mathrm{Cv}$, If, Kr, Lv, Sk.

Jungermannia púmila With. (Haplozia pumila (With.) Dum., Solenostoma pumila (With.) K. Müll.) - [CAM], Cv, If, Sk.

Kúrzia pauciflóra (Dicks.) Grolle (Telaranea setacea (Weber) K. Müll.) - [CAM, MF], If, Sk, Zh.

Leiomýlia anómala (Hook.) J.J. Engel et Braggins (Mylia anomala (Hook.) Gray Gray) - [CAM, MF], If, Ri, Sk, Zh.

Lejéunea cavifólia (Ehrh.) Lindb. - [CAM, MF, DF, FSZ, CRM], Cv, If, Kr, Lv, Sk, Zh. Lepidózia réptans (L.) Dumort. - [CAM, MF, DF, FSZ], Cv, Ch, If, Ky, Lv, Sk, Te, Vn, Vl, Zh.

Liochlaéna lanceoláta Nees (Jungermannia leiantha Grolle, J. lanceolata L., Solenostoma lanceolata Steph.) - [CAM, MF, DF, FSZ], Cv, If, Ky, Lv, Ri, Sk, Te, Zh.

Liochlaena subuláta (A.Evans) Schljakov (Jungermannia subulata A. Evans) - [MF], $\mathrm{Zh}$.

Lophocólea bidentáta (L.) Dumort (Lophocolea coadunata (Sw.) Mont., Chiloscyphus coadunatus (Sw.) Engel \& Schust.) - [CAM, MF, DF, FSZ, SZ, CRM], Ch, Ck, Cv, Dn, If, $\mathrm{Kr}, \mathrm{Ky}, \mathrm{Lv}, \mathrm{Lu}, \mathrm{Po}, \mathrm{Sk}, \mathrm{Sp}, \mathrm{Su}, \mathrm{Te}, \mathrm{Zh}$.

Lophocolea heterophýlla (Schrad.) Dumort. - [CAM, MF, DF, FSZ, SZ, CRM], Ch, Ck, $\mathrm{Cv}$, Dn, Do, If, Ka, Km, Kr, Ky, Lu, Lv, Od, Po, Sk, Su, Te, Vl, Vn, Zh.

Lophocolea mínor Nees - [CAM, MF, DF, FSZ, SZ, CRM], Ch, Ck, Cv, If, Ka, Km, Kr, $\mathrm{Ky}, \mathrm{Lu}, \mathrm{Lv}, \mathrm{My}, \mathrm{Po}, \mathrm{Sk}, \mathrm{Su}, \mathrm{Te}, \mathrm{Vn}, \mathrm{Zh}$.

Lophózia ascéndens (Warnst.) Schust. - [CAM], Sk.

Lophozia guttulàta (Lindb \& Arnell) A. Evans (Lophozia longiflora (Nees) Schiffn., L. porphyroleuca (Nees) Schiffn.) - [CAM, MF], If, Lv, Sk, Zh.

Lophozia ventricósa (Dicks.) Dumort. - [CAM, MF, DF], Cv, If, Lv, Sk, Zh.

Lophozia wenzélii (Nees) Steph. (L. confertifolia Schiffn., L. ventricosa var. confertifolia (Schiffn. K.) Müll. - If, Sk.

Lophoziópsis excísa (Dicks.) Konstant. et Vilnet (Lophozia excisa (Dicks.) Dumort.) [CAM, MF, DF, FSZ, SZ, CRM], Ck, If, Ka, Ke, Kr, Ky, Lv, Su, Te, Vn, Zh.

Lophoziopsis longídens (Lindb.) Konstant. et Vilnet (Lophozia longidens (Lindb.) Macoun.). - [CAM, MF, DF], If, Ky, Lv, Sk, Zh.

Lunularia cruciáta (L.) Lindb. - [MF], Ky.

Mánnia androgýna (L.) A. Evans. [FSZ], Ck.

Mannia frágrans (Balbis) Frye \& Clark - [CAM, MF, DF, FSZ, SZ, CRM], Ck. Dn, Do, If, $\mathrm{Ki}, \mathrm{Kr}, \mathrm{Ky}, \mathrm{My}, \mathrm{Sk}, \mathrm{Sp}, \mathrm{Ri}, \mathrm{Vn}, \mathrm{Zh}$.

Mannia pilósa (Homem.) Frye \& Clark - [CAM], Sk.

Mannia triándra (Scop.) Grolle (M. rupestris (Nees) Frye et Clark.) - [CAM], Sk.

Marchantia alpéstris (Nees) Burgeff. (M. polymorpha subsp. montivagans Bischler.\& Boissl.) - [CAM], Cv, Lv, Zk. 
Marchantia polymórpha L. - (subsp. polymorpha and subsp. ruderális Bischler \& Boisselier.) - [CAM, MF, DF, FSZ, SZ, CRM], Ch, Ck, Cv, Dn, Do, If, Ka, Ke, Ki, Km, $\mathrm{Kr}, \mathrm{Ky}, \mathrm{Lu}, \mathrm{Lv}, \mathrm{My}, \mathrm{Od}, \mathrm{Po}, \mathrm{Ri}, \mathrm{Sk}, \mathrm{Sp}, \mathrm{Su}, \mathrm{Te}, \mathrm{Vl}, \mathrm{Vn}, \mathrm{Zh}$.

Marsupélla apiculáta Schiffn. (Gymnomitrion apiculatum (Schiffn.) K. Müll.) - [CAM], Sk.

Marsupella aquática (Schrad.) Schiffn. - [CAM], If.

Marsupella emargináta (Ehrh.) Dumort. - [CAM], If, Sk.

Marsupella fúnckii (F. Weber \& Mohr) Dumort. (Marsupella badensis Schiffn.) [CAM, DF], Cv, If, Lv, Sk.

Marsupella sphaceláta (Gieseke ex Lindenb.) Dumort. - [CAM], If, Sk.

Marsupella sprúcei (Limpr.) H. Bernet. - [CAM], Sk.

Mesoptýchia badénsis (Gottsche ex Rabenhorst.) L. Soderstr. et Váña (Lophozia badensis (Gottsche) Schiffn., Leiocolea badensis (Gott.) Joerg.) - [CAM, MF, SZ], If, Ky, Lv, Sk.

Mesoptýchia bantriénsis (Hook.) L. Soderstr. et Váña (Lophozia bantriensis (Hook.) Steph., L. hornschuchiana (Nees) Mac, Leiocolea bantriensis (Hook.) Joerg., L. mulleri (Nees) Joerg.) - [CAM, CRM], Cv, If, Kr, Lv, Sk.

Mesoptýchia colláris (Nees.) L. Soderstr. et Váña (Lophozia collaris (Nees) Dumort., Jungermannia collaris Nees). - [CAM, DF,CRM], Cv, If, Lv, Sk.

Mesoptýchia heterocólpos (Thed. ex C. Hartm.) L. Soderstr. (Lophozia heterocolpos (Thed. ex C. Hartm.) M. Howe, Leiocolea heterocolpos (Thed.) Buch) - [CAM, MF], Ch, $\mathrm{Cv}, \mathrm{Sk}$.

Metzgéria conjugáta Lindb. - [CAM], If, Lv, Sk.

Metzgeria furcáta (L.) Dumort. - [CAM, MF, DF, FSZ, SZ, CRM], Ch, Ck, Cv, Dn, If, $\mathrm{Ke}, \mathrm{Ki}, \mathrm{Kr}, \mathrm{Ky}, \mathrm{Lv}, \mathrm{Od}, \mathrm{Po}, \mathrm{Sk}, \mathrm{Sp}, \mathrm{Te}, \mathrm{Vn}, \mathrm{Zh}$.

Metzgeria leptonéura Spruce (Metzgeria hamata Lindb.) - [CAM], Sk.

Metzgeria pubéscens (Schrank) Raddi (Apometzgeria pubescens (Schrank) Kuwah.) [CAM,DF], Cv, If, Lv, Sk.

Metzgeria violácea (Ach. ex F.Weber et D. Mohr) Dumort. (Metzgeria fruticulosa (Dicks.) A. Evans, M. furcata var. fruticulosa Lindb.) - [CAM], Cv, Lv, Sk.

Moérckia blýttii (Morch) Brockm. - [CAM], If, Sk.

Mýlia táylori (Hook.) Gray - [CAM, MF], Cv, If, Lv, Sk.

Nárdia geóscyphus (De Not.) Lindb. - [CAM, MF], If, Sk, Zh.

Nardia scaláris Gray - [CAM], If, Sk.

Neoorthocàulis attenuátus (Mart.) L. Soderstr., De Roo et Hedd (Barbilophozia attenuata (Mart.) Loeske, B. gracilis (Schleich.) K. Müll.) - [CAM], If, Lv, Sk.

Neoorthocaulis floérkei (F. Weber \& Mohr) L. Soderstr., De Roo et Hedd (Barbilophozia floerkei (F. Weber \& Mohr) Loeske) - [CAM], If, Sk.

Nowéllia curvifólia (Dicks.) Mitt. - [CAM, MF, DF], Cv, If, Ky, Lv, Sk, Zh.

Odontoschísma denudátum (Mart.) Dumort. - [CAM, MF], If, Zh.

Oxymítra incrassàta (Brot.) Sergio et Sim-Sim (Oxymitra paleacea Bisch. ex Lindenb.) [FSZ, SZ],Ck, Dn, Do, Ki, My, Od.

Pallavicínia lyéllii (Hook.) Carruth. - [CAM], Sk.

Pedinophýllum interrúptum (Nees) Kaal. - [CAM], Sk.

Péllia endiviifólia (Dicks.) Dumort. (P. fabroniana Raddi) - [CAM, MF, DF, FSZ, CRM], Ch, Ck, If, Ka, Km, Kr, Ky, Lv, Po, Sk, Vn, Zh.

Pellia epiphýlla (L.) Corda - [CAM, MF, DF, FSZ, CRM], Ch, If, Ki, Ky, Lv, Po, Sk, Vl, $\mathrm{Zh}$.

Pellia neesiána (Gottsche) Limpr. - [CAM, DF], If, Lv, Sk.

Plagióchila asplenióides (L. emend. Taylor) Dumort. (P. major (Nees) S. Arn.) - [CAM, $\mathrm{MF}], \mathrm{Ch}$, If, Sk. 
Plagiochila porellóides (Torr. ex Nees) Lindenb. (P. asplenioides (L.) Dum. subsp. porelloides (Nees) Schust.) - [CAM, MF, DF, FSZ, CRM], Ck, Cv, If, Km, Kr, Ky, Lv, $\mathrm{Sk}, \mathrm{Te}, \mathrm{Vn}, \mathrm{Zh}$.

Pleurocládula albéscens (Hook.) Grolle (Fuscocephaloziopsis albescens (Hook.) Ván̆a \& L. Söderstr.) - [CAM], Sk.

Porélla arboris-vítae (With) Grolle (P. laevigata (Schrad.) Pfeiff.) - [CAM, CRM], Cv, If, $\mathrm{Kr}, \mathrm{Lv}, \mathrm{Sk}$.

Porella báueri (Schiffn.) C. Jensen - [CAM], Sk.

Porella cordeána (Huebener) Moore - [CAM, CRM], Kr, Sk.

Porella platyphýlla (L.) Pfeiff. - [CAM, MF, DF, FSZ, SZ, CRM], Cv, Ch, Ck, Dn, Do, If, Ke, Ki, Km, Kr, Ky, Lv, Od, Po, Sk, Sp, Te, Vn, Zh.

Préissia quadráta (Scop.) Nees - [CAM, MF, DF,CRM], Cv, If, Ky, Lv, Sk, Zh.

Ptilídium ciliáre (L.) Hampe - [CAM, MF, DF, FSZ, SZ], Ch, If, Ky, Po, Sk, Su, Zh.

Ptilidium pulchérrimum (Weber) Vainio - [CAM, MF, FSZ, SZ, CRM], Cv, Ch, Ck, Dn, If, Ka, Km, Kr, Ky, Lv, Po, Sk, Sp, Su, Te, Vn, Vl, Zh.

Rádula complanáta (L.) Dumort. - [CAM, MF, DF, FSZ, SZ, CRM], Cv, Ch, Ck, Dn, Do, If, Ka, Ke, Ki, Km, Kr, Ky, Lv, Lu, My, Od, Po, Sk, Sp, Su, Te, Vl, Vn, Zh.

Radula lindbergíana Gottsche ex C. Hartm. - [CAM, MF] Cv, If, Sk, Zh.

Rebóulia hemisphaérica (L.) Raddi - [CAM, MF, DF, FSZ, SZ, CRM], Ck, Dn, Do, Ki, Kr, $\mathrm{K}$ y, Lv, Sk, Sp, Vn, Zh.

Riccárdia chamaédryfolia (With.) Grolle ( $R$. sinuata (Dicks.)Trev., Aneura major $K$. Müll.) - [CAM, CRM], If, Kr, Lv, Sk.

Riccardia incurváta Lindb. (Aneura incurvata Steph.) - [MF, DF], Ky, Lv, Ri.

Riccardia latífrons (Lindb.) Lindb. (Aneura latifrons Lindb.) - [CAM, MF, DF], Ch If, Ky, Lv. Ri, Sk, Zh.

Riccardia multífida (L.) S.Gray (Aneura multifida Dum.) - [CAM, MF, DF], Cv, If, Ky, Lv, Sk, Zh.

Riccardia palmáta (Hedw.) Carruth. (Aneura palmata Dum.) - [CAM, MF, DF], Ch, Cv, If, Lv, Sk, Zh.

Ríccia beyrichiána Hampe ex Lehm. - [MF, SZ], Dn, Ky.

Riccia bifúrca Hoffm. - [MF, FSZ], Ch, Ky, Lv.

Riccia canaliculáta Hoffm. - Ch, Ck, Dn, Ky.

Riccia cavernósa Hoffm. - [MF, DF, FSZ, SZ], Ch, Ck, Dn, Ky, Sp, Po.

Riccia ciliáta Hoffm. - [CAM, MF, DF, FSZ, SZ, CRM], Dn, Do, Ke, Ki, Kr, Ky, Lu, $\mathrm{My}, \mathrm{Sk}, \mathrm{Zh}$.

Riccia ciliífera Link ex Lindenb. - [MF, FSZ, SZ], Ck, Dn, Do, Ke, Ky, Mk, Sp.

Riccia criníta Hoffm. (R. trichocarpa M.Howe, R. canescens Steph.) - [CAM, MF, SZ], $\mathrm{Ke}, \mathrm{Ky}, \mathrm{Sk}, \mathrm{Zh}$.

Riccia crystallína L. emend. Raddi - [CAM, MF, DF, FSZ, SZ], Ch, Ck, Dn, If, Ki, Ky, Po, Sp.

Riccia erinácea Schiffn. (Riccia gougetiana var. armatissima Levier ex Müll. Frib, $R$. gougetiána var. erinacea Schiffn.) - [SZ], My.

Riccia flúitans L. emend. Lorbeer - [CAM, MF, DF, FSZ, SZ], Ch, Ck, Dn, Ka, Km, $\mathrm{Ky}, \mathrm{Lv}, \mathrm{Sk}, \mathrm{Su}, \mathrm{Te}, \mathrm{Vl}$.

Riccia fróstii Austin - [MF, FSZ,SZ], Ck, Ky, Po, Su.

Riccia gláuca L. - [CAM, MF, DF, FSZ, SZ] Ch, Ck, Dn, If, Ka, Ki, Ky, Lv, My, Po, Sk, $\mathrm{Sp}, \mathrm{Vn}, \mathrm{Zh}$.

Riccia gougetiána Durieu \& Mont. - [SZ], Dn, Sp.

Riccia huebeneriána Lindenb. - [MF], Ch, Ky, Zh.

Riccia lamellósa Raddi - [SZ], Dn, Ka, Ke. 
Riccia papillósa Moris (R. pseudopapillosa Levier ex Steph.) - [FSZ,SZ], Ck, Dn, Do, $\mathrm{Ke}, \mathrm{Ki}, \mathrm{Lu}, \mathrm{My}$.

Riccia rhenána Lorbeer - [MF, FSZ,SZ], Ck, Dn, Ky.

Riccia rhenána Lorbeer var. violácea M.F.Boiko [SZ], Dn.

Riccia sorocárpa Bisch. - [CAM, MF, DF, FSZ, SZ, CRM], Ch, Ck, If, Ka, Ki, Km, Kr, Ky, Zh.

Ricciocárpos nátans (L.) Corda - [CAM, MF, DF, FSZ, SZ], Ch, Dn, Ka. Ky, Sk, Lv, Te, Vl, Zh.

Sautéria alpína (Nees) Nees - [CAM], Sk.

Scapánia aequilóba (Schwaegr.) Dumort. - [CAM], If, Lv, Sk.

Scapania apiculáta Spruce - [CAM], Lv, Sk.

Scapania áspera M. Bernet. \& H. Bernet. - [CRM], Kr.

Scapania calcícola (H. Arn. \& J. Perss.) Ingham. - [CRM], Kr.

Scapania compácta (Roth) Dumort. - [CRM], Kr.

Scapania cúrta (Mart.) Dumort. - [CAM, MF, DF, FSZ], Ch, Ck, If, Lv, Sk, Te, Zt.

Scapania cuspidulígera (Nees) Müll. Frib. [CAM], If, Sk,

Scapania helvética Gottsche - [CAM], If, Sk.

Scapania irrígua (Nees) Nees - [CAM, MF], Cv, If, Ky, Lv, Sk, Te, Zt.

Scapania mucronáta $\mathrm{H}$. Buch - $[\mathrm{CAM}]$, Sk.

Scapania nemórea (L.) Grolle (S. nemorosa (L.) Dum.) - [CAM, MF, DF, FSZ], Cv, If, $\mathrm{Ka}, \mathrm{Lv}, \mathrm{Sk}, \mathrm{Zt}$.

Scapania paludícola Loeske \& Müll. Frib. - [MF], Zt.

Scapania parvifólia Warnst. - [CAM], If, Sk.

Scapania subalpína (Nees ex Lindenb.) Dumort. - [CAM], If.

Scapania uliginósa (Sw. ex Lindenb.) Dumort. - [CAM], If, Sk.

Scapania umbrósa (Schrad.) Dumort. - [CAM], [CAM], If, Lv, Sk.

Scapania unduláta (L.) Dumort. - [CAM, MF], If, Lv, Sk, Zt.

Scapania verrucósa Heeg - [CAM], Cv, If, Lv, Sk.

Schistochilópsis incisa (Schrad.) Konstant. 1994 (Lophozia incisa (Schrad.) Dumort.) [CAM, MF, DF], If, Lv, Sk, Zt.

Schljacoviànthus quadrílobus (Lindb.) 2010 (Barbilophozia quadriloba (Lindb.) Loeske) - [CAM], If.

Solenostóma confertíssimum (Nees) Schljakov) 1980 (Jungermannia confertissima Nees, Solenostoma levieri (Steph. ) - [CAM], If, Sk.

Solenostoma gracíllimum (Sm.) R.M. Schust. (Plectocolea crenulata Evans, Jungermannia gracillima Sm., Solenostoma crenulatum (Sm.) Mitt.) - [CAM, DF], Cv, If, Lv, Sk.

Solenostoma hyálinum (Lyell ex Hook.) Mitt. (Plectocolea hyalina (Lyell) Mitt. (Jungermannia hyalina Lyell) - [CAM, MF, DF, FSZ], Ch, Ck, Cv, If, Km, Lv, Sk, Te, $\mathrm{Zh}$.

Solenostoma obovátum (Nees) C. Massal. 1903 (Plectocolea obovata (Nees) Mitt., Jungermannia obovata Nees) - [CAM], If, Sk.

Solenostoma sphaerocárpum (Hook.) Steph. (Jungermannia sphaerocarpa Hook.) [CAM], Cv, If, Lv, Sk.

Sphenolobus minútus (Schreb.) Berggr. (Anastrophyllum minutum (Schreb.) R.M. Schust.) - [CAM], If, Sk.

Syzygiélla autumnális (DC.) K. Feldberg, Váña, Hentschel et Heinrichs 2010 (Jamesoniella autumnalis (DC.) Steph.) - [CAM, MF], If, Ky, Lv, Sk, Zh.

Targiónia hypophýlla L. - [CRM], Kr.

Trichocólea tomentélla (Ehrh.) Dumort. - [CAM, MF, DF, FSZ], Ch, Cv, If, Ka, Lv, Sk, Te, Zh. 
Trilophózia quinquedentáta (Huds.) Bakalin (Tritomaria quinquedentata (Huds.) H. Buch, Barbilophozia quinquedentata Loeske) - [CAM], Cv, If, Sk.

Tritomária exsécta (Schrad.) Loeske - [CAM], Cv, If, Lv, Sk.

Tritomaria exsectifórmis (Breidl.) Loeske - [CAM], If, Sk, Zh.

Tritomaria scítula (Taylor) Jörg. - [CAM], Sk.

\section{BRYOPHYTA}

Abietinélla abietína (Hedw.) Fleisch. (Thuidium abietinum (Hedw.) Schimp.) - [CAM, MF, DF, FSZ, SZ, CRM], Ch, Ck, Cv, Dn, Do, If, Ka, Ki, Km, Kr, Ky, Lu, Lv, My, Od, Po, Ri, Sk, Sp, Su, Te, Vn, Vl, Zh.

Acáulon múticum (Hedw.) H. Müll. (A. rubrum (Röhl) Grout) - [MF, DF, FSZ, SZ], Dn, Ky, Lv, Po, Vn, Zh.

Acaulon triquétrum (Spruce) H. Müll. - [MF, FSZ, CRM], Ck, Kr, Ky, Vn.

Alleniélla besseri (Lob.) S.Olsson, Enroth \& D.Quandt (Neckera besseri (Lob.) Jur., Homalia besseri Lobarz.) - [CAM, MF, DF, FSZ, SZ, CRM], Ck, Cv, Dn, Do, If, Ke, $\mathrm{Km}, \mathrm{Kr}, \mathrm{Ky}, \mathrm{Lu}, \mathrm{Lv}, \mathrm{My}, \mathrm{Ri}, \mathrm{Sk}, \mathrm{Te}, \mathrm{Vn}, \mathrm{Zh}$.

Alleniella complanáta S.Olsson, Enroth \& D.Quandt (Neckera complanata (Hedw.) Huebener, Homalia complanata (Hedw.) De Not.) - [CAM, MF, DF, FSZ, SZ, CRM], $\mathrm{Ch}, \mathrm{Ck}, \mathrm{Cv}$, Dn, If, Kr, Ky, Lv, My, Ri, Sk, Su, Te, Vl, Vn, Zh.

Aloína aloídes (W. Koch ex Schultz) Kindb. - [DF, FSZ], Ky, Te.

Aloina ambígua (Bruch \& Schimp.) Limpr. - [CAM, DF, FSZ, SZ], Ck, If, Lv, My, Po, $\mathrm{Ri}, \mathrm{Te}$.

Aloina breviróstris (Hook. \& Grev.) Kindb. - [MF, DF], Su, Te.

Aloina rígida (Hedw.) Limpr. - [CAM, MF, DF, FSZ, SZ], Ch, Ck, Cv, Do, Ka, If, Kr, $\mathrm{Ky}, \mathrm{Lv}, \mathrm{Po}, \mathrm{Ri}, \mathrm{Sk}, \mathrm{Su}, \mathrm{Te}, \mathrm{Vl}$, .Vn, Zh

Amblýodon dealbátus (Hedw.) P.Beauv. - [DF], Lv.

Amblystégium juratzkánum Schimp. - [CAM, MF, DF, FSZ, SZ, CRM], Ch, Dn, If, $\mathrm{Ka}, \mathrm{Ke}, \mathrm{Kr}, \mathrm{Ky}, \mathrm{Lu}, \mathrm{Lv}, \mathrm{My}, \mathrm{Po}, \mathrm{Ri}, \mathrm{Sk}, \mathrm{Su}, \mathrm{Vn}, \mathrm{Vl}$.

Amblystegium sérpens (Hedw.) Schimp. - [CAM, MF, DF, FSZ, SZ, CRM], Ch, Ck, $\mathrm{Cv}, \mathrm{Dn}, \mathrm{Do}, \mathrm{If}, \mathrm{Ka}, \mathrm{Ke}, \mathrm{Ki}, \mathrm{Km}, \mathrm{Kr}, \mathrm{Ky}, \mathrm{Lu}, \mathrm{Lv}, \mathrm{My}, \mathrm{Od}, \mathrm{Po}, \mathrm{Ri}, \mathrm{Sk}, \mathrm{Sp}, \mathrm{Su}, \mathrm{Te}, \mathrm{Vn}, \mathrm{Vl}$, $\mathrm{Zh}$.

Amphídium lappónicum (Hedw.) Schimp. - [CAM], If, Sk.

Amphidium mougeótii (Schimp.) Schimp. - [CAM, CRM], If, Kr, Sk.

Anacámptodon splachnóides (Froel. ex Brid.) Brid. - [CAM], Cv, If, Sk.

Andreaéa alpéstris (Thed.) Schimp. - [CAM], If, Sk.

Andreaea rupéstris Hedw.- [CAM], If, Lv, Sk.

Anoectángium aestívum (Hedw.) Mitt. - [CAM], Sk.

Anoectangium handélii Schiffn. - [CAM], Kr.

Anomóbryum juláceum (Schrad. ex P.Gaertn. et al.) Schimp. (Bryum julaceum Schrad. ex P.Gaertn. et al.) - [CAM], If.

Anómodon attenuátus (Hedw.) Huebener - [CAM, MF, DF, FSZ, SZ, CRM], Ch, Ck, Cv, Dn, Do, If, Ka, Ki, Km, Kr, Ky, Lu, Lv, Od, Po, Ri, Sk, Su, Te, Vn, Vl, Zh.

Anomodon longifólius (Schleich. ex Brid.) C. Hartm.- [CAM, MF, DF, FSZ, SZ, CRM], Ch, Ck, Cv, Dn, Do, If, Ka, Ki, Km, Kr, Ky, Lu, Lv, Od, Po, Ri, Sk, Su, Te, Vn, Zh.

Anomodon rostrátus (Hedw.) Schimp. - [CAM, DF], Lv, Sk.

Anomodon rúgelii (H. Müll.) Keissl. (A. apiculatus Sull.) - [CAM, DF], Cv, If, Km, Sk. Anomodon viticulósus (Hedw.) Hook. \& Taylor) - [CAM, MF, DF, FSZ, SZ, CRM], $\mathrm{Ch}, \mathrm{Ck}, \mathrm{Cv}$, Dn, Do, If, Ka, Ke, Ki, Km, Kr, Ky, Lu, Lv, My, Od, Po, Ri, Sk, Sp, Su, Te, Vl, Vn, Zh.

Antitríchia curtipéndula (Hedw.) Brid. - [CAM, DF, FSZ, CRM], If, Kr, Lv, Sk, Zh. Arctóa fulvélla (Dicks.) Bruch \& Schimp. (Dicranum fulvellum Smith.) - [CAM], Sk. 
Átrichum angustátum (Brid.) Bruch \& Schimp. - [CAM, SZ], If, Lu, Lv, Sk.

Atrichum críspulum Schimp. ex Besch. (Atrichum undulatum var. minor (Hedw.) Web. et Mohr - [CAM, DF, CRM], If, Kr, Lv.

Atrichum flavisétum Mitt. (A. undulatum var. haussknechtii Frye) - [CAM, DF, CRM], If, $\mathrm{Kr}, \mathrm{Lv}, \mathrm{Sk}$.

Atrichum tenéllum (Röhl.) Bruch \& Schimp. - [CAM, MF, DF, FSZ, SZ], Ch, Cv, If, $\mathrm{Km}, \mathrm{Ky}, \mathrm{Lu}, \mathrm{Lv}, \mathrm{Po}, \mathrm{Ri}, \mathrm{Sk}, \mathrm{Su}, \mathrm{Zh}$.

Atrichum undulátum (Hedw.) P. Beauv. (Polytrichum undulatum Hedw.) - [CAM, MF, DF, FSZ, SZ, CRM], Ch, Ck, Cv, Dn, If, Ka, Ki, Km, Kr, Ky, Lu, Lv, Po, Ri, Sk, Sp, Su, Te, Vn, Vl, Zh.

Aulacómnium andrógynum (Hedw.) Schwaegr. - [CAM, MF, DF, SZ, CRM], Ch, If, $\mathrm{Ke}, \mathrm{Kr}, \mathrm{Lv}, \mathrm{Lu}, \mathrm{Sk}, \mathrm{Zh}$.

Aulacomnium arenopaludósum Boiko - [SZ], Ke, My.

Aulacomnium palústre (Hedw.) Schwaegr. - [CAM, MF, DF, FSZ, SZ, CRM], Ch, Ck, $\mathrm{Cv}$, Dn, Do, If, Ka, Ke, Ki, Km, Ky, Lu, Lv, My, Po, Ri, Sk, Su, Te, Vl, Vn, Zh.

Bárbula convolúta Hedw. - [CAM, MF, DF, FSZ, SZ, CRM], Ch, Ck, Dn, If, Ka, Km, $\mathrm{Kr}, \mathrm{Ky}, \mathrm{Lv}, \mathrm{Od}, \mathrm{Po}, \mathrm{Ri}, \mathrm{Sk}, \mathrm{Su}, \mathrm{Te}, \mathrm{Vl}, \mathrm{Vn}$.

Barbula crócea (Brid.) F. Weber \& Mohr - [CAM], Sk.

Barbula enderésii Garov. - [DF], Te

Barbula unguiculáta Hedw. - [CAM, MF, DF, FSZ, SZ, CRM], Ch, Ck, Cv, Dn, Do, If, $\mathrm{Ka}, \mathrm{Ke}, \mathrm{Ki}, \mathrm{Km}, \mathrm{Kr}, \mathrm{Ky}, \mathrm{Lu}, \mathrm{Lv}, \mathrm{My}, \mathrm{Od}, \mathrm{Po}, \mathrm{Ri}, \mathrm{Sk}, \mathrm{Sp}, \mathrm{Su}, \mathrm{Te}, \mathrm{Vn}, \mathrm{Vl}, \mathrm{Zh}$.

Bartrámia halleriána Hedw. - [CAM, CRM], Cv, If, Kr, Sk.

Bartramia ithyphýlla Brid. - [CAM, MF, DF, SZ], If, Ri, Sk, Sp, Te, Zh.

Bartramia pomifórmis Hedw. - [CAM, MF, DF, FSZ, CRM], Cv, If, Kr, Lv, Ri, Sk, Te, Vn, Zh.

Blíndia acúta (Hedw.) Bruch \& Schimp. - [CAM], Cv, If, Lv, Sk.

Brachydóntium trichódes (F.Weber) Milde (Gymnostomum trichodes F.Weber) [CAM], Cv, If, Lv, Sk.

Brachytheciástrum trachypódium (Brid.) Ignatov \& Huttunen (Brachythecium trachypodium (Brid.) Schimp.) - [CAM], Lv.

Brachytheciastrum velútinum (Hedw.) Ignatov \& Huttunen (Brachythecium velutinum (Hedw.) Schimp.) - [CAM, MF, DF, FSZ, SZ, CRM], Ch, Ck, Cv, Dn, Do, If, Ka, Ke, Ki, $\mathrm{Km}, \mathrm{Kr}, \mathrm{Ky}, \mathrm{Lu}, \mathrm{Lv}, \mathrm{My}$, Od, Po, Ri, Sk, Sp, Su, Te, Vn, Vl, Zh.

Brachythécium álbicans (Hedw.) Schimp. - [CAM, MF, DF, FSZ, SZ, CRM], Ch, Ck, $\mathrm{Cv}$, Dn, Do, If, Ka, Ke, Ki, Km, Kr, Ky, Lu, Lv, My, Od, Po, Ri, Sk, Su, Te, Vn, Vl, Zh.

Brachythecium campéstre (H. Müll.) Schimp. - [CAM, MF, DF, FSZ, SZ, CRM], Ch, $\mathrm{Ck}, \mathrm{Cv}$, Dn, Do, If, Ka, Ke, Ki, Km, Kr, Ky, Lu, Lv, My, Po, Ri, Sk, Su, Te, Vn, Zh.

Brachythecium cirrósum (Schwaegr.) Schimp. (Cirriphyllum cirrosum (Schwägr.) Grout) - [CAM], If, Sk.

Brachythecium geheébii Milde (Homalothecium geheebii (Milde) Wigh) - [CAM, MF, DF, FSZ, SZ, CRM], If, Lv, Sk.

Brachythecium glareósum (Bruch ex Spruce) Schimp.) - [CAM, MF, DF, FSZ, SZ, CRM], - Ch, Ck, Cv. Dn, Do, If, Km, Kr, Lu, Lv, My, Po, Ri, Sk, Sp, Te, Vn, Zh.

Brachythecium laétum (Brid.) Schimp. - [CAM], If, Sk.

Brachythecium mildeánum (Schimp.) Schimp.) - [CAM, MF, DF, FSZ, SZ, CRM], $\mathrm{Ch}, \mathrm{Ck}, \mathrm{Cv}$, Dn, Do, If, Ka, Ke, Ki, Km, Kr, Ky, Lu, Lv, My, Od, Po, Ri, Sk, Su, Te, Vn, $\mathrm{Vl}, \mathrm{Zh}$.

Brachythecium rivuláre Schimp. - [CAM, MF, DF, FSZ, SZ, CRM], Ch, Ck, Cv, Dn, If, $\mathrm{Ke}, \mathrm{Ki}, \mathrm{Km}, \mathrm{Kr}, \mathrm{Ky}, \mathrm{Lv}, \mathrm{My}, \mathrm{Po}, \mathrm{Ri}, \mathrm{Sk}, \mathrm{Sp}, \mathrm{Su}, \mathrm{Te}, \mathrm{Vl}, \mathrm{Vn}, \mathrm{Zh}$. 
Brachythecium rutábulum (Hedw.) Schimp. - [CAM, MF, DF, FSZ, SZ, CRM], Ch, $\mathrm{Ck}, \mathrm{Cv}$, Dn, Do, If, Ka, Ke, Ki, Km, Kr, Ky, Lu, Lv, My, Od, Po, Ri, Sk, Sp, Su, Te, Vl, $\mathrm{Vn}, \mathrm{Zh}$.

Brachythecium salebrósum (Hoffm. ex F. Weber \& Mohr) Schimp. - [CAM, MF, DF, FSZ, SZ, CRM], Ch, Ck, Cv, Dn, Do, If, Ka, Ke, Ki, Km, Kr, Ky, Lu, Lv, My, Od, Po, Ri, $\mathrm{Sk}, \mathrm{Su}, \mathrm{Te}, \mathrm{Vn}, \mathrm{Vl}, \mathrm{Zh}$.

Brachythecium tommasínii (Sendt. ex Boulay) Ignatov \& Huttunen (Cirriphyllum tommasinii (Sendtn. ex Boulay) Grout, C. vaucheri (Schimp.) Loeske \& Fleisch) - [CAM, DF, CRM], Km, Kr, Lv, Sk, Te.

Breidléria praténsis (W. Koch ex Spruce) Loeske (Hypnum pratense W.D.J. Koch ex Spruce) - [CAM, MF], If, Ky Sk.

Bryoerythrophýllum alpigénum (Vent.) Chen (B. recurvirostrum var. dentatum (Schimp.) H.A.Crum, Steere \& L.E.Anderson, Didymodon alpigenum Vent.) - [CAM], Sk.

Bryoerythrophyllum recurviróstrum (Hedw.) Chen (Erythrophyllum recurvirostrum (Hedw.) Lazar.) - [CAM, MF, DF, FSZ, SZ, CRM], Ch, Ck, Cv, Dn, Do, If, Ka, Ki, Km, $\mathrm{Kr}, \mathrm{Ky}, \mathrm{Lv}, \mathrm{My}, \mathrm{Po}, \mathrm{Sk}, \mathrm{Su}, \mathrm{Te}, \mathrm{Vn}, \mathrm{Zh}$.

Brýum argénteum Hedw. - [CAM, MF, DF, FSZ, SZ, CRM], Ch, Ck, Cv, Dn, Do, If, $\mathrm{Ka}, \mathrm{Ke}, \mathrm{Ki}, \mathrm{Km}, \mathrm{Kr}, \mathrm{Ky}, \mathrm{Lu}, \mathrm{Lv}, \mathrm{My}, \mathrm{Od}, \mathrm{Po}, \mathrm{Ri}, \mathrm{Sk}, \mathrm{Sp}, \mathrm{Su}, \mathrm{Te}, \mathrm{Vn}, \mathrm{Vl}, \mathrm{Zh}$.

Bryum bádium (Brid.) Schimp. - [CAM, MF, DF, SZ, CRM], Cv, If, Ka, Ke, Ki, Kr, Lu, Lv, My, Od, Ri, Sk, Te.

Bryum caespitícium Hedw. - [CAM, MF, DF, FSZ, SZ, CRM], Ch, Ck, Cv, Dn, Do, If, $\mathrm{Ka}, \mathrm{Ke}, \mathrm{Ki}, \mathrm{Km}, \mathrm{Kr}, \mathrm{Ky}, \mathrm{Lu}, \mathrm{Lv}, \mathrm{My}, \mathrm{Od}, \mathrm{Po}, \mathrm{Ri}, \mathrm{Sk}, \mathrm{Sp}, \mathrm{Su}, \mathrm{Te}, \mathrm{Vn}, \mathrm{Vl}, \mathrm{Zh}$.

Bryum dichotómum Hedw. - [CAM, MF, SZ, CRM], Cv, If, Ka, Ke, Kr,Lv, Sk.

Bryum élegans Nees (B. capillare var. elegans (Nees) Husn) - [CAM, MF, SZ, CRM], $\mathrm{Cv}$, If, $\mathrm{Kr}, \mathrm{Lv}, \mathrm{Mk}, \mathrm{Sk}$, Su.

Bryum fúnckii Schwaegr. - [CAM, DF, FSZ, SZ, CRM], Cv, If, Ka, Ke, Kr, Lv, Mk, Sk, Su.

Bryum gemmipárum De Not. (B. alpinum subsp. gemmiparum (De Not.) Kindb.) [CAM, CRM], Kr, Sk.

Bryum intermédium (Brid.) Blandow - [CAM, MF, DF, SZ], Do, Ky, Lv, Sk, Te.

Bryum klínggraéffii Schimp. - [CAM, MF, DF, FSZ, SZ, CRM], Ch, Ck, Cv, Do, Ka, $\mathrm{Ki}, \mathrm{Km}, \mathrm{Ky}, \mathrm{Lv}, \mathrm{Ri}, \mathrm{Su}, \mathrm{Vn}$.

Bryum knówltonii Barnes (B. lacustre (F.Weber \& D.Mohr) Blandow non Brid.) [CAM], Sk.

Bryum kúnzei Hornsch. - [CAM, DF, FSZ, SZ], If, Ka, Kr, Ri, Sk, Vn.

Bryum palléscens Schleich. ex Schwaegr. - [CAM, MF, DF, FSZ, SZ, CRM], Ch, Ck, Cv. If, Ka, Ke, Ki, Km, Kr, Ky, Lu, Lv, My, Po, Ri, Sk, Sp, Su, Te, Vn.

Bryum ruderále Crundw. \& Nyholm - [MF, FSZ, SZ], Do, Ky, Kr, Sp.

Bryum schléicheri DC. - [CAM], If, Sk.

Bryum subapiculátum Hampe - [MF, FSZ, SZ], Ka, Ke, Kr. Ky, Lv,Vn.

Bryum tenuisétum Limpr. - $[\mathrm{MF}], \mathrm{Ka}, \mathrm{Ky}$.

Bryum turbinátum (Hedw.) Turn. - [CAM, DF, SZ, CRM], Cv, Do, Kr, Lv, My, Sk, Te. Bryum veronénse De Not. (Bryum argenteum subsp. veronense (De Not.) J.J. Amann) [CAM], Sk.

Bryum violáceum Crundw. \& Nyh. - [MF, DF, FSZ, SZ], Ch, Cv, Do, Ka, Ki, Ky, Lu, Lv, Sp.

Bryum wéigélii Spreng. - [CAM, MF, SZ], Ch, Do, Km, Sk.

Buxbáumia aphýlla Hedw. - [CAM, MF, DF, FSZ], Ch, Cv, If, Ka, Ky, Lv, Po, Ri, Sk, $\mathrm{Su}, \mathrm{Te}, \mathrm{Vl}, \mathrm{Zh}$. 
Buxbaumia víridis (Moug. ex Lam. \& DC.) Brid. ex Moug. \& Nestl. - [CAM, DF, CRM], Cv, If, Kr, Lv, Sk.

Callicládium haldaniánum (Grev.) Crum (Heterophyllium haldanianum (Grev.) Fleish.). - [CAM, MF, DF, FSZ, SZ], Ch, Ck, Dn, Do, If, Ka, Ki, Km, Ky, Lu, Lv, Od, Po, Ri, Sk, $\mathrm{Sp}, \mathrm{Su}, \mathrm{Te}, \mathrm{Vl}, \mathrm{Vn}, \mathrm{Zh}$.

Calliérgon cordifólium (Hedw.) Kindb. - [CAM, MF, DF, FSZ, SZ], Ch, Ck, Cv, Dn, Do, If, Ka, Ke, Ki, Ky, Lu, Lv, Po, Ri, Sk, Su, Te, Vl, Vn, Zh.

Calliergon gigánteum (Schimp.) Kindb. - [CAM, MF, DF, FSZ], Ch, Ck, If, Ka, Ky, Lv, Po, Ri, Sk, Su, Te, Vl, Vn, Zh.

Calliergon ríchardsonii (Mitt.) Kindb. - [CAM], If.

Calliergonélla cuspidáta (Hedw.) Loeske) - [CAM, MF, DF, FSZ, SZ, CRM], Ch, Ck, $\mathrm{Cv}$, Dn, Do, If, Ka, Ke, Ki, Km, Kr, Ky, Lu, Lv, Po, Ri, Sk, Su, Te, Vl, Vn, Zh.

Calliergonella líndbergii (Mitt.) Hedenäs (Hypnum lindbergii Mitt., Breidleria arcuata (Lindb.) Loeske) ) - [CAM, MF, DF, FSZ], Cv, If, Ky, Lv, Ri, Sk, Su, Te, Vl, Zh.

Campyliadélphus chrysophýllus (Brid.) R.S.Chopra (Campylium chrysophyllum (Brid.) Lange) - [CAM, MF, DF, FSZ, SZ, CRM], Ch, Cv, Do, If, Ki, Kr, Lv, Ri, Sk, Te, Km.

Campyliadelphus elódes (Lindb.) Kanda (Campylium elodes (Lindb.) Kindb., Chrysohypnum helodes (Spruce) Loeske) - [CAM, MF], If, V1.

Campylídium calcáreum (Crundw. \& Nyholm) Ochyra (Campylophyllum calcareum (Crundw. \& Nyholm) Hedenäs, Campylium calcareum Crundw. \& Nyholm) - [DF, SZ, $\mathrm{CRM}$, Do, Ka, Km, Kr, My, Sp.

Campýlium proténsum (Brid.) Kindb. - [CAM, MF, CRM], Km, Kr, Ri, Sk, Vl.

Campylium sommerféltii (Myrin) Lange, (Campylophyllum sommerfeltii (Myrin) Hedenäs, Chrysohypnum sommerfeltii (Myrin) Roth) - [CAM, MF, DF, FSZ, SZ, CRM], $\mathrm{Ch}, \mathrm{Ck}, \mathrm{Cv}, \mathrm{Dn}, \mathrm{Do}$, If, Ka, Ki, Km, Kr, Ky, Lu, Lv, My, Po, Ri, Sk, Sp, Su, Te, Vl, Vn, Zh.

Campylium stellátum (Hedw.) Lange \& C. Jensen (Campyliadelphus stellatus (Hedw.) Kanda) - [CAM, MF, DF, FSZ, SZ, CRM], Cv, If, Km, Kr, Ky, Lv, Ri, Sk, Te, Vl, Zh.

Campylophýllum halléri (Hedw.) Fleisch. (Campylium halleri (Hedw.) Lindb.) [CAM], If, Sk.

Campylópus frágilis (Brid.) Bruch \& Schimp. - [CAM, MF], If, Lv, Ky, Sk.

Campylopus grácilis (Mitt.) Jaeg. (C. schwarzii Schimp.) - [CAM], Cv, Sk.

Campylopus introfléxus (Hedw.) Brid. - [DF], Lv.

Campilopus pyrifórmis (Schultz) Brid. (C. fragilis var. pyriformis (Schultz) Agst.) [CAM], Sk.

Campylostélium saxícola (F.Weber \& Mohr) Bruch \& Schimp. (Campylopus saxicola (F. Weber \& D.Mohr) Bruch \& Schimp.) - [CAM], Cv, If, Lv.

Campylostelium stríctum Solms - [CAM], Cv.

Ceratodon purpúreus (Hedw.) Brid. - [CAM, MF, DF, FSZ, SZ, CRM], Ch, Ck, Cv, Dn, Do, If, Ka, Ke, Ki, Km, Kr, Ky, Lu, Lv, My, Od, Po, Ri, Sk, Sp, Su, Te, Vn, Vl, Zh.

Cinclídium stýgium Sw. - [CAM], Cv, Sk.

Cinclidótus aquáticus (Hedw.) Bruch \& Schimp. - [CRM], Kr.

Cinclidotus fontinalóides (Hedw.) P. Beauv. - [CAM, MF, CRM], Kr, My, Ri, Sk, Zh.

Cinclidotus ripárius (Host ex Brid.) Arnott (C. nigricans (Brid.) Wijk \& Marg.) [CRM], Kr.

Cirriphýllum crassinérvium (Taylor) Loeske \& Fleisch. (Eurhynchium crassinervium (Taylor) Schimp.) - [CAM, MF, DF, SZ, CRM], Do, If, Km, Kr, Lv, Mk, Ri, Sk, Te.

Cirriphyllum pilíferum (Hedw.) Grout - [CAM, MF, DF, FSZ], Ch, Cv, If, Km, Ky, Lv, Po, Ri, Sk, Te, Vn, Zh.

Cleistocarpídium palústre (Bruch \& Schimp.) Ochyra \& Bednarek - Ochyra (Pleuridium palustre (Bruch \& Schimp.) Bruch \& Schimp.) - [CAM, FSZ], Ck, If. 
Climácium dendróides (Hedw.) F.Weber \& Mohr) - [CAM, MF, DF, FSZ, SZ, CRM], $\mathrm{Ch}, \mathrm{Ck}, \mathrm{Cv}, \mathrm{Dn}, \mathrm{Do}$, If, Ka, Ki, Km, Kr, Ky, Lu, Lv, Po, Ri, Sk, Sp, Su, Te, Vl, Vn, Zh.

Cnéstrum schísti (F.Weber \& Mohr) Hag. (Cynodontium schistii Lindb.) - [CAM], Sk.

Conárdia compácta (Drumm. ex Müll. Hal.) H. Robins. (Amblystegium compactum (Drumm. ex Müll.Hal.) Austin) - [DF, FSZ], Ck, Cv.

Coscínodon cribrósus (Hedw.) Spruce - [CAM, CRM], Cv, If, Kr.

Cratonéuron curvicáule (Jur.) Roth (Cratoneuron filicinum var. curvicaule (Jur.) Mönk.) - [CAM], If, Sk.

Cratoneuron filicínum (Hedw.) Spruce) - [CAM, MF, DF, FSZ, SZ, CRM], Ch, Ck, Cv, If, Ka, Km, Kr, Ky, Lv, My, Ri, Sk, Su, Te, Vl, Vn, Zh.

Crossídium squamíferum (Viv.) Jur. - [SZ, CRM], Kr.

Ctenídium mollúscum (Hedw.) Mitt. - [CAM, SZ, CRM], Cv, If, Kr, Lv, Sk.

Cynodóntium brúntonii (Sm.) Bruch \& Schimp. - [CAM, CRM], Kr, Sk.

Cynodontium graciléscens (F.Weber \& Mohr) Schimp. (Oncophorus gracilescens Lindb.) - [CAM], Cv, If.

Cynodontium polycárpon (Hedw.) Schimp. (Oncophorus polycarpus Brid.) - [CAM, MF, DF, FSZ], Cv, If, Ky, Sk, Te, Vn.

Cynodontium strumíferum (Hedw.) Lindb. (Oncophorus strumifer Brid.) - [CAM, MF], $\mathrm{Cv}$, If, Lv, Sk.

Cynodontium tenéllum (Schimp.) Limpr. - [CAM], Sk.

Dichelýma capillaceum (Dicks.) Myrin - [MF], Zh.

Dichelyma falcátum (Hedw.) Myrin - [CAM], Sk.

Dichodóntium flavéscens (Dicks.) Lindb.- [CAM], Sk.

Dichodontium palústre (Dicks.) M. Stech (Dicranella palustris (Dicks.) Crundw.) [CAM], If, Sk.

Dichodontium pellúcidum (Hedw.) Schimp. - [CAM, FSZ], Cv, If, Lv, Sk, Vn, Zh.

Dicranélla cerviculáta (Hedw.) Schimp. - [CAM, MF, DF, FSZ], Ch, Ck, Cv, If, Ka, $\mathrm{Ky}, \mathrm{Lv}, \mathrm{Po}, \mathrm{Ri}, \mathrm{Sk}, \mathrm{Su}, \mathrm{Vn}, \mathrm{Vl}, \mathrm{Zh}$.

Dicranella críspa (Hedw.) Schimp. - [CAM, MF, DF], Ch, If, Lv, Zt.

Dicranella heteromálla (Hedw.) Schimp. - [CAM, MF, DF, FSZ, SZ], Ch, Ck, Cv, Do, If, Ki, Ka, Ke, Km, Ky, Lv, My, Po, Ri, Sk, Su, Te, Vn, Vl, Zh.

Dicranella ruféscens (Dicks.) Schimp.- [CAM, DF, FSZ, CRM], If, Kr, Lv, Sk, Su. Vn, Zh.

Dicranella schreberiána (Hedw.) Dix. - [CAM, MF, DF, FSZ], Ck, Cv, If, Ky, Lv, Sk, Te, Vn, Zh.

Dicranella subuláta (Hedw.) Schimp. (D. curvata (Hedw.) Schimp.) - [CAM, DF], Cv, If, Lv, Sk, Te.

Dicranella vária (Hedw.) Schimp. - [CAM, MF, DF, FSZ, SZ, CRM], Ch, Ck, Cv, If, $\mathrm{Ka}, \mathrm{Km}, \mathrm{Kr}, \mathrm{Ky}, \mathrm{Lv}, \mathrm{Od}, \mathrm{Po}, \mathrm{Ri}, \mathrm{Sk}, \mathrm{Sp}, \mathrm{Su}, \mathrm{Te}, \mathrm{Vn}, \mathrm{Vl}$.

Dicranodóntium aspérulum (Mitt.) Broth. - [CAM], If.

Dicranodontium denudátum (Brid.) Britton (D. longirostre Bruch \& Schimp.) - [CAM, MF, DF, FSZ, CRM], Cv, If, Ky, Lv, Ri, Sk, Su,Vl, Vn, Zh.

Dicranowéisia cirráta (Hedw.) Lindb. - [CAM, DF, FSZ, SZ, CRM], If, Ke, Kr, Su.

Dicránum bonjeánii De Not. - [CAM, MF, DF, FSZ, SZ], Ch, Ck, Cv, If, Ka, Km, Ky, Lu, Lv, My, Po, Ri, Sk, Su, Te, Vn, Vl, Zh.

Dicranum elongátum Schleich. ex Schwaegr. (D. sendtneri Limpr.) - [CAM], If, Sk.

Dicranum flagelláre Hedw. (Orthodicranum flagellare (Hedw.) Loeske) - [CAM, MF, DF, FSZ], Ch, Ck, Cv, If, Ka, Ky, Lv, Po, Ri, Sk, Su, Vn, Vl, Zh.

Dicranum flexicáule Brid. (D. fuscescens var. flexicaule (Brid.) Wilson) - [CAM, FSZ], If, Sk, Vn. 
Dicranum fúlvum Hook. (Orthodicranum fulvum (Hook.) G.Roth ex Casares-Gil) [CAM, MF, DF], If, Ky, Lv, Sk, Vn.

Dicranum fuscéscens Sm. - [CAM, MF, SZ], Cv, Dn, If, Sk, Zh.

Dicranum groenlándicum Brid. (D. elongatum subsp. groenlandicum (Brid.) Mönk.) [CAM], Sk.

Dicranum május Sm. - [CAM, CRM], Kr, Lv, Sk.

Dicranum montánum Hedw. (Orthodicranum montanum (Hedw.) Loeske - [CAM, MF, DF, FSZ, SZ], Ch, Ck, Cv, Dn, Do, If, Ka, Km, Ky, Kr, Lu, Lv, Od, Po, Ri, Sk, Su, Vn, $\mathrm{Vl}, \mathrm{Zh}$.

Dicranum muehlenbéckii Bruch \& Schimp. - [CAM, CRM], If, Kr, Sk.

Dicranum polysétum Sw. (D. rugosum Brid.) - [CAM, MF, DF, FSZ, SZ], Ch, Ck, Cv, Dn, Do, If, Ka, Km, Ky, Lu, Lv, Po, Ri, Sk, Su, Vl,Vn, Zh.

Dicranum scopárium Hedw. - [CAM, MF, DF, FSZ, SZ, CRM], Ch, Ck, Cv, Dn, Do, If, Ka, Km, Ki, Kr, Ky, Lu, Lv, My, Od, Po, Ri, Sk, Sp, Su, Te, Vl, Vn, Zh.

Dicranum spadíceum Zett. (D. muehlenbeckii var. spadiceum (J.E.Zetterst.) Podp.) [CAM], If, Sk.

Dicranum spúrium Hedw. - [CAM, MF, DF], If, Lv, Ri. Sk, Zh.

Dicranum táuricum Sap. (Orthodicranum tauricum (Sapjegin) Smirnova) - [CAM, MF, FSZ, SZ, CRM], Ch, Ck, Do, If, Kr, Ky, Lu, Lv, Vn.

Dicranum undulátum Schrad. ex Brid. (D. bergeri Blandow, D. affine Funck) - [CAM], If, Sk.

Dicranum víride (Sull. \& Lesq.) Lindb. (D. fulvum var. viride Grout) - [CAM, MF, DF, FSZ, CRM], Ch, Ck, Cv. If, Ky, Lv, Po, Ri, Sk, Su, Te, Vl, Vn, Zh.

Didýmodon acútus (Brid.) Saito (Barbula acuta (Brid.) Brid.) - [CAM, MF, DF, FSZ, SZ, CRM], Cv, Dn, Do, If, Ka, Ke, Km, Kr, Lu, My, Po, Ri, Sk.

Didimodon cordátus Jur. (Barbula cordata (Jur.) Loeske) - [CRM], Kr.

Didymodon fállax (Hedw.) Zander (Barbula fallax Hedw.) - [CAM, MF, DF, FSZ, SZ, $\mathrm{CRM}$ ], Ch, Ck, Cv, Do, If, Ka, Ki, Ke, Km, Kr, Ky, Lv, Po, Ri, Sk, Su, Te, Vn, Zh.

Didymodon ferrugíneus (Schimp. ex Besch.) M. Hill (Barbula reflexa (Brid.) Brid.) [CAM, MF, DF, FSZ], Cv, If, Lv, Ri, Te.

Didymodon insulánus (De Not.) M. Hill (Barbula cylindrica (Taylor) Schimp.) - [CAM, DF, SZ, CRM], If, Ke, Kr, Sk, Te.

Didymodon lúridus Hornsch.(Barbula lurida Hornsch.) - [CAM, DF, CRM], If, Kr, Sk, Te.

Didymodon rigídulus Hedw. (Barbula rigidula (Hedw.) Milde) - [CAM, DF, FSZ, SZ, CRM], Ck, Cv, Dn, Do, If, Ka, Ki, Km, Kr, Lu, Lv, My, Od, Sk, Te, Vn.

Didymodon sinuósus (Mitt.) Delogne (Barbula sinuosa (Mitt.) Grav.) - [SZ, CRM], Ke, $\mathrm{Kr}$.

Didymodon spadíceus (Mitt.) Limpr. (Barbula spadicea (Mitt.) Braithw.) - [CAM, DF, $\mathrm{SZ}, \mathrm{CRM}], \mathrm{Cv}$, If, Ki, Kr, Lv, Sk, Te.

Didymodon topháceus (Brid.) Lisa (Barbula tophacea (Brid.) Mitt.) - [CAM, DF, FSZ, $\mathrm{SZ}, \mathrm{CRM}$, Ck, Cv, Do, If, Ka, Ki, Km, Kr, Lv, Od, Sk, Te.

Didymodon vineális (Brid.) Zander (Barbula vinealis Brid.) - [CAM, MF, DF, FSZ, SZ, $\mathrm{CRM}], \mathrm{Ch}, \mathrm{Ck}, \mathrm{Cv}$, Do, If, Ka, Ke, Kr, Ky, Lu, Lv, My, Po, Ri, Sk, Su,Te.

Diphyscium foliósum (Hedw.) Mohr - [CAM, DF, CRM], If, Kr, Lv, Sk.

Distíchium capilláceum (Hedw.) Bruch \& Schimp. - [CAM, MF, DF, CRM], Cv, If, Km $\mathrm{Kr}, \mathrm{Ky}, \mathrm{Lv}, \mathrm{Sk}$, Te.

Distichium inclinátum (Hedw.) Bruch \& Schimp. (Cynodontium inclinatum (Hedw.) Bruch \& Schimp.) - [CAM], If.

Dítrichum flexicáule (Schwaegr.) Hampe (Cynodontium flexicaule (Schwaegr.) Hampe, Didymodon flexicaule Schleirch. - [CAM, DF, FSZ, CRM], Cv, If, Km, Kr, Lv, Sk, Vn. 
Ditrichum heteromállum (Hedw.) Britton - [CAM, DF, SZ], Cv, Dn, If, Lv, Sk, Te. Ditrichum pállidum (Hedw.) Hampe - [CAM, DF], Cv, Lv, Sk, Te.

Ditrichum pusíllum (Hedw.) Hampe (D. tortile (Schrad.) Brockm.) - [CAM, MF, FSZ, SZ], Cv, Dn, If, Ka, Lv, Pi, Sk, Zh.

Ditrichum subulátum (Bruch) Hampe (Trichostomum subulatum Bruch) - [CAM], Cv.

Ditrichum zonátum (Brid.) Kindb. (D. heteromallum var. zonatum (Brid.) Podp.) [CAM], Sk.

Drepanocládus adúncus (Hedw.) Warnst. - [CAM, MF, DF, FSZ, SZ, CRM], Ch, Ck, $\mathrm{Cv}$, Dn, Do, If, Ka, Ke, Ki, Km, Kr, Ky, Lu, Lv, Po, Ri, Sk, Su, Te, Vl, Vn, Zh.

Drepanocladus lycopodióides (Brid.) Warnst. (Pseudocalliergon lycopodioides (Brid.) Hedenäs) - [CAM, MF, DF], If, Ky, Lv, Ri, Vl, Zh.

Drepanocladus polycárpos (Blandow ex Voit) Warnst. (D. aduncus var. polycarpus (Bland.) Moenk.) - [MF], Ch, Ka, Ky.

Drepanocladus polýgamus (Schimp.) Hedenäs (Campylium polygamum (Schimp.) Lange \& C.E.O.Jensen) - [CAM, MF, DF, FSZ, SZ], Ch, Ck, Cv, Do, If, Ka, Ki, Ky, Po, Ri, Te, $\mathrm{Vn}, \mathrm{Vl}$.

Drepanocladus séndtneri (Schimp. ex H. Müll.) Warnst. - [CAM, MF, DF, FSZ, SZ, CRM], Ch, Dn, If, Ka, Ke, Kr, Ky, Lv, My, Od, Po, Sk, Su, Te, Vl, Zh.

Drepanocladus trifárius (F. Weber \& D. Mohr) Brotherus ex Paris (Pseudocalliergon trifarium (F.Weber \& Mohr) Loeske, Calliergon trifarium (F.Weber \& D.Mohr) Kindb.) [MF, DF], Ch, Ky, Lv, Vl.

Encalýpta ciliáta Hedw. - [CAM, MF, DF, FSZ], Cv, If, Ky, Lv, Ri, Sk, Vn, Zh.

Encalypta longícolla Bruch - [CAM], Sk.

Encalypta mútica Hag. (E. vulgaris var. mutica Brid.) - [SZ], Do, My.

Encalypta rhaptocárpa Schwaegr. - [CRM], If, Kr, Sk.

Encalypta spathuláta H. Müll. (E. rhaptocarpa var. spathulata (Müll.Hal.) Husn.) - [SZ, CRM], Do, Kr, Lu.

Encalypta streptocárpa Hedw. (E. contorta Hopp. ex Lindb.) - [CAM, DF, FSZ, SZ, CRM], Cv, Do, If, Km, Kr, Lu, Lv, Ri, Sk, Te, Vl,Vn.

Encalypta vulgáris Hedw. - [CAM, DF, FSZ, SZ, CRM], Ck, Cv, Dn, Do, If, Ka, Ke, $\mathrm{Km}, \mathrm{Kr}, \mathrm{Ky}, \mathrm{Lu}, \mathrm{Lv}, \mathrm{My}$, Od, Po, Ri, Sk, Sp, Su, Te, Vn, Zh.

Éntodon concínnus (De Not.) Par. - [CAM, FSZ, CRM], Cv, If, Kr, Sk, Vn.

Entósthodon fasciculáris (Hedw.) H. Müll. (Funaria fascicularis (Hedw.) Lindb.) [CAM, MF, DF, FSZ, SZ], Ky, Lv, My, Pi, Sk, Vn.

Entosthodon hungáricus (Boros) Loeske (Funaria hungarica Boros) - [SZ, CRM], Ck, $\mathrm{Cv}, \mathrm{Dn}$, Do, Ke, Km, Kr, My, Sp.

Entosthodon muhlenbérgii (Turn.) Fife (Funaria dentata Crome, Funaria muhlenbergii Turner) - [FSZ, SZ, CRM], Dn, Kr, Ky, Te.

Ephemerum crassinérvium (Schwagr.) Hampe subsp. séssile (Bruch) Holyoak (Ephemerum sessile (Bruch) H. Müll.) - [FSZ], Vn.

Ephemérum minutíssimum Lindb. - [FSZ], Vn.

Ephemerum recurvifólium (Dicks.) Boulay - [FSZ], Vn.

Ephemerum serrátum (Hedw.) Hampe - [CAM, MF, DF, FSZ], Ch, Lv, Sk, Te, Vn.

Eucládium verticillátum (With.) Bruch \& Schimp. - [CAM, DF, FSZ, SZ, CRM], Cv, Do, If, Km, Kr, Od.

Eurhynchiástrum pulchéllum (Hedw.) Ignatov \& Huttunen (Eurhynchium pulchellum (Hedw.) Jenn. ) - [CAM, MF, DF, FSZ, SZ, CRM], Ch, Ck, Cv, Dn, Do, If, Kr, Ky, Lv, My, Po, Ri, Sk, Sp, Su, Vl, Vn, Zh.

Eurhynchiástrum pulchéllum (Hedw.) Ignatov \& Huttunen var. práecox (Hedw.) Ochyra \& Żarnowiec - [CAM, MF], Ch, Cv. 
Eurhýnchium angustiréte (Broth.) T. Kop. (E. zetterstedtii Stormer) - Ch, Cv, If, Kr, Ky, Lv, Po, Sk, Su, Te, Vl, Zh. - [CAM, MF, DF, FSZ, CRM].

Eurhynchium striátum (Hedw.) Schimp.) - [CAM, MF, DF, FSZ, CRM], Ch, Ck, Cv, If, Km, Kr, Ky, Lv, Po, Ri, Sk, Te, Vl, Vn, Zh.

Exsertothéca críspa (Hedw.) S.Olsson, Enroth \& D.Quandt (Neckera crispa Hedw.) [CAM, DF, CRM], Cv, If, Km, Kr, Lv, Sk.

Físsidens adianthóides Hedw. - [CAM, MF, DF, FSZ, SZ], Ch, Ck, Cr, If, Km, Kr, Lv, Sk, Su, Te, Vn, Vl, Zh.

Fissidens arnóldii Ruthe (F. obtusifolius Wils.) - [CAM, MF, DF], Cv, Te, Zt.

Fissidens bryóides Hedw. (Fissidens exiguus Sull., Fissidens incurvus Starke ex Röhl.) [CAM, MF, DF, FSZ, SZ, CRM], Ch, Ck, Cv, Dn, If, Ka, Km, Kr, Ky, Lv, Lu, Po, Ri, Sk, $\mathrm{Su}, \mathrm{Te}, \mathrm{Vn}, \mathrm{Zh}$.

Fissidens crássipes Wils. ex Bruch \& Schimp. (F. mildeanus Schimp.) - [MF, FSZ, SZ, $\mathrm{CRM}], \mathrm{Ck}, \mathrm{Kr}, \mathrm{Mk}, \mathrm{Od}, \mathrm{Vn}, \mathrm{Zh}$.

Fissidens críspus Mont. (Fissidens minutulus Sull.) - [CAM, DF, FSZ, SZ, CRM], Cv, $\mathrm{Km}, \mathrm{Kr}, \mathrm{Lu}, \mathrm{Te}, \mathrm{Vn}$.

Fissidens dúbius P. Beauv. (F. cristatus Wilson ex Mitt., F. decipiens De Not.) - [CAM, DF, FSZ, SZ, CRM], Cv, If, Kr, Lv, Ri, Sk, Te, Vn.

Fissidens éxilis Hedw.- [CAM, DF, FSZ], Cv, Lv, Sk, Te, Vn.

Fissidens fontánus (Bach. Pyl.) Steud. (Octodiceras fontanum (Bach.Pyl.) Lindb.) [MF], Zh.

Fissidens gymnándrus Büse ( $F$. bryoides var. gymnandrus (Büse) Ruthe) - [CAM, MF], $\mathrm{Cv}$, If, Sk, Vl.

Fissidens marginátulus Meln. - [CAM, MF, FSZ, CRM], Ck, Cv, If, Ki, Km, Kr, Ky, Lv, Ri, Sk, Te, Vn.

Fissidens osmundóides Hedw. - [CAM, DF], Lv.

Fissidens pusíllus (Wilson) Milde (Fissidens viridulus var. pusillus Wilson) - [CAM, MF, DF], Cv, If, Km, Lw, Sk, Te, Zt.

Fissidens rivuláris (Spruce) Schimp. $-[\mathrm{CRM}], \mathrm{Kr}$.

Fissidens rúfulus Bruch \& Schimp. - [CAM], Cv.

Fissidens taxifólius Hedw. [CAM, MF, DF, FSZ, SZ, CRM], Ck, Cv, Dn, If, Ka, Km, $\mathrm{Kr}, \mathrm{Ky}, \mathrm{Lv}, \mathrm{Od}, \mathrm{Po}, \mathrm{Ri}, \mathrm{Su}, \mathrm{Te}, \mathrm{Vn}, \mathrm{Vl}, \mathrm{Zh}$.

Fissidens virídulus (Sw.) Wahlenb. - [CAM, MF, DF, FSZ, SZ, CRM], Ck, Cv, If, Ka, Lu, Lv, Po, Su, Te, Vn.

Fontinális antipyrética Hedw. - [CAM, MF, DF, FSZ, SZ, CRM], Ch, Ck, Cv, Dn, If, $\mathrm{Ka}, \mathrm{Ki}, \mathrm{Kr}, \mathrm{Ky}, \mathrm{Lu}, \mathrm{Lv}, \mathrm{My}, \mathrm{Po}, \mathrm{Ri}, \mathrm{Sk}, \mathrm{Su}, \mathrm{Vl}, \mathrm{Vn}, \mathrm{Zh}$.

Fontinalis hypnóides C. Hartm. - [CAM, MF, SZ], Dn, If, Ke, Sk, Su, Zh.

Funária hygrométrica Hedw. - [CAM, MF, DF, FSZ, SZ, CRM], Ch, Ck, Cv, Dn, Do, If, Ka, Ke, Ki, Km, Kr, Ky, Lu, Lv, My, Od, Po, Ri, Sk, Sp, Su, Te, Vn, Vl, Zh.

Funaria micróstoma Bruch ex Schimp. - [DF], Lv.

Grímmia alpéstris (F.Weber \& Mohr) Schleich. - [CAM], If, Sk.

Grimmia anódon Bruch \& Schimp. (Schistidium anodon (Bruch\& Schip.) Loeske) [MF, DF, FSZ, SZ, CRM], Ch, Dn, Km, Kr, Lu, Lv, My, Vn, Zh.

Grimmia anómala Hampe ex Schimp. - [CAM], If, Sk.

Grimmia criníta Brid. - [SZ], Kr.

Grimmia decípiens (Schultz) Lindb. - [CAM, CRM], Kr, Sk.

Grimmia donniána Sm. - [CAM], If, Sk,

Grimmia elátior Bruch ex Bals. - Criv. \& De Not. (Dryptodon incurvus (Hornsch.) Brid.) - [CAM], If, Sk.

Grimmia elongáta Kaulf. - [CAM, CRM], Sk, If, Kr.

Grimmia funális (Schwaegr.) Bruch \& Schimp. - [CAM], Sk, If. 
Grimmia fuscolútea Hook. - [CAM], Cv.

Grimmia hártmanii Schimp.- [CAM, FSZ, CRM], If, Kr, Lv, Sk, Vn.

Grimmia incúrva Schwaegr. - [CAM], If, Sk.

Grimmia laevigáta (Brid.) Brid. (G. campestris Burchel ex Hook.) - [CAM, MF, DF, FSZ, SZ, CRM], Ck, Dn, Do, If, Ka, Ki, Km, Kr, Ky, Lu, My, Sk, Sp, Te, Vn, Zh.

Grimmia longiróstris Hook. (Grimmia ovalis (Hedw.) Lindb., G.commutata Hüben., G. ovata Schwägr.) - [CAM, MF, FSZ, SZ, CRM], Ck, Dn, Do, If, Ki, Kr, Ky, Lu, My, Ri, $\mathrm{Sk}, \mathrm{Sp}, \mathrm{Vn}, \mathrm{Zh}$.

Grimmia montána Bruch \& Schimp. - [CAM], If.

Grimmia muehlenbéckii Schimp.- [CAM, MF, FSZ, SZ, CRM], Kr, Sk, Sp, Zh.

Grimmia orbiculáris Bruch ex Wils. - [DF, CRM], Kr, Te.

Grimmia plagiopódia Hedw.- [DF, FSZ, SZ], Dn, Km, Lu, Sp, Vn.

Grimmia poecilóstoma Cardot \& Sebille - [CRM], Kr.

Grimmia pulvináta (Hedw.) Sm.- [CAM, MF, DF, FSZ, SZ, CRM], Ch, Ck, Cv, Dn, Do, If, Ka, Ke, Ki, Km, Kr, Ky, Lu, Lv, My, Od, Po, Ri, Sk, Sp, Su, Te, Vn, Vl, Zh.

Grimmia ramóndii (Lam. \& DC.) Marg. (Grimmia patens (Hedw.) B.S.G. - [CAM], Sk.

Grimmia tergéstina Tomm. ex Bruch \& Schimp. (G. tergestinoides Culm.) - [DF, CRM], Km, Kr.

Grimmia trichophýlla Grev. (G. meridionalis (Müll. Hal.)E.Maier) - [CAM, MF, SZ, CRM], Do, Kr, Ri. Sk, Zh.

Gymnostomum aeruginósum Sm. (Weisia rupestris C. Müll.) - [CAM, DF, CRM], Cv, If, $\mathrm{Kr}, \mathrm{Sk}, \mathrm{Te}$.

Gymnóstomum calcáreum Nees \& Hornsch. - [DF, CRM], Kr, Te.

Gyrowéisia ténuis (Hedw.) Schimp. - [CAM, DF, CRM], Cv, If, Kr, Lv, Sk, Te.

Hamatocáulis vernicósus (Mitt.) Hedenäs (Drepanocladus vernicosus (Mitt.) Warnst.) [CAM, MF, DF, FSZ], Ch, Ck, If, Ky, Lv, Ri, Sk, Su, Te, Vn, Vl, Zh.

Haplocládium microphýllum (Hedw.) Broth. - [FSZ], Od.

Hedwígia ciliáta (Hedw) P.Beauv.- [CAM, MF, DF, FSZ, SZ, CRM], Ck, Cv, Dn, Do, If, Ki, Km, Kr, Ky, Lu, Lv, My, Od, Ri, Sk, Sp, Vn, Zh.

Helódium blandówii (F.Weber \& Mohr) Warnst. - [MF, DF, FSZ], Ch, Ki, Ky, Lv, Ri, Vl.

Hennediélla héimii (Hedw.) Zander (Desmatodon heimii (Hedw.) Mitt.) - [DF, FSZ], Ck, Lv.

Herzogiélla seligéri (Brid.) Iwats. (Dolichotheca seligerii (Brid.) Loeske) - [CAM, MF, DF, FSZ, SZ, CRM], Ch, Ck, Cv. If, Ka, Ki, Km, Kr, Ky, Lu, Lv, Sk, Te, Vl, Vn, Zh.

Herzogiella striatélla (Brid.) Iwats.(Dolichotheca striatela (Brid.) Iwats.) - [CAM], If, Sk.

Heterocládium dimórphum (Brid.) Schimp.- [CAM], If, Sk.

Heterocladium heterópterum (Brid.) Schimp. - [CAM], If, Sk.

Heterophýllium affíne (Hook.) Fleisch. (H. nemorosum (Brid.) Kindb.) - [CAM, DF], If, Lv, Sk.

Homália trichomanóides (Hedw.) Brid. - [CAM, MF, DF, FSZ, SZ, CRM], Ch, Ck, Cv, Dn, If, Ka, Ki, Km, Kr, Ky, Lu, Lv, My, Od, Po, Ri, Sk, Su, Te, Vl, Vn, Zh.

Homalothécium aúreum (Spruce) Robins. (Camptothecium aureum (Spruce) Bruch \& Schimp.) - [CRM], Kr.

Homalothecium lutéscens (Hedw.) Robins. (Camptothecium lutescens (Hedw.) Schimp.) - [CAM, MF, DF, FSZ, SZ, CRM], Ch, Ck, Cv, Dn, Do, Ka, Ke, Ki, Km, Kr, Ky, Lu, Lv, $\mathrm{My}, \mathrm{Od}, \mathrm{Po}, \mathrm{Sk}, \mathrm{Sp}, \mathrm{Su}, \mathrm{Te}, \mathrm{Zh}$.

Homalothecium philippeánum (Spruce) Schimp. - Cv, If, Km, Kr, Lv, Ri, Sk, Te. 
Homalothecium seríceum (Hedw.) Schimp. - [CAM, DF, FSZ, SZ, CRM], Ch, Ck, Cv, Dn, Do, If, Ka, Ke, Ki, Km, Kr, Ky, Lu, Lv, My, Od, Po, Ri, Sk, Sp, Te, Vn, Zh.

Homomállium incurvátum (Schrad. ex Brid.) Loeske - [CAM, MF, DF, FSZ, SZ, CRM], Ch, Cv, Do, If, Km, Kr, Lu, Lv, Ri, Sk, Te.

Hookéria lúcens (Hedw.) - [CAM], If, Lv, Sk.

Hygroamblystégium fluviátile (Hedw.) Loeske - [CAM, MF, DF], Km, Sk, Zh.

Hygroamblystegium húmile (P. Beauv.) Vanderp., Goffinet \& Hedenäs (Amblystegium humile (P.Beauv.) Crundw., A. kochii Bruch \& Schimp., Leptodictyum humile (P.Beauv.) Ochyra, Leptodictyum kochii (Schimp.) Warnst.) - [CAM, MF, DF, FSZ, SZ], Ch, Ck, Dn, Do, Ka, Ke, Ky, Lu, Lv, Po, Sk, Su, Te, Zh.

Hygroamblystégium ténax (Hedw.) Jenn. (Amblystegium tenax (Hedw.) C.E.O.Jensen) [CAM, MF, DF, SZ, CRM], Ck, Cv, Do, Ki, Ky, Lv, Mk, Sk, Te.

Hygroamblystegium várium (Hedw.) Mönk. (Amblystegium varium (Hedw.) Lindb.) [CAM, MF, DF, FSZ, SZ, CRM], Ch, Ck, Cv, Dn, Do, If, Ka, Ke, Ki, Km, Kr, Ky, Lu, Lv, My, Od, Po, Ri, Sk, Sp, Su, Te, Vn, Vl, Zh.

Hygrohýpnum duriúsculum (De Not.) D.W. Jamieson - [CAM], If, Sk.

Hygrohypnum lúridum (Hedw.) Jenn. - [CAM, DF, FSZ, CRM], Cv, If, Km, Kr, Ky, Sk, Te, Vn.

Hygrohypnum mólle (Hedw.) Loeske - [CAM], If, Sk.

Hygrohypnum ochráceum (Turn. ex Wils.) Loeske - [CAM], If, Sk.

Hylocomiástrum pyrenáicum (Spruce) Fleisch. - [CAM], If, Sk.

Hylocomiastrum umbrátum (Hedw.) Fleisch. - [CAM], If, Sk.

Hylocómium spléndens (Hedw.) Schimp. - [CAM, MF, DF, FSZ, CRM], Ch, Cv, If, Ka, $\mathrm{Km}, \mathrm{Kr}, \mathrm{Ky}, \mathrm{Lv}, \mathrm{Po}, \mathrm{Sk}, \mathrm{Su}, \mathrm{Te}, \mathrm{Vl}, \mathrm{Vn}, \mathrm{Zh}$.

Hymenolóma compactum (Schwagr.) Ochyra (Dicranoweisia compacta (Schleich. ex Schwaegr.) Schimp., D. crispula var. compacta (Schwägr.) Lindb.) - [CAM], Sk.

Hymenoloma críspulum (Hedw.) Ochyra (Dicranoweisia crispula (Hedw.) Milde) [CAM, MF], If, Km, Sk.

Hymenostýlium recurviróstrum (Hedw.) Dix. (Gymnostomum recurvirostrum Hedw.) [CAM], Sk.

Hýpnum ándoi Sm. (H. cupressiforme var. mammillatum Brid.) - [CAM, MF], Cv, Ri.

Hypnum bámbergeri Schimp. - [CAM], Cv, Sk.

Hypnum callíchroum Brid. - [CAM, DF], If, Lv, Sk.

Hypnum cupressifórme Hedw. - [CAM, MF, DF, FSZ, SZ, CRM], Ch, Ck, Cv, Dn, Do, If, $\mathrm{Ka}, \mathrm{Ke}, \mathrm{Ki}, \mathrm{Km}, \mathrm{Kr}, \mathrm{Ky}, \mathrm{Lu}, \mathrm{Lv}, \mathrm{My}$, Od, Po, Ri, Sk, Sp, Su, Te, Vn, Vl, Zh.

Hypnum cupressifórme Hedw. var. filifórme Brid. - [CAM, SZ], Cv, If, Ka, My, Sk.

Hypnum cupressifórme Hedw. var. lacunósum Brid. - [CAM, MF, DF, SZ, CRM], Cv, $\mathrm{Kr}, \mathrm{Ri}, \mathrm{Te}, \mathrm{Vl}$.

Hypnum cupressifórme Hedw. var. subjuláceum Molendo - [CAM, SZ, CRM], Cv, Do, If, $\mathrm{Kr}, \mathrm{Lu}, \mathrm{My}$, Sk.

Hypnum fértile Sendt. - [CAM, MF, DF], Cv, If, Lv, Ri, Sk, Te, Zh.

Hypnum hamulósum Schimp. - [CAM], Cv, If, Sk.

Hypnum impónens Hedw. - [CAM, MF, DF, FSZ,CRM], Cv, If, Km, Kr, Lv, Sk, Zh.

Hypnum jutlándicum Holmen \& Warncke (H. cupressiforme var. ericetorum Schimp.) [MF], V1.

Hypnum palléscens (Hedw.) P. Beauv. (Hypnum reptile Rich.) - [CAM, MF, DF, FSZ, $\mathrm{SZ}, \mathrm{CRM}$ ], Ch, Ck, Cv, Dn, If, Ka, Ki, Km, Kr, Ky, Lu, Lv, Od, Po, Ri, Sk, Su, Te, Vl, $\mathrm{Vn}, \mathrm{Zh}$.

Hypnum recurvátum (Lindb. \& Arnell) Kindb. (H. fastigiatum Brid.) - [CAM, DF], $\mathrm{Cv}$, If, Lv, Sk, Te.

Hypnum revolútum (Mitt.) Lindb. - [CAM, CRM], Cv, If, Kr, Sk. 
Hypnum vauchéri Lesq. (H. cupressiforme var. vaucheri C.Jens.) - [CAM, DF, FSZ, SZ, CRM], Cv, If, Ka, Ke, Kr, Lu, Lv, My, Od, Te.

Imbribrýum alpinum (Huds. ex With.) N. Pedersen (Bryum alpinum Huds. ex With.) [CAM, MF, FSZ, SZ, CRM], Ch, Ck, Do, Kr, Ky, Mk, Sk, Sp.

Imbribryum mildeánum (Jur.) J.R. Spence (Bryum mildeanum Jur., B. alpinum var. mildeanum (Jur.) Podp.) - [CAM], If, Sk.

Isopterygiópsis muelleriána (Schimp.) Iwats. (Isopterygium muellerianum (Schimp.) Lindb.) - [CAM], Cv, If, Sk.

Isopterygiopsis pulchélla (Hedw.) Iwats. (Isopterygium pulchellum (Hedw.) A.Jaeger.) [CAM, DF], Cv, If, Lv, Po, Sk, Te.

Isothécium alopecuróides (Lam. ex Dubois) Isov. (I. myurum Brid., I. viviparum Lindb.) - [CAM, MF, DF, FSZ, SZ, CRM], Cv, Dn, If, Ka, Ki, Km, Kr, Ky, Lv, Po, Ri, Sk, Te, $\mathrm{Vl}, \mathrm{Vn}, \mathrm{Zh}$.

Isothecium hóltii Kindb. (Isothecium myosuroides var. rivulare Limpr.) - [CAM], Cv.

Isothecium myosuróides Brid. - [CAM, MF], Cv, If, Sk.

Kiaéria blýttii (Bruch \& Schimp.) Broth. - [CAM], If, Sk.

Kiaeria falcáta (Hedw.) Hag. - [CAM], If, Sk.

Kiaeria stárkei (F.Weber \& Mohr) Hag. - [CAM], If, Sk.

Kindbérgia praelónga (Hedw.) Ochyra (Eurhynchium praelongum (Hedw.) Schimp.) [CAM, MF, DF, FSZ, SZ], Ck, Cv, Do, If, Ke, Kr, Lv, Po, Sk, Sp, Su, Te, Vn.

Lazarénkia kozlóvii (Lazar.) Boiko (Pterygoneurum kozlovii Lazar.) - [SZ], Kr, Od, Sp.

Leptóbryum pyrifórme (Hedw.) Wils. - [CAM, MF, DF, FSZ, SZ], Ch, Ck, Cv, Dn, Do, If, Ka, Ke, Ki, Km, Ky, Lu, Lv, My, Od, Po, Ri, Sk, Sp, Su, Te, Vn, Zh.

Leptodíctyum ripárium (Hedw.) Warnst. (Amblystegium riparium (Hedw.) Schimp.) [CAM, MF, DF, FSZ, SZ, CRM], Ch, Ck, Cv, Dn, Do, If, Ka, Ke, Ki, Km, Kr, Ky, Lu, Lv, My, Od, Po, Ri, Sk, Su, Te, Vn, Vl, Zh.

Leptódon smíthii (Hedw.) F.Weber \& Mohr - [SZ, CRM], Kr.

Lescúraea incurvata (Hedw.) E. Lawto (L. atrovirens (Bruch \& Schimp.) E.Lawto, Pseudoleskea incurvata (Hedw.) Loeske) - [CAM, SZ, CRM], Dn, If, Ke, Ki, Kr, Lv, Sk.

Lescuraea mutábilis (Brid.) Lindb. ex Hag. (Leskea mutabilis (Brid.) Boul.) - [CAM], If, Sk.

Lescuraea pátens Lindb. (Pseudoleskea patens (Lindb.) Kindb.) - [CAM], Sk.

Lescuraea plicáta (Schleich. ex F.Weber \& D.Mohr) Broth (Ptychodium plicatum (Schleich. ex F.Weber \& Mohr) Schimp.) - [CAM], If, Sk.

Lescuraea radicósa (Mitt.) Mönk. (Pseudoleskea radicosa (Mitt.) Macoun \& Kindb.) [CAM], Cv, If, Sk.

Lescuraea saviána (De Not.) E. Lawton (Pseudoleskea saviana (De Not.) Latzel, Ptychodium tauricum Sap.) - [CAM, CRM], Kr, Sk.

Lescuraea saxícola (Schimp.) Mol. (Lescuraea mutabilis var. saxicola (Bruch \& Schimp.)Hag.) - [CAM], If, Sk.

Leskea polycárpa Hedw. ) - [CAM, MF, DF, FSZ, SZ, CRM], Ch, Ck, Cv, Dn, Do, If, $\mathrm{Ka}, \mathrm{Ke}, \mathrm{Ki}, \mathrm{Km}, \mathrm{Kr}, \mathrm{Ky}, \mathrm{Lu}, \mathrm{Lv}, \mathrm{My}, \mathrm{Od}, \mathrm{Po}, \mathrm{Ri}, \mathrm{Sk}, \mathrm{Sp}, \mathrm{Su}, \mathrm{Te}, \mathrm{Vn}, \mathrm{Vl}, \mathrm{Zh}$.

Leucóbryum gláucum (Hedw.) Ångstr. - [CAM, MF, DF, FSZ, SZ, CRM], Ch, Ck, Cv, If, Ka, Km, Ky, Lv, Po, Ri, Sk, Su, Te, Vl, Vn, Zh.

Leucobryum juniperoídeum (Brid.) H. Müll. (L. minus Hampe) - [CAM], Cv, If, Lv, Sk.

Leucodon sciuróides (Hedw.) Schwaegr. - [CAM, MF, DF, FSZ, SZ, CRM], Ch, Ck, Cv, Dn, Do, If, Ka, Ke, Ki, Km, Kr, Ky, Lu, Lv, My, Od, Po, Ri, Sk, Sp, Su, Te, Vn, Vl, Zh.

Loeskeóbryum breviróstre (Brid.) Fleisch. (Hylocomium brevirostre (Brid.) Schimp.) [CAM, CRM], If, Kr, Sk.

Meésia longiséta Hedw. - [MF, FSZ], Ky, Vn. 
Meesia triquétra (L. ex Jolycl.) Ångstr. - [CAM, MF, DF, FSZ], If, Ky, Lv, Pi, Vl, Vn, $\mathrm{Zh}$.

Meesia uliginósa Hedw.- [CAM, FSZ], If, Sk, Vn.

Micróbryum curvícollum (Hedw.) Zander (Phascum curvicollum Hedw.) - [DF, SZ],

Ke, Lv.

Microbryum davalliánum (Sm.) Zander (Pottia davalliana (Sm.) C.E.O.Jensen) [CAM, MF, DF, FSZ, SZ], Ch, Cv, Dn, Do, Ky, Lv, Su, Te, Vn.

Microbryum starckeánum (Hedw.) Zander (Pottia starckeana (Hedw.) Müll.Hal.).

Mníum hórnum Hedw. - [CAM, MF, DF, CRM], Km.Kr, Lv, Sk.

Mnium lycopodióides Schwaegr. - [CAM, MF, DF], Cv, If, Km, Ky, Lv, Sk, Te, Vn, Zh.

Mnium marginátum (Dicks.) P. Beauv. - [CAM, MF, DF, FSZ, CRM], Ch, Ck, Cv, If, $\mathrm{Ki}, \mathrm{Km}, \mathrm{Kr}, \mathrm{Ky}, \mathrm{Lv}, \mathrm{Po}, \mathrm{Sk}, \mathrm{Su}, \mathrm{Te}, \mathrm{Vn}, \mathrm{Zh}$.

Mnium spinósum (Voit) Schwaegr. - [CAM, CRM], Cv, If, Kr, Sk,

Mnium spinulósum Bruch \& Schimp. - [CAM], If, Sk.

Mnium stelláre Hedw. - [CAM, MF, DF, FSZ, CRM], Ch, Ck, Cv, If, Ki, Km, Kr, Ky, Lv, Po, Ri, Sk, Sp, Su, Te, Vl, Vn, Zh.

Mnium thómsonii Schimp. - [CAM, DF, CRM], Cv, If, Kr, Sk.

Molendóa hornschuchiána (Hook.) Lindb. ex Limpr. (Anoectangium hornschuchianum (Hook.) Funck ex Hornsch.) - [CAM], Cv.

Molendoa sendtneriána (Bruch \& Schimp.) Limpr. (Anoectangium sendtnerianum Bruch \& Schimp.) - [CAM], Sk.

Myrínia pulvinata (Wahlenb.) Schimp. - [FSZ], Ka.

Myurélla julácea (Schwaegr.) Schimp. - [CAM, CRM], If, Kr, Sk.

Myurella tenerríma (Brid.) Lindb. $-[\mathrm{CAM}]$, Sk .

Neckéra menziésii Drumm. (Metaneckera menziesii (Drumm.) Steere, Neckeradelphus menziesii (Hook.) Steere) - [CRM], Kr.

Neckera pennáta Hedw. - [CAM, MF, DF, FSZ, SZ, CRM], Ch, Cv, Dn, If, Ki, Km, Kr, $\mathrm{Lv}, \mathrm{Od}, \mathrm{Ri}, \mathrm{Sk}, \mathrm{Su}, \mathrm{Vl}, \mathrm{Vn}, \mathrm{Zh}$.

Neckera púmila Hedw. (N. fontinaloides Lindb.) - [CAM], If, Sk.

Nogoptérium grácile (Hedw.) Crosby \& W.R. Buck (Pterogonium gracile (Hedw.) Sm.) $-[\mathrm{CRM}], \mathrm{Kr}$.

Nyholmiélla gymnóstoma (Bruch ex Brid.) Holmen \& E. Warncke (Orthotrichum gymnostomum Bruch ex Brid.) - [CAM, MF, DF, FSZ, SZ, CRM], Ch, Do, If, Ka, Ky, $\mathrm{Kr}, \mathrm{Ri}, \mathrm{Sk}, \mathrm{Sp}, \mathrm{Vn}, \mathrm{Zh}$.

Nyholmiella obtusifólia (Schrad. ex Brid.) Holmen \& E. Warncke (Orthotrichum obtusifolium Brid.) - [CAM, MF, DF, FSZ, SZ, CRM], Ch, Ck, Cv, Do, If, Ka, Ke, Km, $\mathrm{Kr}, \mathrm{Ky}, \mathrm{Lv}, \mathrm{Od}, \mathrm{Po}, \mathrm{Ri}, \mathrm{Sk}, \mathrm{Su}, \mathrm{Te}, \mathrm{Vl}$.

Oligótrichum hercýnicum (Hedw.) Lam. \& DC. (Polytrichum hercynicum Hedw.) [CAM], Cv, If, Sk.

Oncóphorus vírens (Hedw.) Brid. (Cynodontium virens Schimp. ) - [CAM], If, Sk.

Oncophorus wahlenbérgii Brid. (Cynodontium wahlenbergii C.Hartm.) - [CAM], Sk.

Orthothécium intricátum (Hartm.) Schimp. (Holmgrenia intricata Lindb., Leskea intricata Hartm.) - [CAM, DF, CRM], Cv, If, Kr, Lv, Sk.

Orthothecium ruféscens (Dicks. ex Brid.) Schimp. - [CAM], If.

Orthótrichum affíne Schrad. ex Brid. (O. fastigiatum Bruch ex Brid.) - [CAM, MF, DF, FSZ, SZ, CRM], Ch, Ck, Cv, Dn, Do, If, Ka, Ke, Ki, Km, Kr, Ky, Lu, Lv, My, Od, Po, Ri, Sk, Sp, Su, Te, Vn, Vl, Zh.

Orthotrichum affine var. bohémicum Plašek \& Sawicki [CRM], Kr.

Orthotrichum alpéstre Bruch \& Schimp. - [CAM], Sk.

Orthotrichum anómalum Hedw. - [CAM, MF, DF, FSZ, SZ, CRM], Ck, Cv, Dn, Do, If, Ke, Ki, Km, Kr, Ky, Lu, Lv, My, Od, Ri, Sk, Sp, Te, Vn, Zh. 
Orthotrichum cupulátum Hoffm. ex Brid. (O. limprichtii I.Hagen, O. nudum Dicks) [CAM, MF, DF, FSZ, SZ, CRM], Ch, Cv, Do, If, Ke, Km, Kr, Lv, My, Te, Vn.

Orthotrichum cupulátum Hoffm. ex Brid. var. ripárium Huebener-[CRM], Kr.

Orthotrichum diaphánum Schrad. ex Brid. - Ck, If, Ka, Ke, Kr, Ky, Lv, My, Od, Ri, $\mathrm{Sk}, \mathrm{Sp}, \mathrm{Su}, \mathrm{Vn}$.

Orthotrichum lyéllii Hook. \& Taylor - [CAM, MF, DF, FSZ, SZ, CRM], If, Kr, Lv, Od, Sk, Vl, Vn, Zh.

Orthotrichum pállens Bruch ex Brid. - [CAM, MF, DF, FSZ, SZ, CRM], Ch, Ck, Cv, If, Ki, Km, Kr, Ky, Od, Po, Ri, Sk, Su, Te, Vl, Vn, Zh.

Orthotrichum pátens Bruch ex Brid. (O. stramineum var. patens Vent.) - [CAM, MF, DF, FSZ, SZ], Ck, Cv, If, Ke, Ky, Ri, Sk, Te, Vl, Vn, Zh.

Orthotrichum púmilum Sw. (O. fallax Bruch) - [CAM, MF, DF, FSZ, SZ, CRM], Ch, Ck, Cv, Dn, Do, If, Ka, Ke, Ki, Km, Kr, Ky, Lu, Lv, My, Od, Po, Ri, Sk, Su, Te, Vn, Vl.

Orthotrichum rupéstre Schleich. ex Schwaegr. - [CAM, FSZ, SZ, CRM], Ck, Dn, Do, If, Ki, Kr, Sk, Sp.

Orthotrichum scánicum Grönv. (O. leucomitrium Bruch \& Schimp.) - [DF], Cv.

Orthotrichum schímperi Hammar (O. pumilum Dicks.) - [CAM, MF, DF, FSZ, SZ, CRM], Cv, Do, If, Ke, Kr, Ky, Lv, Po, Sk, Te,Vn.

Orthotrichum speciósum Nees - [CAM, MF, DF, FSZ, SZ, CRM], Ch, Ck, Cv, Dn, Do, If, Ka, Ke, Ki, Km, Kr, Ky, Lu, Lv, My, Od, Po, Ri, Sk, Su, Te, Vl, Vn, Zh.

Orthotrichum stramíneum Hornsch. ex Brid. (O. rogeri var. defluens (Venturi) Venturi) - Cv, If, Km, Kr, Lv, Sk, Te.

Orthotrichum striátum Hedw. - [CAM, MF, DF, FSZ, SZ, CRM], Ch, Ck, Cv, If, Ka, $\mathrm{Ke}, \mathrm{Km}, \mathrm{Kr}, \mathrm{Ky}, \mathrm{Lv}, \mathrm{Po}, \mathrm{Ri}, \mathrm{Sk}, \mathrm{Su}, \mathrm{Te}, \mathrm{Vn}$.

Orthotrichum tenéllum Bruch ex Brid. - [CAM, FSZ, CRM], If, Kr, Po, Su.

Oxyrrhýnchium híans (Hedw.) Loeske (Eurhynchium hians (Hedw.) Sande Lac) [CAM, MF, DF, FSZ, SZ, CRM], Ch, Ck, Cv, Dn, Do, If, Ka, Ke, Ki, Km, Kr, Ky, Lu, Lv, My, Od, Po, Ri, Sk, Su, Te, Vn, Vl, Zh.

Oxyrrhynchium schléicheri (R. Hedw.) Röll (Eurhynchium schleicheri (R.Hedw.) Milde) - [CAM, CRM], Kr, Sk.

Oxyrrhynchium speciósum (Brid.) Warnst. (Eurhynchium speciosum (Brid.) Jur.) [CAM, MF, DF, SZ, CRM], Ck, Ke, Kr, Lv, Sk, Ri, Te.

Oxýstegus tenuiróstris (Hook. \& Taylor) Sm. (Trichostomum tenuirostre (Hook. \& Taylor) Lindb.) - [CAM, DF], Cv, If, Lv, Sk, Vn.

Palamocládium euchlóron (H. Müll.) Wijk \& Marg. - [CRM], Kr.

Paludélla squarrósa (Hedw.) Brid. - [MF, DF, FSZ], Ky, Lv, Ri, V1, Vn.

Palustriélla commutáta (Hedw.) Ochyra (Cratoneuron commutatum (Hedw.) G.Roth [CAM, MF, DF, CRM], Cv, If, Km, Kr, Lv, Sk.

Palustriella decípiens (De Not.) Ochyra (Cratoneuron decipiens (De Not.) Loeske) [CAM], Cv, If, Sk.

Palustriella falcáta (Brid.) Hedenäs (Cratoneuron falcatum (Brid.) G.Roth) - [CAM], Cv, If, Sk.

Paraleucóbryum enérve (Thed.) Loeske (Dicranum enerve Thed.) - [CAM], If, Sk.

Paraleucobryum longifólium (Hedw.) Loeske (Dicranum longifolium Ehrh.) - [CAM, MF, DF, FSZ], Ck, Cv, If, Ka, Km, Ky, Lv, Ri, Sk, Su, Vn, Zh.

Paraleucobryum sáutéri (Bruch \& Schimp.) Loeske (P. longifolium var. sauteri (Bruch \& Schimp.) C.E.O.Jensen) - [CAM], If, Sk.

Pelékium minútulum (Hedw.) Touw (Thuidium minutulum (Hedw.) Schimp.) - [MF, DF], Lv, Ri, Te, Zh, 
Pháscum pilíferum Hedw. (P. cuspidatum var. piliferum (Hedw.) Hook. \& Taylor, Tortula acaulon var. pilifera (With.) R.H. Zander) - [MF, DF, FSZ, SZ, CRM], Ch, Ck, $\mathrm{Cv}$, Do, If, Ke, Ki, Kr, Ky, Lu, Lv, My, Od, Po, Ri, Sp, Te, Vn.

Philonótis caespitósa Jur. - [CAM, MF], Vl, Sk, If, Ky, Ri, Cv.

Philonotis calcárea (Bruch \& Schimp.) Schimp.- [CAM, DF], Cv, If, Lv, Sk, Te.

Philonotis capilláris Lindb. (Ph. arnellii Husn., Ph. fontana subsp. capillaris (Lindb.) Herib.) - [CAM], Sk.

Philonotis fontána (Hedw.) Brid. - [CAM, MF, DF, FSZ, SZ], Ch, Cv, Do, If, Ka, Km, $\mathrm{Ky}, \mathrm{Lu}, \mathrm{Lv}, \mathrm{Sk}, \mathrm{Sp}, \mathrm{Te}, \mathrm{Vl}, \mathrm{Zh}$.

Philonotis márchica (Hedw.) Brid. - [CAM, MF, DF, FSZ], Cv, Sk, If, Km, Ky, Lv, Ri, Vn.

Philonotis seriáta Mitt. - [CAM], If, Sk.

Philonotis tomentélla Mol. (P. fontana var. pumila (Turner) Brid.) - [CAM], If, Sk.

Physcomitrélla pátens (Hedw.) Bruch \& Schimp - [CAM, MF, DF, FSZ, SZ], Ck, Dn, $\mathrm{Ka}, \mathrm{Ky}, \mathrm{Lv}, \mathrm{Te}$.

Physcomítrium arenícola Lazar. - [MF, FSZ, SZ], Ch, Dn, Do, Lu, My, Ke, Sp.

Physcomitrium eurystómum Sendt. (P. sphaericum var. eurystomum Husn. - [CAM, MF, DF, FSZ, SZ], Ch, Cv, Dn, Ky, Lu, Lv, Po, Sp.

Physcomitrium eurystómum Sendt. subsp. acuminátum (Bruch \& Schimp.) Giacom. (P. acuminatum Bruch \& Schimp.) - [CAM, DF], Cv, Km, Lv.

Physcomitrium pyrifórme (Hedw.) Bruch \& Schimp. - [CAM, MF, DF, FSZ, SZ], Ch, Ck, Cv, Dn, Do, If, Ka, Ke, Km, Ki, Kr, Ky, Lu, Lv, Po, Ri, Su, Te, Vl, Vn, Zh.

Physcomitrium sphaéricum (Ludw. ex Schkuhr) Brid. - [MF, DF], Ch. Km, Ky, Zh.

Plagiómnium affíne (Blandow ex Funck) T. Kop.- [CAM, MF, DF, FSZ, SZ, CRM], $\mathrm{Ch}, \mathrm{Ck}, \mathrm{Cv}$, Dn, If, Ka, Ke, Ki, Km, Kr, Ky, Lv, Po, Ri, Sk, Sp, Su, Vl, Vn, Zh.

Plagiomnium cuspidátum (Hedw.) T. Kop.- [CAM, MF, DF, FSZ, SZ, CRM], Ch, Ck, $\mathrm{Cv}$, Dn, Do, If, Ka, Ki, Km, Kr, Ky, Lu, Lv, My, Od, Po, Ri, Sk, Sp, Su, Te, Vl, Vn, Zh.

Plagiomnium elátum (Bruch \& Schimp.) T. Kop. (Mnium seligeri Jur. ex Warnst., M. affine var. elatum Bruch \& Schimp.) - [CAM, MF, DF, FSZ, CRM], Ch, Ck, Cv, If, Ka, $\mathrm{Km}, \mathrm{Kr}, \mathrm{Ky}, \mathrm{Lv}, \mathrm{Ri}, \mathrm{Sk}, \mathrm{Sp}, \mathrm{Vl}, \mathrm{Vn}, \mathrm{Zh}$.

Plagiomnium ellípticum (Brid.) T. Kop. (Mnium rugicum Laurer. emend Tuomik) [CAM, MF, DF, FSZ, CRM], - Ch, Ck, Cv, If, Ka, Ki, Km, Kr, Ky, Lv, Po, Ri, Sk, Su, Vl, Vn, Zh.

Plagiomnium médium (Bruch \& Schimp.) T. Kop. (Mnium medium Bruch \& Schimp.) [CAM, MF, FSZ, CRM], Ch, Ck, Cv, If, Ki, Ka, Kr, Ky, Lv, Po, Sk, Su, Vn.

Plagiomnium rostrátum (Schrad.) T. Kop. (Mnium rostratum Schrad., M. longirostre Brid.) - [CAM, MF, DF, FSZ, SZ, CRM], Ch, Ck, Cv, Dn, If, Ka, Km, Kr, Ky, Lv, Po, Ri, Sk, Su, Te, Vl, Vn, Zh.

Plagiomnium undulátum (Hedw.) T. Kop. (Mnium undulatum Hedw.) - [CAM, MF, DF, FSZ, SZ, CRM], Ch, Ck, Cv, Dn, If, Ka, Km, Kr, Ky, Lv, Po, Ri, Sk, Su, Te, Vl, Vn, Zh.

Plagiópus oederiánus (Sw.) Crum \& L.E.Anderson (P. oederi (Brid.) Limpr., Bartramia oederi Brid.) - [CAM, MF, DF, CRM], Cv, If, Kr, Lv, Sk, Zh.

Plagiothécium cavifólium (Brid.) Iwats. (P. roeseanum Schimp.) - [CAM, MF, DF, FSZ, $\mathrm{SZ}$, Ch, Ck, Cv, Dn, If, Ka, Ki, Ky, Lv, Ri, Sk, Su, Te, Vl, Vn, Zh.

Plagiothecium curvifólium Schlieph. ex Limpr. (P. laetum var. curvifolium (Schlieph. ex Limpr.) Mastracci \& M. Sauer) - [CAM, MF, DF, FSZ, CRM], Cv, If, Kr, Ky, Lv, Ri, Sk, $\mathrm{Su}, \mathrm{Vl}$.

Plagiothecium denticulátum (Hedw.) Schimp. - [CAM, MF, DF, FSZ, SZ], Ch, Ck, Cv, Dn, If, Ka, Ki, Ky, Lv, Po, Ri, Sk, Su, Te, Vl, Vn, Zh.

Plagiothecium denticulatum (Hedw.) Schimp. var. undulátum R.Ruthe ex Geh. (Plagiothecium ruthei Limpr.) - [CAM, MF], Cv, Ka, Ri, Su, Zh. 
Plagiothecium laétum Schimp. - [CAM, MF, DF, FSZ], Ch, Ck, Cv, If, Ka, Ky, Lv, Po, Ri, Sk, Su, Te, Vn, Vl, Zh.

Plagiothecium latebrícola Schimp. (Plagiotheciella latebricola (Wils.) Fleisch.) [CAM, MF, DF, FSZ], Ch, Ck, If, Ka, Lv, Po, Sk, Su, Vl, Zh.

Plagiothecium neckeroídeum Schimp. (P. noricum Molendo ex Limpr.) - [CAM], If, Sk.

Plagiothecium nemorále (Mitt.) Jaeg. (P. silvaticum (Brid.) Bruch \& Schimp., P. neglectum Mönk.) - [CAM, MF, DF, FSZ, CRM], Ch, Ck, Cv, If, Ka, Ki, Kr, Ky, Lv, Po, Sk, Sp, Su, Te, Vl,Vn.

Plagiothecium pilíferum (Sw.) Schimp. (Plagiotheciella pilifera (Sw.) Fleisch.) [CAM], Sk.

Plagiothecium platyphýllum Mönk. (Plagiothecium ruthei var. rupicola Limpr.) [CAM, DF, FSZ, CRM], Ch, Cv, If, Ka, Kr, Lv, Po, Sk, Su.

Plagiothecium succuléntum (Wils.) Lindb. - [CAM, MF, DF, FSZ, SZ], Ch, Ck, Cv, Dn, If, Ka, Ki, Km, Ky, Lv, My, Po, Ri, Sk, Su, Te, Vl, Vn, Zh.

Plagiothecium undulátum (Hedw.) Schimp. - [CAM, DF], Cv, If, Lv, Sk.

Plasteurhýnchium meridionále (Schimp.) Fleisch. (Eurhynchium meridionale (Schimp.) De Not., E. striatulum var. meridionale Schimp.) - [CAM, CRM], Cv, Kr.

Plasteurhynchium striátulum (Spruce) Fleisch. (Eurhynchium striatulum (Spruce) Schimp.) - [CAM, SZ, CRM], - Cv, If, Kr.

Platydíctya jungermannióides (Brid.) Crum (Amblystegium jungermannioides (Brid.) A.J.E.Sm., Amblystegiella sprucei (Brid.) Loeske) - [CAM, DF], Cv, If, Lv, Sk.

Platygýrium répens (Brid.) Schimp. - [CAM, MF, DF, FSZ, SZ], Ch, Ck, Cv, If, Ka, Ki, Km, Ky, Lu, Lv, My, Od, Po, Ri, Sk, Su, Te, Vl, Vn, Zh.

Pleurídium acuminátum Lindb. - [CAM, MF, DF, FSZ, SZ, CRM], Ch, Do, If, Kr, Ky, Lu, Lv, Ri, Sk, Su, Vn, Vl.

Pleuridium subulátum (Hedw.) Rabenh.- [CAM, MF, DF, FSZ, SZ], Cv, Dn, If, Lu, Lv, Ri, Sk, Su, Te, Vl, Vn.

Pleurózium schréberi (Willd. ex Brid.) Mitt. - [CAM, CRM], Ch, Ck, Cv, Dn, Do, If, $\mathrm{Ka}, \mathrm{Ki}, \mathrm{Km}, \mathrm{Kr}, \mathrm{Ky}, \mathrm{Lu}, \mathrm{Lv}, \mathrm{Po}, \mathrm{Ri}, \mathrm{Sk}, \mathrm{Su}, \mathrm{Te}, \mathrm{Vl}, \mathrm{Vn}, \mathrm{Zh}$.

Pogonátum aloídes (Hedw.) P. Beauv. - [CAM, MF, DF, FSZ, CRM], Ch, Cv, If, Kr, Lv, Sk, Vn, Zh.

Pogonatum nánum (Hedw.) P. Beauv. - [CAM, MF, CRM], If, Km, Kr, Sk,

Pogonatum urnígerum (Hedw.) P. Beauv. - [CAM, MF, DF, FSZ, CRM], Ch, Ck Cv, Dn, If, Ka, Kr, Ky, Lu, Lv, Po, Sk, Su, Te, Ka, Vn, Zh.

Póhlia andalúsica (Höhn.) Broth. - [MF, FSZ], Ch, Ck, Ky, Ri, Zh.

Pohlia annótina (Hedw.) Lindb. - [MF, FSZ, SZ], Ch, Ck, Ky, Mk, Ri, Su, Vn, Zh.

Pohlia bulbífera (Warnst.) Warnst. - [MF, FSZ], Ch, Ka, Ky, Ri, Su, Vl, Zh.

Pohlia camptotrachéla (Ren. \& Cardot) Broth. [CAM, MF], If, Ri.

Pohlia crúda (Hedw.) Lindb. - [CAM, MF, DF, FSZ, SZ, CRM], Ch, Ck, Cv, Dn, Do, If, Ka, Ki, Kr, Ky, Lv, My, Po, Ri, Sk, Sp, Su, Te, Vn, Zh.

Pohlia drúmmondii (H. Müll.) A.L.Andrews - [CAM], If.

Pohlia elongáta Hedw. (P. ambigua (Limpr.) Broth., P. acuminata Hornsch.) - [CAM, MF, DF], Cv, Sk, If, Lv, Zh.

Pohlia elongáta var. gréenii (Brid.) Shaw (Pohlia elongata var. minor Hartm., Pohlia minor Schleich ex Schwaegr.) - [CAM], If, Sk.

Pohlia fílum (Schimp.) Martensson (P. schleicheri H.A.Crum, P. gracilis (Schleich.) Lindb., P. rothii (Correns) Broth.) - [CAM], If.

Pohlia lescuriána (Sull.) Ochi (Mniobryum pulchellum (Hedw.) Loeske) - [CAM, FSZ], Sk, Te.

Pohlia longicólla (Hedw.) Lindb. (P. longicollis (Hedw.) Lindb.) - [CAM], Cv, If, Sk. 
Pohlia lúdwigii (Spreng. ex Schwaegr.) Broth. (Mniobryum ludwigii (Schwaegr.) Loeske). - [CAM], If.

Pohlia melanódon (Brid.) J. Shaw (P. delicatula (Hedw.) Grout, Mniobryum carneum (Schimp.) Limpr.) - [CAM, MF, DF, FSZ, CRM], Ch, Ck, Cv, If, Ka, Ke, Km, Kr, Ky, $\mathrm{Lv}, \mathrm{Sk}, \mathrm{Su}, \mathrm{Te}$.

Pohlia nútans (Hedw.) Lindb. - [CAM, MF, DF, FSZ, SZ, CRM], Ch, Ck, Cv, Dn, Do, If, Ka, Ke, Ki, Km, Kr, Ky, Lu, Lv, My, Od, Po, Ri, Sk, Sp, Su, Te, Vn, Vl, Zh.

Pohlia obtusifólia (Vill. ex Brid.) L. Koch [CAM], If, Sk.

Pohlia prolígera (Kindb.) Lindb. $-[\mathrm{MF}], \mathrm{Ch}$.

Pohlia wahlenbérgii (F.Weber \& Mohr) A.L.Andr. (Mniobryum wahlenbergii (F.Weber \& D. Mohr) Jenn., M. albicans Limpr.) - [CAM, DF, FSZ, SZ, CRM], Ch, Ck, Cv, If, $\mathrm{Ka}, \mathrm{Ki}, \mathrm{Km}, \mathrm{Kr}, \mathrm{Ky}, \mathrm{Lv}, \mathrm{My}, \mathrm{Po}$, Sk, Te, Vn.

Polytrichástrum alpínum (Hedw.) G. Sm. (Pogonatum alpinum (Hedw.) Rohl.) [CAM], Cv, If, Sk.

Polytrichastrum sexanguláre (Brid.) G. Sm. (Polytrichum sexangulare Hedw.) [CAM], If, Sk.

Polýtrichum commúne Hedw. - [CAM, MF, DF, FSZ, SZ], Ch, Ck, Cv, Dn, If, Ka, Ke, Km, Ky, Lu, Lv, My, Po, Ri, Sk, Su, Te, Vn, Vl, Zh.

Polytrichum formósum Hedw. (Polytrichastrum formosum Hedw G. Sm.) - [CAM, MF, DF, FSZ, SZ, CRM], Cv, Ch, Ck, Dn, If, Ka, Kr, Ky, Lv, Po, Ri, Sk, Su, Te, Vn, Vl, Zh.

Polytrichum juniperínum Hedw. - [CAM, MF, DF, FSZ, SZ, CRM], Ch, Ck, Cv, Dn, Do, If, Ka, Ke, Ki, Km, Kr, Ky, Lu, Lv, My, Od, Po, Ri, Sk, Sp, Su, Te, Vn, Vl, Zh.

Polytrichum longisétum Sw. ex Brid. (Polytrichastrum longisetum (Sw. ex Brid.) G. Sm., Polytrichum gracile Menz), - [CAM, MF, DF, FSZ, SZ], Ch, Ck, Cv, Dn, If, Ka, Ki, Km, Ky, Lu, Lv, Po, Ri, Sk, Su, Te, Vn, Vl, Zh.

Polytrichum pallidisétum Funck (Polytrichastrum pallidisetum (Funck) G. Sm., Polytrichum decipiens Limpr.) - [CAM, MF], Cv, If, Ri, Sk.

Polytrichum perigoniále Michx. (Polytrichum commune var. perigoniale (Michx.) Bruch \& Schimp.) - [CAM, MF, FSZ, SZ], Cv, If, Lv, My, Po, Ri, Sk, Su, Te, Vl.

Polytrichum pilíferum Hedw. - [CAM, MF, DF, FSZ, SZ, CRM], Ch, Ck, Cv, Dn, Do, If, Ka, Ke, Ki, Km, Kr, Ky, Lu, Lv, My, Od, Po, Ri, Sk, Sp, Su, Te, Vn, Vl, Zh.

Polytrichum stríctum Menz. ex Brid. (P. alpestre Hoppe) - [CAM, MF, DF, FSZ, SZ], $\mathrm{Ch}, \mathrm{Ck}, \mathrm{Cv}, \mathrm{Dn}, \mathrm{If}, \mathrm{Ka}, \mathrm{Ky}, \mathrm{Lu}, \mathrm{Lv}, \mathrm{Po}, \mathrm{Ri}, \mathrm{Sk}, \mathrm{Su}, \mathrm{Vn}, \mathrm{Vl}, \mathrm{Zh}$.

Polytrichum swártzii Hartm (P. uliginosum (Wallr.) Schriebl, P. commune var. swartzii (Hartm.) Nyholm) - [CAM], Cv, Sk.

Pseudephemérum nítidum (Hedw.) Loeske (Pleuridium nitidum (Hedw.) Rabenh.) [CAM, SZ], Sk, Sp.

Pseudoamblystégium súbtile (Hedw.) Vanderp. \& Hedenäs (Amblystegium subtile (Hedw.) Schimp., Platydictya subtilis (Hedw.) H.A.Crum, Amblystegiella subtilis (Hedw.) Loeske) - [CAM, MF, DF, FSZ, SZ, CRM], Ch, Ck, Cv, Dn, Do, If, Ka, Ke, Ki, Km, Kr, Ky, Lu, Lv, My, Od, Po, Ri, Sk, Sp, Su, Te, Vn, Vl, Zh.

Pseudóbryum cinclidióides (Huebener) T. Kop. (Mnium cinclidioides (Blitt) Hüb.) [CAM, MF], Cv, If, Sk, Zh.

Pseudocampýlium radicále (P. Beauv Vanderp. \& Hedenäs. (Amblystegium radicale ( $P$. Beauv.) Schimp., A. saxatile Schimp., Campylium radicale (P.Beauv.) Grout) - [CAM, MF, DF, FSZ, CRM], Ch, Cv, Dn, Ke, Kr, Ky, Lv, My, Po, Ri, Su, Vn, Zh.

Pseudocrossídium hornschuchiánum (Schultz) Zander (Barbula hornschuchiana Schultz) - [CAM, DF, FSZ, SZ, CRM], Cv, Ki, Kr, Lv, Te, Vn.

Pseudocrossidium revolútum (Brid.) Zander (Barbula revoluta Brid.) - [DF, FSZ, SZ, CRM], Kr, Lv, My, Od, Te, Vn. 
Pseudoleskeélla catenuláta (Brid. ex Schrad.) Kindb. (Pseudoleskea catenulata (Brid. ex Schrad.) B.S.G.) - [CAM, DF, FSZ, SZ, CRM], Do, If, Km, Kr, Lv, Sk, Te.

Pseudoleskeella nervósa (Brid.) Nyh. (Leskeella nervosa (Brid.) Loeske) - [CAM, MF, DF, FSZ, SZ, CRM], Ch, Ck, Cv, Dn, Do, If, Ka, Km, Ki, Kr, Ky, Lu, Lv, Od, Po, Ri, Sk, $\mathrm{Su}, \mathrm{Te}, \mathrm{Vl}, \mathrm{Vn}, \mathrm{Zh}$.

Pseudoleskeella rupéstris (Berggr.) Hedenäs \& L.Söderstr. (P. nervosa var. rupestris (Berggr.) Nyholm) - [CAM], Cv.

Pseudoleskeella tectórum (Funck ex Brid.) Kindb. ex Broth. - [CAM, DF, CRM], Cv, $\mathrm{Kr}$, Sk, Te.

Pseudoscleropódium púrum (Hedw.) Fleisch. (Scleropodium purum (Hedw.) Limpr.) [CAM, MF, DF, FSZ, SZ, CRM], Vl, Vn, Sk, If, Kr, Lv, Po, Ri, Su, Ch, Cv.

Pseudotaxiphýllum élegans (Brid.) Iwats. (Isopterygium elegans (Brid.) Lindb.) [CAM, DF, SZ], If, My, Sk, Te.

Pterygonéurum crossidióides W. Frey, Herrnst. \& Kürschner ( $P$. cavifolium var. crossidioides Sapeg.) - [SZ, CRM], Kr.

Pterygoneurum lamellátum (Lindb.) Jur. - [FSZ], Ck.

Pterygoneurum ovátum (Hedw.) Dix. (P. pusillum Broth.) - [CAM, MF, DF, FSZ, SZ, $\mathrm{CRM}$ ], Ch, Ck, Dn, Do, If, Ka, Ke, Ki, Kr, Ky, Lu, Lv, My, Od, Po, Ri, Sk, Su, Te, Vn, $\mathrm{Zh}$.

Pterygoneurum subséssile (Brid.) Jur.- [MF, DF, FSZ, SZ, CRM], Ch, Ck, Dn, Do, Ka, $\mathrm{Ke}, \mathrm{Ki}, \mathrm{Kr}, \mathrm{Ky}, \mathrm{Lu}, \mathrm{Lv}, \mathrm{My}, \mathrm{Od}, \mathrm{Po}, \mathrm{Sp}, \mathrm{Su}, \mathrm{Te}, \mathrm{Vn}$.

Pterigynándrum filifórme Hedw. - [CAM, MF, DF, FSZ, SZ, CRM], Ch, Ck, Cv, Dn, If, Ka, Ke, Kr, Lu, Lv, My, Od, Po, Sk, Su, Te, Vn, Zh.

Ptílium crista-castrénsis (Hedw.) De Not. - [CAM, MF, DF, FSZ, SZ], Ch, Cv, If, Ka, Km, Ky, Lv, Po, Sk, Su, Te, Vn, Vl, Zh.

Ptychostómum archangélicum Bruch \& Schimp. J.R. Spence (Bryum archangelicum Bruch \& Schimp., B. pendulum subsp. archangelicum (Bruch \& Schimp.) Lindb. - [CAM, MF, FSZ, SZ, CRM], Ch, Ck, Cv, Do, Kr, Lv, Sk.

Ptychostomum boreále (F.Weber \& D.Mohr) Ochyra \& Bednarek-Ochyra (Bryum pallescens Schleich. ex Schwaegr., B. cirratum Hoppe \& Horhsch.) - [CAM, MF, DF, FSZ, CRM], Ch, Ck, Cv, If, Ka, Ki, Km, Kr, Ky, Lu, Lv, Po, Ri, Sk, Su, Te, Vn.

Ptychostomum capilláre (Hedw.) Holyoak \& N.Pedersen (Bryum capillare Hedw.) [CAM, MF, DF, FSZ, SZ, CRM], Ch, Ck, Cv, Dn, Do, If, Ka, Ke, Ki, Km, Kr, Ky, Lv, My, Od, Po, Sk, Sp, Su, Te, Vl, Vn.

Ptychostomum cérnuum (Hedw.)Hornsch. (Bryum uliginosum (Brid.) Bruch \& Schimp. B. cernuum (Hedw.) Bruch \& Schimp.) - [CAM, MF, DF, FSZ], Cv, If, Lv, Sk, Su, Te.

Ptychostomum compáctum Hornsch (Bryum algovicum Sendt. ex H. Müll., B. compactum (Hornsch.) Kindb., B. pendulum (Hornsch.) Kind.), B. angustirete Kindb.) - [CAM, DF, FSZ, SZ, CRM], Ck, Cv, Do, Km, Kr, Lu, Lv, Sk, Te.

Ptychostomum crebérrímum (Taylor) J.R.Spence \& H.P.Ramsay (Bryum creberrimum Taylor) - [CAM, MF, DF, FSZ, SZ, CRM], Cv, Do, If, Ka, Ke, Kr, Lv, My, Po, Ri, Su, Vn.

Ptychostomum imbricátulum (Müll.Hal.) Holyoak \& N.Pedersen (B. amblyodon Müll. Hal., Bryum imbricatum (Schwaegr.) Bruch \& Schimp.) - [CAM, MF, FSZ, SZ, CRM], $\mathrm{Ch}, \mathrm{Ck}, \mathrm{Cv}, \mathrm{Do}, \mathrm{Kr}, \mathrm{Lv}, \mathrm{Sk}$.

Ptychostomum morávicum (Podp.) Ros \& Mazimpaka (Bryum moravicum Podp., B. capillare var. flaccidum Bruch \& Schimp. [CAM, FSZ, SZ, CRM], Cv, Do, Ka, Ke, Kr, Ky, My, Sk.

Ptychostomum pállens (Sw.) J.R.Spence (Bryum pallens Sw., B. subelegans Kindb., B. intermedium var. pallens (Sw.) Hartm., B. flaccidum Brid.) - [CAM, MF, DF, FSZ, SZ, $\mathrm{CRM}$, Ch, Ck, If, Ke, Km, Kr, Lv, My, Od, Sk, Sp, Su, Vl. 
Ptychostomum pseudotriquétrum (Hedw.) J.R.Spence \& H.P.Ramsay (Bryum pseudotriquetrum (Hedw.) P. Gaertn. et al.) - Ch, Cv, Do, If, Ka, Km, Kr, Lv, Sk, Su, Te, Vl, Zh.

Ptychostomum pseudotriquétrum (Hedw.) J.R.Spence \& H.P.Ramsay var. bímum (Schreb.) Holyoak \& N.Pedersen (Bryum bimum (Brid.) Turn. - [CAM, MF, DF, FSZ, $\mathrm{SZ}, \mathrm{CRM}]-\mathrm{Cv}$, Do, If, Ka, Kr, Ky, Lu, Lv, Ri, Sk, Vn.

Ptychostomum rúbens (Mitt.) Holyoak \& N.Pedersen (Bryum rubens Mitt.) - [CAM, MF, DF, FSZ, SZ], Ch, Cv, Do, Ke, Kr, Ky, Lv, My, Po, Sk, Su, Vl, Vn.

Ptychostomum torquéscens (Bruch \& Schimp.) Ros \& Mazimpaka (Bryum torquescens Bruch \& Schimp., B. capillare var. torquescens (Bruch \& Schimp.) Husn.) - [SZ, CRM], Do, $\mathrm{Kr}$.

Ptychostomum zíeri (Hedw.) Holyoak \& N.Pedersen (Plagiobryum zieri (Hedw.) Lindb.) - [CAM], If, Sk.

Pyláisia polyántha (Hedw.) Schimp. - [CAM, MF, DF, FSZ, SZ, CRM], Ch, Ck, Cv, Dn, Do, If, Ka, Ke, Ki, Km, Kr, Ky, Lu, Lv, My, Od, Po, Ri, Sk, Sp, Su, Te, Vn, Vl, Zh.

Pyramídula tetragóna (Brid.) Brid- [FSZ], Vn.

Racomítrium aciculáre (Hedw.) Brid. (Grimmia acicularis C.Muel.) - [CAM, MF], If, Sk, Zh.

Racomitrium affíne (F. Weber \& Mohr) Lindb. (R. heterostichum var. affine (F. Weber \& D. Mohr) Lesq.) - [CAM], If, Sk.

Racomitrium aquáticum (Brid. ex Schrad.) Brid. (R. protensum A.Braun) - [CAM], If, Sk.

Racomitrium canéscens (Hedw.) Brid. - [CAM, MF, DF, FSZ, CRM], Ch, Cv, If, Km, $\mathrm{Kr}, \mathrm{Ky}, \mathrm{Lv}, \mathrm{Po}, \mathrm{Ri}, \mathrm{Sk}, \mathrm{Su}, \mathrm{Te}, \mathrm{Vn}, \mathrm{Vl}, \mathrm{Zh}$.

Racomitrium elongátum Ehrh. ex Frisvoll (R. canescens var. intermedium Venturi \& Bott.) - [CAM, CRM], Kr, Sk.

Racomitrium ericóides (Brid.) Brid. (R. canescens var. ericoides (Brid.) Hampe) [CAM], If, Sk.

Racomitrium heteróstichum (Hedw.) Brid. - [CAM, MF, DF, CRM], If, Kr, Lv, Sk, Zh. Racomitrium lanuginósum (Hedw.) Brid. (R. hypnoides Lindb., Trichostomum lanuginosum Hedw.) - [CAM], If, Sk.

Racomitrium macóunii Kindb. - [CAM], If.

Racomitrium microcárpon (Hedw.) Brid.- [CAM], If, Lv, Sk.

Racomitrium sudéticum (Funck) Bruch \& Schimp. (R. heterostichum var. sudeticum (Funck) E.Bauer) - [CAM, CRM], If, Lv, Sk.

Rhabdowéisia crispáta (Dicks.) Lindb. (R. denticulata (Brid.) Bruch \& Schimp.) [CAM], Cv , If, Lv, Sk.

Rhabdoweisia fúgax (Hedw.) Bruch \& Schimp. (R. striata (Schrad.) Lindb.) - [CAM, MF, CRM], If, Kr, Sk, Zh.

Rhizómnium magnifólium (Horik.) T. Kop. (Mnium punctatum var. elatum Schimp.) [CAM, DF], Cv, If, Lv, Sk,

Rhizomnium pseudopunctátum (Bruch \& Schimp.) T. Kop. - [CAM, DF], Cv, If, Lv, Sk.

Rhizomnium punctátum (Hedw.) T. Kop. (Mnium punctatum Hedw.) - [CAM, MF, DF, FSZ, CRM], Ch, Ck, Cv, Dn, If, Ka, Ki, Km, Kr, Ky, Lv, Po, Sk, Sp, Su, Te, Vl, Vn, Zh.

Rhodóbryum ontariénse (Kindb.) Kindb. - [CAM, DF, FSZ, CRM], If, Kr, Sk, Te, Vn.

Rhodobryum róseum (Hedw.) Limpr. - [CAM, MF, DF, FSZ, SZ, CRM], Ch, Dn, If, $\mathrm{Ka}, \mathrm{Ki}, \mathrm{Km}, \mathrm{Ky}, \mathrm{Lv}, \mathrm{Po}, \mathrm{Ri}, \mathrm{Sk}, \mathrm{Su}, \mathrm{Vl}, \mathrm{Vn}, \mathrm{Zh}$.

Rhynchostegiélla tenélla (Dicks.) Limpr. - [DF, SZ, CRM], Do, Kr, Lv, Sp.

Rhynchostegiella teneríffae (Mont.) Dirkse \& Bouman - [CRM], Kr.

Rhynchostegium confértum (Dicks.) Schimp. - [DF], Lv. 
Rhynchostegium megapolitánum (Blandow ex F.Weber \& Mohr) Schimp. - [CAM, SZ, CRM], Kr, Lv, Sk.

Rhynchostegium murále (Hedw.) Schimp. - [CAM, MF, DF, SZ, CRM], Cv, If, Ke, $\mathrm{Km}, \mathrm{Kr}, \mathrm{Ky}, \mathrm{Lv}, \mathrm{Od}$, Sk, Te, Vn.

Rhynchostegium riparióides (Hedw.) Cardot (Platyhypnidium riparioides (Hedw.) Dix.) - [CAM, MF, DF, FSZ, SZ, CRM], Ck, Cv, If, Ki, Km, Kr, Ky, Lu, Lv, My, Ri, Sk, Su, $\mathrm{Vn}, \mathrm{Zh}$.

Rhynchostegium rotundifólium (Scop. ex Brid.) Schimp. - [CAM], Sk.

Rhytidiadelphus lóreus (Hedw.) Warnst. - [CAM], If, Sk.

Rhytidiadelphus squarrósus (Hedw.) Warnst. - [CAM, MF, DF, FSZ], Ch, Cv, If, Km, $\mathrm{Ky}, \mathrm{Lv}, \mathrm{Ri}, \mathrm{Sk}, \mathrm{Su}, \mathrm{Vl}, \mathrm{Vn}, \mathrm{Zh}$.

Rhytidiadelphus subpinnátus (Lindb.) T. Kop. (R. calvescens (Wils.) Broth.) - [CAM], $\mathrm{Cv}$, If, Sk.

Rhytidiadelphus triquétrus (Hedw.) Warnst. - [CAM, MF, DF, FSZ, SZ, CRM], Ch, Ck, Cv, Dn, Do, If, Ki, Km, Kr, Ky, Lv, Po, Ri, Sk, Su, Te, Vl, Vn, Zh.

Rhytídium rugósum (Hedw.) Kindb. - [CAM, DF, CRM], Cv, If, Kr, Sk, Te.

Saelánia glaucéscens (Hedw.) Broth.- [CAM, CRM], If, Kr, Sk.

Saniónia uncináta (Hedw.) Loeske (Drepanocladus uncinatus (Hedw.) Warnst.) [CAM, MF, DF, FSZ, SZ, CRM], Ch, Cv, If, Ka, Ke, Kr, Lv, Po, Ri, Sk, Su, Te, Vl,Vn.

Sarmentýpnum exannulátum (Schimp.) Hedenäs (Warnstorfia exannulata (Schimp.) Loeske, Drepanocladus exannulatus (Schimp.) Warnst.) - [CAM, MF, DF], Ch, Cv, If, Lv, Sk, Vl, Zt.

Sarmentypnum sarmentósum (Wahlenb.) Tuom. \& T.J. Kop. (Warnstorfia sarmentosa (Wahlenb.) Hedenäs, Calliergon sarmentosum (Wahlenb.) Kindb.) - [CAM, DF], Cv, Lv.

Schistídium agássizii Sull. \& Lesq. (Grimmia agassizii (Sull. Lesq.) A. Jaeger) - [CAM, CRM], Sk.

Schistídium apocárpum (Hedw.) Bruch \& Schimp. - [CAM, MF, DF, FSZ, SZ, CRM], $\mathrm{Ch}, \mathrm{Ck}, \mathrm{Cv}, \mathrm{Dn}$, Do, If, Ka, Ke, Ki, Km, Kr, Ky, Lu, Lv, My, Od, Po, Ri, Sk, Sp, Su, Te, Vn, Vl, Zh.

Schistídium brunnéscens Limpr.- [DF, SZ], Cv, Do, Km, Lv, My.

Schistídium crassípilum Blom - [CAM, CRM], Kr, Lv.

Schistídium duprétii (Thér.) W.A. Weber (S. apocarpum var. dupretii (Thér.) Wijk \& Margad.) - [CAM], If, Lv.

Schistídium elengátulum Blom - $[\mathrm{CRM}], \mathrm{Kr}$.

Schistídium fláccidum (De Not.) Ochyra (Grimmia flaccida (De Not.) Lindb.) - [DF, $\mathrm{CRM}], \mathrm{Kr}, \mathrm{Te}$.

Schistídium lancifólium (Kindb.) H.H. Blom [CAM, DF], Km, Lv.

Schistídium papillósum Culm. (S. apocarpum subsp. papillosum (Culm.) Poelt) [CAM, DF], Cv. If, Lv, Sk.

Schistídium púlchrum Blom - [CAM], If.

Schistídium rivuláre (Brid.) Podp. (S. apocarpum var. rivulare Bruch \& Schimp.) [CAM, MF, DF, CRM], If, Kr, Lv, Ri, Sk.

Schistídium robústum (Nees \& Hornsch.) Blom - [CAM], If.

Schistídium submúticum (Zickendr.) Blom - [MF, FSZ,], Vn, Zh.

Schistídium trichódon (Brid.) Poelt var. nútans Blom - [CAM], If.

Schistostéga pennáta (Hedw.) F.Weber \& Mohr - [CAM, MF, SZ], Cv, Dn, If, Sk, Zt.

Sciuro-hýpnum cúrtum (Lindb.) Ignatov (Brachythecium curtum (Lindb.) Limpr.) [MF, DF], If, Ky,Lv, Ri, Te, Vn, Zh.

Sciuro-hypnum flotowiánum (Sendt.) Ignatov \& Huttunen (Cirriphyllum reichenbachianum (Huebener) Wijk \& Margad., Eurhynchium flotowianum (Sendtn.) Kartt.) - [CAM,CRM], If, Kr, Lv, Sk. 
Sciuro-hypnum plumósum (Hedw.) Ignatov \& Huttunen (Brachythecium plumosum (Hedw.) Schimp.) - [CAM, MF, DF], Cv, If, Ka, Lv, Sk, Te, Zh.

Sciuro-hypnum popúleum (Hedw.) Ignatov \& Huttunen (Brachythecium populeum (Hedw.) Schimp.) - [CAM, MF, DF, FSZ, SZ, CRM], Ch, Ck, Cv, Dn, If, Ka, Km, Kr, Ky, Lu, Lv, Od, Po, Ri, Sk, Te, Vn, Zh.

Sciuro-hypnum refléxum (Starke) Ignatov \& Huttunen (Brachythecium reflexum (Starke) Schimp.) - [CAM, MF, DF, FSZ], If, Km, Lv, Od, Po, Sk, Su, Te, Zh.

Sciuro-hypnum stárkei (Brid.) Ignatov \& Huttunen (Brachythecium starkei (Brid.) Schimp.) - [CAM], If, Ke, Kr, Lv.

Scorpídium cossónii (Schimp.) Hedenäs (Drepanocladus intermedius (Lindb.) Warnst., D. cossonii (Schimp.) Loeske) - [MF], Ri, Vl.

Scorpidium revólvens (Sw.) Rubers (Drepanocladus revolvens (Sw..) Warnst.) - [CAM, MF, DF, FSZ], If, Km, Lv, Ri, Sk, Vl.

Scorpidium scorpióides (Hedw.) Limpr. - [MF, DF], Lv, Ri, V1.

Scorpiúrium circinátum (Bruch) Fleisch. \& Loeske (Eurhynchium circinatum (Brid.) Bruch \& Schimp.) - [SZ, CRM], Kr.

Seligéria brevifólia (Lindb.) Lindb. (S. pusilla var. brevifolia Lindb.) - [CAM], If, Sk.

Seligeria calcárea (Hedw.) Bruch \& Schimp. - [CAM, MF, DF, FSZ, SZ], Ch, Do, If, $\mathrm{Ka}, \mathrm{Lu}, \mathrm{Lv}, \mathrm{Sk}$.

Seligeria campylopóda Kindb. - [CAM, DF], If, Lv, Sk.

Seligeria donniána (Sm.) H. Müll. - [CAM, DF], If, Lv, Km, Te, Sk.

Seligeria pusílla (Hedw.) Bruch \& Schimp. - [CAM, DF, CRM], Cv, If, Kr, Lv, Sk, Te.

Seligeria recurváta (Hedw.) Bruch \& Schimp. - [CAM, DF, CRM], Cv, If, Km, Kr, Lv, $\mathrm{Sk}, \mathrm{Te}$.

Serpoléskea confervóides (Brid.) Loeske (Amblystegium confervóides (Brid.) Schimp., Platydictya confervoides (Brid.) H.A.Crum, Amblystegiella confervoides (Brid.) Loeske) [CAM, DF, FSZ, SZ], Cv, If, Ki, Km, Lv, Po, Ri, Sk, Te.

Sphágnum angustifólium (C. Jensen ex Russow) C. Jensen - [CAM, MF], Ch, If, Ka, $\mathrm{Ky}, \mathrm{Ri}, \mathrm{Sk}, \mathrm{Vl}, \mathrm{Zh}$.

Sphagnum auriculátum Schimp. (S. denticulatum Brid.) - [CAM, MF], Ch, Ka, Ri, Vl, Zh.

Sphagnum bálticum (Russow) C. Jensen (S. recurvum subsp. balticum Russ.) - [CAM], Sk.

Sphagnum capillifólium (Ehrh.) Hedw. (S. nemoreum Scop., S. acutifolium Ehrh.) [CAM, MF,DF, FSZ, SZ], Ch, Cv, Dn, Do, If, Ka, Km, Ky, Lv, Po, Ri, Sk, Su, Vl,Vn, Zh. Sphagnum centrále C. Jensen - [CAM, MF, DF, FSZ, SZ], Ch, Cv, Dn, If, Ka, Km, Ky, Lu, Lv, Sk, Su, Po, Ri, Vl, Vn.

Sphagnum compáctum Lam. \& DC. - [CAM, MF, FSZ], Ch, Ck, Ka, Ky, Lu, Lv, Po, $\mathrm{Ri}$, Sk, Su, Vl, Vn, Zh.

Sphagnum contórtum Schultz - [CAM, MF, DF, FSZ, SZ], Ch, Sk, If, Ka, Km, Ky, Po, Vl, Zh.

Sphagnum cuspidátum Ehrh. ex Hoffm. - [CAM, MF, DF, FSZ, SZ], Ch, If, Ka, Ky, Lv, Ri, Sk, Su, Vl, Zh.

Sphagnum fállax (Klinggr.) Klinggr. (S. recurvum var. recurvum P. Beauv., S. recurvum var.mucronatum (Russ.) Warnst.) - [CAM, MF, DF, FSZ, SZ], Ch, Ck. Dn, Do, If, Ka, $\mathrm{Ke}, \mathrm{Km}, \mathrm{Ky}, \mathrm{Lu}, \mathrm{Lv}, \mathrm{Po}, \mathrm{Ri}, \mathrm{Sk}, \mathrm{Su}, \mathrm{Vl}, \mathrm{Vn}, \mathrm{Zh}$.

Sphagnum fimbriátum Wils. - [CAM, MF, DF, FSZ, SZ], Ch, Ck. Dn, Do, Ka, Ke, Ky, Lu, Lv, Po, Ri, Sk, Su, Vl,Vn, Zh.

Sphagnum flexuósum Dozy \& Molk. (S. recurvum var. amblyphyllum (Russ.) Warnst.). - [CAM, MF, DF, FSZ, SZ], Ch, Ck, Dn, Do, Sk, Ka, Km, Ki, Ky, Lu, Lv, Po, Ri, Su, $\mathrm{Vl}, \mathrm{Vn}, \mathrm{Zh}$. 
Sphagnum fúscum (Schimp.) Klinggr. - [CAM, MF, DF, SZ], Ch, Do, If, Ky, Lv, Ri, Sk, Vl, Zh.

Sphagnum girgensóhnii Russow (S. fimbriatum var. strictum Lindb.) - [CAM, MF, FSZ], Ch. If, Ka, Ky, Lv, Ri, Sk, Su, Vl, Zh.

Sphagnum inundátum Russow (S. subsecundum var. inundatum (Russow) C. Jensen) [CAM, MF], Ka, Ri, Sk.

Sphagnum magellánicum Brid. - [CAM, MF, DF, FSZ, SZ], Ch, Ck. Dn, If, Ka, Km, $\mathrm{Ky}, \mathrm{Lv}, \mathrm{Po}, \mathrm{Ri}, \mathrm{Sk}, \mathrm{Su}, \mathrm{Vl}, \mathrm{Vn}, \mathrm{Zh}$.

Sphagnum május (Russow) C. Jensen (S. dusenii C.Jens.) - [CAM, MF, DF, FSZ], Ch, Ck, If, Ka, Ky, Ri, Sk, Vl, Zh.

Sphagnum mólle Sull. ( S. tabulare Sull.) - [MF, DF], Lv, Zh.

Sphagnum obtúsum Warnst. (S. recurvum var.obtusum Warnst.) - [CAM, MF, DF, FSZ, SZ], Ch, Ck, Dn, Do, If, Ka, Ky, Lv, Po, Ri, Sk, Vl,Vn, Zh.

Sphagnum palústre L. - [CAM, MF, DF, FSZ, SZ],Ch, Ck, Cv, Dn, Do, If, Ka, Km, Ky, Lu, Lv, Po, Ri, Sk, Su, Te, Vl,Vn, Zh.

Sphagnum papillósum Lindb. - [CAM, MF, DF, FSZ, SZ],Ch, Do, Ka, Ky, Ri, Sk, Vl, $\mathrm{Zh}$.

Sphagnum platyphýllum (Lindb. ex Brait.hw.) Warnst - [CAM, MF, DF, FSZ],Ch, Cv, If, Km, Ky, Ri, Sk, Su, Vl,Vn, Zh.

Sphagnum quinquefárium (Braithw.) Warnst. - [CAM, MF, DF], Cv, If, Lv, Sk.

Sphagnum ripárium Ångstr. - [CAM, DF], If, Ri, Sk, Vl.

Sphagnum rubéllum Wils. - [FZ], If, Ri, Sk, Vl, Zh.

Sphagnum rússowii Warnst. (S. robustum (Russ.) Röl.) - [CAM, MF, DF],Ch. If, Ky, Lv, $\mathrm{Sk}, \mathrm{Su}, \mathrm{Vl}, \mathrm{Zh}$.

Sphagnum squarrósum Crome - [CAM, MF, DF, FSZ, SZ], Ch, Ck, Cv, Dn, If, Ka, Ke, $\mathrm{Ki}, \mathrm{Ky}, \mathrm{Lu}, \mathrm{Lv}, \mathrm{Po}, \mathrm{Ri}, \mathrm{Sk}, \mathrm{Sp}, \mathrm{Su}, \mathrm{Vl}, \mathrm{Vn}, \mathrm{Zh}$.

Sphagnum subnítens Russow \& Warnst. (S. plumulosum Röll) - [CAM, DF],Cv, Sk, Zh. Sphagnum subsecúndum Nees - [CAM, MF, DF, FSZ, SZ], Ch, Ck, Cv, Dn, If, Ka, Ky, Lv, Po, Ri, Sk, Su, Vl,Vn, Zh.

Sphagnum tenéllum (Brid.) Pers. ex Brid. - [CAM, DF] ,Sk, Zh.

Sphagnum téres (Schimp.) Ångstr. - [CAM, MF, DF, FSZ, SZ], Ch, Ck, If, Ka, Ki, Ky, Lv, Po, Ri, Sk, Su, Vl, Zh.

Sphagnum warnstórfii Russow - [CAM, MF, DF, SZ],Ch, Dn, If, Ky, Lv, Ri, Sk, Su, Vl, Zh.

Sphagnum wulfiánum Girg. - [DF], Ri, Zh.

Spláchnum ampulláceum Hedw. - [CAM, MF, DF], Ch, If, Ky, Lv, Sk, Vl, Zh.

Splachnum sphaéricum Hedw. - [CAM, MF], If, Sk, Vl.

Straminérgon stramíneum (Dicks. ex Brid.) Hedenäs (Calliergon stramineum (Dicks. ex Brid.) Kindb.) - [CAM, MF, DF, FSZ], Ch, If, Ky, Lv, Po, Ri, Sk, Su, Vl, Zh.

Syntríchia calcícola J.J. Amann (Syntrichia ruralis var. calcicola Malta, Tortula calcicolens W.A.Kramer) - [CAM, DF, FSZ, SZ, CRM], Ck, Cv, Do, If, Ki, Kr, Lu, Ri, Sk, Te, Vn.

Syntrichia caninérvis Mitt. (S. desertorum Broth., Tortula caninervis (Mitt.) Broth.) [SZ, CRM], Dn, Do, Ke, Kr, Lu, Od.

Syntrichia hándelii (Schiffn.) S. Agnew \& Vondr. (Tortula handelii Schiffn,) - [SZ],

Do, Lu.

Syntrichia laevipíla Brid. - [CRM], Kr.

Syntrichia latifólia (Bruch ex Hartm.) Huebener (Tortula latifolia Bruch ex Hartm.) [CAM, FSZ], Ka, Sk.

Syntrichia montána Nees (Tortula montana Lindb.) - [CAM, MF, DF, FSZ, SZ, CRM], $\mathrm{Ch}, \mathrm{Ck}, \mathrm{Cv}$. Dn, Do, If, Ke, Km, Kr, Lv, My, Ri, Sk, Su, Vn. 
Syntrichia norvégica F. Weber (Tortula norvegica (F.Weber) Lindb.) - [CAM, FSZ, CRM], Kr, Sk, Vn.

Syntrichia papillósa (Wils.) Jur. (Tortula papillosa Wilson) - [CAM, MF, DF, FSZ, SZ], $\mathrm{Ck}, \mathrm{Cv}$ If, Ke, Lv, Sk, Vl, Vn.

Syntrichia ruralifórmis (Besch.) Cardot (S. ruralis var. arenicola J.J.Amann, Tortula ruralis var. ruraliformis (Besch.) Delogne, Tortula ruraliformis (Besch.) Cardot) [CAM, MF, DF, FSZ, SZ, CRM], Vl, Dn, Do, Sk, Sp, Ky, Kr, Lu, My, Od, Po, Ri, Su, Te, $\mathrm{Ke}, \mathrm{Km}, \mathrm{Ch}, \mathrm{Cv}$.

Syntrichia rurális (Hedw.) F. Weber \& Mohr (Tortula ruralis (Hedw.) P.Gaertn., B.Mey. \& Scherb.) - [CAM, MF, DF, FSZ, SZ, CRM], Ch, Ck, Cv, Dn, Do, If, Ka, Ke, Ki, Km, $\mathrm{Kr}, \mathrm{Ky}, \mathrm{Lu}, \mathrm{Lv}, \mathrm{My}, \mathrm{Od}, \mathrm{Po}, \mathrm{Ri}, \mathrm{Sk}, \mathrm{Sp}, \mathrm{Su}, \mathrm{Te}, \mathrm{Vn}, \mathrm{Vl}, \mathrm{Zh}$.

Syntrichia rurális (Hedw.) F. Weber \& Mohr var. polysporogónica Boiko [SZ], Ke, My. Syntrichia sinénsis (H. Müll.) Ochyra (Tortula sinensis (Müll.Hal.) Broth.) - [CAM, CRM], Kr, Sp.

Syntrichia víréscens (De Not.) Ochyra (S. pulvinata (Jur.) Jur, Tortula virescens (De Not.) De Not.) - [CAM, MF, DF, FSZ, SZ, CRM], Ch, Do, If, Ke, Ki, Km, Kr, Lu, Lv, Od, Po, Ri, Sk, Vl, Vn, Zh.

Taxiphýllum wissgríllii (Garov.) Wijk \& Marg. (T. depressum (Bruch) Reim) - [CAM, MF, DF, FSZ, SZ, CRM], Ck, Cv, Dn, If, Kr, Ri, Sk, Te, Vn, Zh.

Taylória linguláta (Dicks.) Lindb. - [CAM], If.

Tayloria serráta (Hedw.) Bruch \& Schimp. - [CAM], If, Sk.

Tayloria ténuis (Dicks.) Schimp. (T. serrata var. tenuis (Dicks.) Bruch \& Schimp.) [CAM], If, Sk.

Tetráphis pellúcida Hedw. - [CAM, MF, DF, FSZ], Ch, Ck, Cv, If, Ka, Km, Ky, Lv, Ri, Sk, Su, Te, Vn, Vl, Zh.

Tetráplodon angustátus (Hedw.) Bruch \& Schimp. - [CAM], If, Sk,

Tetraplodon mnióides (Hedw.) Bruch \& Schimp. - [CAM], If.

Tetrodóntium browniánum (Dicks.) Schwaegr. - [CAM], Cv, If, Sk.

Tetrodontium ovátum (Funck) Schwaegr - [CAM], If.

Thamnóbryum alopécurum (Hedw.) Gangulee (Thamnium alopecurum (Hedw.) Bruch \& Schimp.) - [CAM, MF, DF, FSZ, CRM], Cv, If, Kr, Lv, Sk, Vn.

Thuídium assímile (Mitt.) Jaeg. (T. philibertii Limpr.) - [CAM, MF, DF, FSZ, SZ, CRM], Ch, Ck, Cv, Dn, Do, If, Ka, Km, Kr, Ky, Lv, Po, Ri, Sk, Su, Te, Vl, Vn, Zh.

Thuidium delicátulum (Hedw.) Schimp. - [CAM, MF, DF, FSZ], Ch, Ck, Cv, If, Ka, Km, Ky, Lv, Po, Ri, Sk, Su, Te, Vl, Vn, Zh.

Thuidium recógnitum (Hedw.) Lindb. - [CAM, MF, DF, FSZ, SZ, CRM], Ch, Cv, Do, If, Ka, Km, Kr, Ky, Lv, Po, Ri, Sk, Te, Vl, Vn, Zh.

Thuidium tamariscínum (Hedw.) Schimp. - [CAM, MF, DF, FSZ, CRM], Ch, Cv, If, $\mathrm{Km}, \mathrm{Kr}, \mathrm{Lv}, \mathrm{Sk}, \mathrm{Su}, \mathrm{Vl}, \mathrm{Zh}$.

Tímmia austríaca Hedw. - [CAM, FSZ], Sk, Te.

Timmia bavárica Hessl. - [CAM, DF, FSZ, CRM], If, Kr, Lv, Sk, Te.

Timmia megapolitána Hedw. - [CAM, MF, DF, FSZ], Ch, If, Lv, Vn.

Tomenthýpnum nítens (Hedw.) Loeske (Camptothecium nitens Schimp.) - [CAM, MF, DF, FSZ], Ch, Cv, If, Ky, Lv, Ri, Sk, Te, Vl, Vn, Zh.

Tortélla frágilis (Hook. \& Wils.) Limpr. - [CRM], Kr.

Tortella húmilis (Hedw.) Jenn. (T. caespitosa Limpr.) - [CAM, SZ, CRM], Cv, Do, If, $\mathrm{Kr}$, Sk.

Tortella inclináta (R. Hedw.) Limpr. - [CAM, DF, SZ, CRM], Cv, If, Ka, Ke, Kr, Lv, Sk.

Tortella squarrósa (Brid.) Limpr. (Pleurochaete squarrosa (Brid.) Lindb.) - [CRM], Kr. 
Tortella tortuósa (Hedw.) Limpr. - [CAM, DF, FSZ, SZ, CRM], Ck, Cv, Dn, If, Ki, Kr, Lu, My, Ri, Sk, Te, Vn.

Tórtula acáulon With. R.H.Zander (Phascum cuspidatum Hedw., Phascum acaulon Lindb.) - [CAM, MF, DF, FSZ, SZ, CRM], Ch, Ck, Cv, Dn, Do, If, Ka, Ke, Ki, Km, Kr, Ky, Lu, Lv, My, Od, Po, Ri, Sk, Te, Vn.

Tortula aestíva (Schultz) P. Beauv. (T. muralis var. aestiva Hedw.) - [CAM, MF, DF, FSZ, SZ], Ch, Cv, Dn, Do, If, Ka, Ke, Ki, Km, Ky, Lu, Lv, My, Od, Sk, Te, Vn.

Tortula canéscens Mont. - [CAM, DF, SZ, CRM], Kr, Sk, Te.

Tortula caucásica Lindb. ex Broth. (Tortula modica Zander, Pottia intermedia (Turner) Fürnr.) - [CAM, MF, DF, FSZ, SZ, CRM], Ch, Ck, Cv, Dn, Do, If, Ka, Ke, Kr, Ky, Lu, Lv, Po, Ri, Sk, Su, Te, Vn, Zh.

Tortula cérnua (Huebener) Lindb. (Desmatodon cernuus (Huebener) Bruch \& Schimp.) - [FSZ], Ch, Ck, Ky.

Tortula cuneifólia (Dicks.) Turn. - [FSZ], Su.

Tortula hoppeána (Schultz) Ochyra (Desmatodon latifolius (Hedw.) Brid.) - [CAM, DF, SZ], Do, If, Lv, Sk, Te.

Tortula inérmis (Brid.) Mont. (Syntrichia inermis (Brid.) Bruch) - [CRM], Kr.

Tortula leucostóma (R.Br.) Hook. \& Grev. (Desmatodon leucostomus (R. Br.) Berggr.) [DF], Te.

Tortula lindbérgii Kindb. ex Broth. (Pottia lanceolata (Hedw.) Müll. Hal., Tortula lanceola R. H. Zander.) - [CAM, DF, FSZ, SZ, CRM], Cv, Dn, Do, If, Ke, Ki, Km, Kr, $\mathrm{Ky}, \mathrm{Lu}, \mathrm{Lv}, \mathrm{My}, \mathrm{Od}, \mathrm{Sk}, \mathrm{Sp}, \mathrm{Te}, \mathrm{Vn}$.

Tortula linguláta Lindb. $-[\mathrm{DF}], \mathrm{Lv}$.

Tortula mucronifólia Schwaegr. (Syntrichia mucronifolia (Schwägr.) Brid.) - [MF, FSZ, $\mathrm{SZ}, \mathrm{CRM}]$, Do, Ka Ki, Kr, Lv, Ri, Su, Te, Vn.

Tortula murális Hedw. - [CAM, MF, DF, FSZ, SZ, CRM], Ch, Ck, Cv, Dn, Do, If, Ka, $\mathrm{Ke}, \mathrm{Ki}, \mathrm{Km}, \mathrm{Kr}, \mathrm{Ky}, \mathrm{Lu}, \mathrm{Lv}, \mathrm{My}, \mathrm{Od}, \mathrm{Po}, \mathrm{Ri}, \mathrm{Sk}, \mathrm{Sp}, \mathrm{Su}, \mathrm{Te}, \mathrm{Vn}, \mathrm{Vl}, \mathrm{Zh}$.

Tortula protobryóides R.H. Zander (Protobryum bryoides (Dicks.) J.Guerra \& M.J.Cano, Pottia bryoides (Dicks.) Mitt., Mildeella bryoides Limpr.) - [CAM, DF, FSZ, $\mathrm{SZ}, \mathrm{CRM}$ ], Ck, Cv, Dn, Do, If, Ka, Ke, Ki, Kr, Ky, Lu, Lv, My, Od, Po, Sp, Su, Te, Vn.

Tortula rándii (Kenn.) Zander (Desmatodon randii (Kenn.) Laz., Pottia randii Kenn.) [FSZ], Ch, Ck, Po.

Tortula schímperi M.J.Cano, O.Werner \& J.Guerra (T. subulata var. angustata (Schimp.) Limpr.) - [CAM, CRM], Cv, If, Kr, Sk.

Tortula subuláta Hedw. (Syntrichia subulata (Hedw.) Web. \& Mohr) - [CAM, MF, DF, FSZ, SZ, CRM], Ch, Dn, Do, If, Ka, Ke, Ki, Km, Kr, Ky, Lv, My, Po, Ri, Sk, Su, Te, Vn, $\mathrm{Zh}$.

Tortula truncáta (Hedw.) Mitt. (Pottia truncata (Hedw.) Bruch \& Schimp., P. truncatula Warnst.) - [CAM, MF, DF, FSZ, SZ, CRM], Ch, Ck, Dn, If, Ka, Ke, Ki, Km, Kr, Ky, Lu, Lv, My, Po, Ri, Sk, Su, Te, Vn, Zh,

Tortula ucraínica (Lazar.) Zander (Desmatodon ucrainicus Laz.) - [FSZ], Ck.

Tremátodon ambíguus (Hedw.) Hornsch. - [MF], Ky, Ri.

Tríchodon cylíndricus (Hedw.) Schimp. (Ditrichum cylindricum (Hedw.) Grout) [CAM, MF, DF, FSZ, SZ], Ck, Dn, If, Ka, Ki, Ky, Lv, Sk, Su,Vn.

Trichostómum brachydóntium Bruch (T. mutabile Bruch) - [CAM, CRM], If, Kr. Sk, Trichostomum críspulum Bruch (Trichostomum brevifolium Sendt., Trichostomum viridulum Bruch) - [CAM, DF, CRM], Cv, If, Ka, Kr, Lv, Sk Te.

Ulóta brúchii Hornsch. ex Brid. (U. crispa var. norvegica (Gronvall) A.J.E.Sm. \& M.O.Hill) - [CAM], Cv, If, Lv, Sk.

Ulota coarctáta (P. Beauv.) Hammar (U. ludwigii Brid.) - [CAM, MF], Cv, If, Lv, Sk, $\mathrm{Zh}$. 
Ulota críspa (Hedw.) Brid. - [CAM, MF, DF, FSZ, CRM], Ch, Ck, Cv, If, Ka, Ke, Km, $\mathrm{Kr}, \mathrm{Ky}, \mathrm{Lv}, \mathrm{Ri}, \mathrm{Sk}, \mathrm{Te}, \mathrm{Vl}, \mathrm{Vn}, \mathrm{Zh}$.

Ulota drúmmondii (Hook. \& Grev.) Brid. - [CAM], If.

Ulota hútchinsiae (Sm.) Hammar (U. americana (P. Beauv.) Limpr.) - [CAM], Cv, If, Sk.

Ulota rehmánnii Jur. - [CAM], If.

Warnstórfia flúitans (Hedw.) Loeske (Drepanocladus fluitans (Hedw.) Warnst.) [CAM, MF, DF, FSZ, SZ], Ch, Ck, If, Ka, Ky, Lv, Od, Po, Ri, Sk, Su, Vl, Vn, Zh.

Warnstorfia pseudostramínea (Müll. Hal.) Tuom. \& T.J. Kop. (Drepanocladus fluitans (Hedw.) Warnst.f. pseudostraminea (C. Müll.)Mönk.) [CAM, MF].

Weíssia brachycárpa (Nees \& Hornsch.) Jur. (W. microstoma (Hedw.) C. Müll..) [CAM, MF, DF, FSZ, SZ, CRM], Ch, Ck, Cv, Do, If, Ka, Kr, Ky, Lv, Po, Ri, Sk, Su, Te, $\mathrm{Vl}, \mathrm{Vn}$.

Weissia condénsa (Voit) Lindb. (W. tortilis (Schwagr.) C. Müll.) - [CAM, MF, DF, FSZ, $\mathrm{SZ}, \mathrm{CRM}$ ], Ch, Ck, Dn, Do, If, Ka, Ki, Km, Kr, Lu, Lv, My, Sk.

Weissia controvérsa Hedw. (W. fallax Sehlm., W. viridula Hedw.) - [CAM, DF, FSZ, $\mathrm{CRM}$ ], Ck, Cv, If, Kr, Lv, Po, Sk, Su, Te, Vn.

Weissia controvérsa var. crispáta Nees \& Hornsch.) Nyholm (Hymenostomum crispatum Nees et Hornsch., W. fallax Sehlm.) - [DF, CRM], Lv, Kr.

Weissia levíeri (Limpr.) Kindb. (Astomum crispum var. levieri Mönk., A. crispum var. phylibertii (Husn.) Wijk \& Marg.) - [SZ, CRM], Do, Ka, Ke, Kr, Lu.

Weissia longifólia Mitt. (Astomum crispum (Hedw.) Hampe) - [CAM, MF, DF, FSZ,SZ,CRM], Ch, Ck, Cv, Dn, Do, If, Ka, Ke, Km, Ki, Kr, Ky, Lu, My, Od, Po, Ri, Sk, $\mathrm{Sp}, \mathrm{Su}, \mathrm{Te}, \mathrm{Vn}$.

Weissia rostelláta (Brid.) Lindb. $-[\mathrm{CAM}]$, Sk.

Weissia rútilans (Hedw.) Lindb. - [CAM, DF], Sk, Te.

Zýgodon dentátus (Limpr.) Kart. - [CAM], If.

Zygodon rupéstris Schimp. ex Lorentz (Z. viridissimus var. rupestris C. Hartm.) [CAM, CRM], Kr, Sk.

Zygodon viridíssimus (Dicks.) Brid. - [CRM], Kr.

\section{New to Ukrainian bryoflora and nomenclature changed taxa compared to The Checklist of bryophytes of Ukraine (2008)}

During the last period The List of Bryophytes in Ukraine was replenished with a large number of species and varieties. It takes into account a number of taxa, which for various reasons were not included in Checklist of 2008 [BOIKO, 2008]. There have been significant changes in the nomenclature of bryophyte taxa while processing the results of the latest research. Species of several families that have been presented in the Checklist of bryophytes 2008, due to taxonomic changes are considered as of other genera. These types of families are Pleurochaete, Platyhypnidium, Protobryum, Pseudocalliergon, Pseudoleskea, Pterogonium, Ptychodium. Instead, the new Checklist compared to Checklist of 2008, new families have appeared. This is due to new discoveries, new descriptions and nomenclature changes besides some taxa of the Checklist of 2008 were as synonyms. From Marchantiophyta division Asterella, Clevea, Endogemma, Isopaches, Leiomylia, Liochlaena, Lophoziopsis, Mesoptychia, Neoorthocaulis, Schistochilopsis, Schljacovianthus, Sphenolobus, Syzygiella, Trilophozia, Bryophyta division - Campylidium, Exsertotheca, Hymenoloma, Imbribryum, Lazarenkia, Myrinia, Nogopterium, Nyholmiella, Pseudoamblystegium, Pseudocampylium, Ptychostomum, Sarmentypnum, Serpoleskea. These materials are taken from briologists' works [ZANDER, 1993; BLOM, 1996; WERNER et al., 2002, 2004, 2005, 2013; OCHYRA et al., 2003; GOFFINET et al., 2004; SZWEYKOWSKI et al., 2005; VIRCHENKO, 2005; GRUNDMANN et 
al., 2006; Hedenas, 2006; Danilkiv et al., 2007; Holyoak, Pedersen, 2007; Ignatov, Milyutina, 2007; Ignatova, Seregin, 2007; Werner et al., 2007; HedenAs, RosborG 2008; Ros et al., 2008, 2013; BOIKO, 2009, 2010, 2011, 2012; VANDERPOORTEN, HEDENAS 2009; KonstantinOva et al., 2009; KonstantinOva, Vilnet, 2009; Bell, HyvOnEn, 2010; HolyoAK, 2010; HutTunen, IGNATOV, 2010; KOPONEN, ISOVIITA, 2010; LOBACHEVs'KA, SOKHANCHAK, 2010; Ros, HerRnStAdT, 2010; SÖDERSTRÖM, DE RoO, HedD., 2010; VIRCHENKO, PARTYKA, 2010; CROSBY, BUCK, 2011; OlSSON et al., 2011; RUBASINGHE et al., 2011 a, b; VILNET et al. 2011, 2012; ANDRJEEVA, 2012; BARSUKOV, VIRCHENKO, 2012; ELLIS et al., 2012; KUSJARIN, 2012; RAGULINA, ISIKOV, 2012; VIRCHENKO, 2012; VÁÑA et al., 2012, 2013 a, b; FeldBerg et al., 2013; BorovicheV, NyPOrKo, 2014; CheCKLIST..., 2014; Č́́HAL, 2014; PLAŠEK et al., 2014 etc].

\section{Marchantiophyta}

Asterella lindenbergiana (Corda ex Nees) Lindb. ex Arnell.

Barbilophozia sudetica (Nees ex Huebener) L. Soderstr., De Roo et Hedd. (Lophozia sudetica (Nees ex Huebener) Grolle, L. alpestris (Schleich.) Evans.).

Clevea hyalina (Sommerf.) Lindb. (Athalamia hyálina (Sommerf.) S. Hatt.).

Clevea spathysii (Lindenb.) K.Müll. (Athalamia spathýsii (Lindenb.) S. Hatt.).

Conocephalum salebrosum Szweyk., Buczk. et Odrzyk.

Mannia androgyna (L.) A. Evans.

Endogemma caespitícia (Lindenb.) Konstant., A.Vilnet et A.V.Troitsky (Solenostoma caespiticium (Lindenb.) Steph, Jungermannia caespiticia Lindenb.

Isopàches bicrenatus (Schmidel ex Hohhm.) H. Buch (Lophozia bicrenata (Schmid. ex Hoffm.) Dumort.

Leomylia anomala (Hook.) J.J. Engel et Braggins (Mylia anomála (Hook.) Gray Gray). Liochlaéna lanceolata Nees (Jungermannia leiántha Grolle, J. lanceolata L., Solenostoma lanceolata Steph.).

Liochlaena subulata (A.Evans) Schljakov (Jungermannia subulata A. Evans). Lophoziopsis excisa (Dicks.) Konstant. et Vilnet (Lophozia excisa (Dicks.) Dumort.). Lophoziopsis longidens (Lindb.) Konstant. et Vilnet (Lophozia longidens (Lindb.) Macoun.).

Mesoptychia badensis (Gottsche ex Rabenhorst.) L. Soderstr. et Vana (Lophozia badensis (Gottsche) Schiffn, Leiocolea badensis (Gott.) Joerg.).

Mesoptychia bantriensis (Hook.) L. Soderstr. et Vana (Lophozia bantriensis (Hook.) Steph., L. hornschuchiana (Nees) Mac, Leiocolea bantriensis (Hook.) Joerg., L. mulleri (Nees) Joerg.).

Mesoptychia collaris (Nees.) L. Soderstr. et Vana (Lophozia collaris (Nees) Dumort., Jungermannia collaris Nees.

Mesoptychia heterocolpos (Thed. ex C. Hartm.) L. Soderstr. (Lophozia heterocolpos (Thed. ex C. Hartm.) M. Howe, Leiocolea heterocolpos (Thed.) Buch).

Metzgeria pubescens (Schrank) Raddi (Apometzgeria pubescens (Schrank) Kuwah.).

Metzgeria violacea (Ach. ex F.Weber et D. Mohr) Dumort. (Metzgeria fruticulosa (Dicks.) A. Evans, M. furcata var. fruticulosa Lindb.).

Neoorthocaulis attenuatus (Mart.) L. Soderstr., De Roo et Hedd (Barbilophozia attenuata (Mart.) Loeske, B. gracilis (Schleich.) K. Müll.).

Neoorthocaulis floerkei (F. Weber \& Mohr) L. Soderstr., De Roo et Hedd (Barbilophozia floerkei (F. Weber \& Mohr) Loeske).

Riccia beyrichiana Hampe ex Lehm.

Riccia crinita Hoffm.(R. trichocarpa M.Howe, R. canescens Steph.).Hoffm.

Riccia erinacea Schiffn.

Riccia rhenana Lorbeer var. violacea Boiko. 
Schistochilopsis incisa (Schrad.) Konstant. 1994 (Lophozia incisa (Schrad.) Dumort.).

Schljacovianthus quadrilobus (Lindb.) 2010 (Barbilophozia quadriloba (Lindb.) Loeske.)

Solenostoma confertissimum (Nees) Schljakov) 1980 (Jungermannia confertissima Nees, Solenostoma levieri (Steph.).

Solenostoma gracillimum (Sm.) R.M. Schust. (Plectocolea crenulata Evans, Jungermannia gracillima Sm., Solenostoma crenulatum (Sm.) Mitt.).

Solenostoma hyalinum (Lyell ex Hook.) Mitt. (Plectocolea hyalina (Lyell) Mitt. (Jungermannia hyalina Lyell).

Solenostoma obovatum (Nees) C. Massal. 1903 (Plectocolea obovata (Nees) Mitt., Jungermannia obovata Nees).

Solenostoma sphaerocarpum (Hook.) Steph. (Jungermannia sphaerocarpa Hook., Solenostoma sphaerocarpum (Hook.) Steph.).

Sphenolobus minutus (Schreb.) Berggr. (Anastrophyllum minútum (Schreb.) R. M. Schust.).

Syzygiella autumnalis (DC.) K. Feldberg, Vana, Hentschel et Heinrichs 2010 (Jamesoniella autumnális (DC.) Steph.).

Trilophozia quinquedentata (Huds.) Bakalin (Tritomaria quinquedentata (Huds.) H. Buch, Barbilophozia quinquedentata Loeske).

\section{Bryophyta}

Alleniella besseri (Lobarz.) S.Olsson, Enroth \& D.Quandt (Neckera besseri (Lob.) Jur., Homalia besseri Lobarz.).

Alleniella complanata (Hedw.) S.Olsson, Enroth \& D.Quandt (Neckera besseri (Lob.) Jur., Homalia besseri Lobarz.).

Aulacomnium arenopaludosum Boiko.

Bryum badium (Brid.) Schimp.

Campylidium calcareum (Crundw. \& Nyholm) Ochyra (Campylophyllum calcareum (Crundw. \& Nyholm) Hedenäs, Campylium calcareum Crundw. \& Nyholm)

Campylidium sommerfeltii (Myrin) Ochyra Campylophyllum sommerfeltii (Myrin) Hedenäs, Chrysohypnum sommerfeltii (Myrin) Roth).

Campylopus introflexus (Hedw.) Brid.

Dichelyma capillaceum (L. ex Dicks.) Myrin.

Dichodontium flavescens (Dicks.) Lindb.

Drepanocladus lycopodioides (Brid.) Warnst. (Pseudocalliergon lycopodioides (Brid.) Hedenäs.

Drepanocladus polycarpos (Blandow ex Voit) Warnst. (D. aduncus var. polycarpus (Bland.)Moenk.).

Drepanocladus trifarius (F.Weber \& D.Mohr) Broth. ex Paris (Pseudocalliergon trifarium (F.Weber \& Mohr) Loeske, Calliergon trifarium (F.Weber \& D.Mohr) Kindb.).

Ephemerum crassinervium subsp. sessile (Bruch) Holyoak (Ephemerum sessile (Bruch) H. Müll.).

Exsertotheca crispa (Hedw.) S.Olsson, Enroth \&D.Quandt (Neckera crispa Hedw.).

Grimmia fuscolutea Hook.

Hymenoloma compactum (Schleich. ex Schwagr.) Ochyra (Dicranoweisia compacta (Schleich. ex Schwaegr.) Schimp., D. crispula var. compacta (Schwägr.) Lindb.).

Hymenoloma crispulum (Hedw.) Ochyra (Dicranoweisia crispula (Hedw.) Milde).

Imbribryum alpinum (Huds. ex With.) N. Pedersen (Bryum alpinum Huds. ex With.)

Imbribryum mildeanum (Jur.) J.R. Spence (Bryum mildeanum Jur., B. alpinum var. mildeanum (Jur.) Podp.).

Lazarenkia kozlovii (Lazar.) Boiko (Pterygoneurum kozlóvii Lazar.). 
Lescuraea incurvata (Hedw.) E. Lawton (L. atrovirens (Bruch \& Schimp.) E. Lawto, Pseudoleskea incurvata (Hedw.) Loeske).

Lescuraea patens Lindb. (Pseudoleskea patens (Lindb.) Kindb.).

Lescuraea radicosa (Mitt.) Monk. (Pseudoleskea radicosa (Mitt.) Macoun \& Kindb.

Myrinia pulvinata (Wahlendb.) Schimp.

Nogopterium gracile (Hedw.) Crosby \& W.R.Buck. (Pterogonium gracile (Hedw.) Sm.).

Nyholmiella gymnostoma (Bruch ex Brid.) Holmen \& Warncke(Orthotrichum gymnostomum Bruch ex Brid.).

Nyholmiella obtusifolia (Brid.) Holmen \& Warncke (Orthotrichum obtusifolium Brid.).

Orthotrichum affine var. bohemicum Plašek \& Sawicki.

Philonotis capillaris Lindb. (Ph. arnellii Husn., Ph. fontana subsp. capillaris (Lindb.) Herib.).

Plagiothecium denticulatum (Hedw.) Schimp. var. undulátum R.Ruthe ex Geh. (Plagiothecium ruthei Limpr.).

Pohlia proligera (Kindb.) Lindb.

Polytrichum formosum Hedw. (Polytrichastrum formosum Hedw G. Sm.).

Polytrichum longisetum Sw. ex Brid. (Polytrichastrum longisetum (Sw. ex Brid.) G. Sm.).

Polytrichum pallidisetum Funck (Polytrichastrum pallidisetum (Funck) G. Sm.).

Polytrichum swartzii Hartm. (P. uliginosum (Wallr.) Schriebl, P. commune var. swartzii (Hartm.) Nyholm).

Pseudocampýlium radicále ( $\mathrm{P}$. Beauv Vanderp. \& Hedenäs. (Amblystegium radicale ( $P$. Beauv.) Schimp., A. saxatile Schimp., Campylium radicale (P.Beauv.) Grout) -

Pseudoamblystegium subtile (Hedw.) Vanderp. \& Hedenas (Amblystegium subtile (Hedw.) Schimp.).

Pseudocampylium radicale (P. Beauv.) Vanderp. \& Hedenas. (Amblystegium radicale (P. Beauv.) Schimp.).

Ptychostomum archangelicum (Bruch \& Schimp.) J.R. Spence. (Bryum archangelicum Bruch \& Schimp).

Ptychostomum boreale (F.Weber \& D.Mohr) Ochyra \& Bednarek-Ochyra (Bryum pallescens Schleich. ex Schwaegr., B. cirratum Hoppe \& Horhsch.).

Ptychostomum capillare (Hedw.) Holyoak \& N.Pedersen (Bryum capillare Hedw.).

Ptychostomum cernuum (Hedw.) Hornsch. (Bryum cernuum (Hedw.) Bruch \& Schimp.). Ptychostomum compactum Hornsch. (B. compactum (Hornsch.) Kindb.).

Ptychostomum creberrimum (Taylor) J.R. Spence \& H.P. Ramsay. (Bryum creberrimum Taylor).

Ptychostomum imbricatulum (Mull.Hal.) Holyoak \& N. Pedersen.(Bryum imbricatum (Schwaegr.) Bruch \& Schimp.).

Ptychostomum moravicum (Podp.) Ros \& Mazimpaka. (Bryum moravicum Podp.).

Ptychostomum pallens (Sw.) J.R.Spence (Bryum pallens Sw.).

Ptychostomum pseudotriquetrum (Hedw.) J.R. Spence \& H.P. Ramsay (Bryum pseudotriquetrum (Hedw.) P. Gaertn. et al.).

Ptychostomum pseudotriquetrum (Hedw.) J.R. Spence \& H.P. Ramsay var. bímum (Schreb.) Holyoak \& N. Pedersen (Bryum bimum (Brid.) Turn.).

Ptychostomum rubens (Mitt.) Holyoak \& N. Pedersen (Bryum rubens Mitt.).

Ptychostomum torquescens (Bruch \& Schimp.) Ros \& Mazimpaka. (Bryum torquescens Bruch \& Schimp.).

Ptychostomum zieri (Hedw.) Holyoak \& N. Pedersen (Plagiobryum zieri (Hedw.) Lindb.).

Rhynchostegiella teneriffae (Mont.) Dirkse \& Bouman. 
Rhynchostegium riparioides (Hedw.) Cardot (Platyhypnidium riparióides (Hedw.) Dix. P. rusciforme Fleisch., Eurhynchium rusciforme (Hedw.) Dix.).

Sarmentypnum exannulatum (Schimp.) Hedenas. (Warnstorfia exannulata (Schimp.) Loeske).

Sarmentypnum sarmentosum (Wahlenb.) Tuom. \& T.J. Kop. (Warnstorfia sarmentosa (Wahlenb.) Hedenäs).

Schistidium crassipilium Blom.

Schistidium elengatulum Blom.

Schistidium dupretii (Ther.) W.A.Weber.

Schistidium lancifolium (Kind.) Blom.

Schistidium pulchrum Blom.

Schistidium robustum (Nees \& Hornsch.) Blom.

Schistidium trichodon (Brid.) Poelt var. nutans Blom.

Sciuro-hypnum curtum (Lindb.) Ignatov (Brachythecium curtum (Lindb.).

Serpoleskea confervoides (Brid.) Schimp. (Platydictya confervoides (Brid.) H.A.Crum).

Syntrichia laevipila Brid.

Syntrichia ruralis (Hedw.) F. Weber \& Mohr var. polysporogonica Boiko.

Tortella squarrosa (Brid.) Limpr.

Tortula acaulon (With.) R.H. Zander (Phascum cuspidatum Hedw.).

Tortula caucasica Broth. (Tortula modica Zander).

Tortula lindbergii Broth. (Tortula lanceola R. H. Zander).

Tortula protobryoides R.H. Zander (Pottia bryoides (Dicks.) Mitt.).

\section{Doubtful taxa}

Grimmia fuscolutea Hook. species has many synonyms, the most famous of them are G. apiculata Hornsch., G. fastigiata Cardot, G. flexicauli C. Müll. and others. Species was presented for Ukrainian Carpathians valley of Dvorysh stream near Vyzhenka village Vyzhnytskyi district of Chernivtsi region where it grew up in silicate rocks [STEFURAC, 1936]. This habitat of the species has been shown in a number of papers [LAZARENKO, 1955; ZEROV, PARTYKA, 1975; BACHURYNA, MELNYCHUK, 1988]. However, V.M. Melnychuk [MELNYCHUK, 1970; BACHURYNA, MELNYCHUK, 1988] expressed some doubt about the findings. In our view the species should be included into checklist, but with awareness, because sufficient objections about inclusion are absent for now. Another indication of the growth of this species in Ukrainian Carpathians - Transcarpathian region, Mount Petros was wrong [BACHURYNA, MELNYCHUK, 1988].

Lunularia cruciata (L.) Dumort. Species was presented by D.K. Zerov [1964] for Kyiv. It grows on the ground near greenhouses of O.V. Fomin Botanical Garden with heatloving plants that are planted in the summer ground. Naturally species grows in the Mediterranean region, in America and Australia it is considered wild, in Western and Central Europe it is found near the greenhouses and baths. You can consider it as an alien species, but its presence in Ukraine shouldn not be ignored [VÁŇA, VIRCHENKO, 1993]. Especially in the last decade a lot of plant material for cultivation in botanical gardens and private yard has come from Europe to Ukraine. Therefore, an indication of the species growth in Ukraine should be kept, but it is necessary to clarify the detailed studies of relevant habitats.

\section{Mosses taxa that were incorrectly or insufficiently substantiated for the territories of Ukraine in the European Checklist of bryophytes (Checklist ..., 2014)}

\section{Marchantiophyta}

Asterella saccata (Wahlenb.) A. Evans. There was only the possibility of finding the species in Ukraine indicated, as it was noted in neighboring Eastern European countries [ZEROV, 1964]. 
Bazzania flaccida (Dumort.) Grolle (= Bazzania denudata auct.) Species wasn't marked in Ukraine. D.K. Zerov [1964] only pointed out that the species was found in the neighborhood - the Tatra Mountains.

Bucegia romanica Radian. The lack of this species in Ukraine was proved earlier [VÁŇA, VIRCHENKO, 1993].

Cepalozia lacinulata (JBJack ex Gottsche \& Rabenh.) Spruce. D.K. Zerov [1964] doubted the accuracy of sample determination of $C$. lacinulata of the Ukrainian Carpathians as in sterile condition it was difficult to determine. Later I. Ván̆a indicated the species as Cephaloziella divaricata [VÁŇA, VIRCHENKO, 1993].

Cepalozia macrostachia Kaal. The possibility of finding the species in Ukraine was noted [ZEROV, 1964].

Cephaloziella divaricata var. scabra (M. Howe) Haynes. Species is given in the works of D.K. Zerov [1964] and I. Váňa, V.M. Virchenko [1993] according to the literature data [KRUPA, 1885; REJMENT-GROCHOWSKA, 1958] without giving a specific locations for var. scabra.

Jungermannia exsertifolia Steph. [subsp. cordifolia (Dumort.) Ván̆a). The sample, as Haplozia cordifolia shown by D.K. Zerov [1964] according to the Boros' work [BOROS, 1951]. Sample was redefined as Jungermannia pumila f. rivularis [VÁŇA, VIRCHENKO, 1993].

Lophocolea coadunata Steph. (= L. bidentata).

Lophozia silvicola (= L. ventricosa, Lophozia ventricosa var. silvicola $(\mathrm{H}$. Buch) E.W. Jones.

Mesoptychia gilmanii H. Buch (synonym Lophozia gilmanii). Species was indicated for Ukraine by mistake [DÜLL, 1983].

Nardia compressa (Hook.) Gray. After redefining sample was named as Nardia scalaris [VÁŇA, VIRCHENKO, 1993].

Nardia insecta Lindb. After redefining the sample was defined as Nardia geoscyphus [VÁŇA, VIRCHENKO, 1993].

Obtusifolium obtusum (Lindb.) S.Arnell - (= Lophozia obtusa (Lindb.) A. Evans, Leiocolea obtusa (Lindb.) Buch). Species was specified for Ukraine by mistake [DÜLL, 1983].

Peltolepis quadrata [BOROS, VAIDA, 1968]. After redefining the sample was defined as Mannia pilosa [VÁŇA, VIRCHENKO, 1993].

Riccia glauca L. var. ciliaris Warnst. Species is provided in the work of D.K. Zerov [1964] as Riccia glauca var. subinermis (Lindb.) Warnst. (Riccia subinermis Lindb.) Without giving a specific locations. It is necessary to study all available materials for certain judgments.

Riccia perennis Steph. Species was defined for Ukraine by mistake [DÜLL, 1983].

Scapania paludosa (Mül.Frib.) Mül.Frib. D.K. Zerov [1964] indicates the type of proximity to $S$. undulata, but does not specify the location, but suggests that the species occurs in the subalpine zone. S. paludosa indicated for the Ukrainian Carpathians was [SLOBODYAN, 1950] redefined as S. undulata [VÁŇA, VIRCHENKO, 1993].

Scapania scandica (Arnell \& H.Buch) Macvicar. It was only indicated the closeness of this species to $S$. curta, but the possibility of finding the species in Ukraine was not mentioned [ZEROV, 1964].

Schistochilopsis incisa var. inermis (Müll. Frib.) Konstant. (= Lophozia incisa (Schrad.) Dumort. var. inermis K.Müll. In Rabenh.). Species was indicated only in general terms, location was not listed in Ukraine [ZEROV, 1964, VÁŇA, VIRCHENKO, 1993].

Schistochilopsis opacifolia (Culm. ex Meyl.) Konstant. (S. incisa var. opacifolia). There is no information on finding this arch-alpine species in Ukraine.

Schljakovia kunceana (Huebener) Konstant. \& Vilnet (= Barbilophozia kunzeana (Huebener) Müll. Frib.). There is no information on finding this arch-alpine species in Ukraine. 
Solenostoma pusillum (C.E.O. Jensen) Steph. Only the growth of this species in Transylvania (Romania) was mentioned and the possibility of finding the species in Ukraine was indicated [ZEROV, 1964].

Southbya tophacea (Spruce) Spruce (Jungermannia tophacea). Species was given to Ukraine by mistake [DÜLL, 1983].

Sphenolobus saxicola (Schrad.) Steph. (= Anastrophyllum saxicola (Schrad.) Schust.). Species was indicated for the Ukrainian Carpathians [BOROS, VAJDA, 1968]. The material was redefined as Anastrophyllum michauxii (F.Weber) H. Buch [VÁŇA, VIRCHENKO, 1993].

\section{Bryophyta} Ukraine.

Bryum gemmilucens R.Wilczek \& Demaret. There is no information on this species in

Plagiothecium denticulatum var. obtusifolium (Turner) Moore. There is no information on finding this species in Ukraine, it is known from Alaska, China, and Japan.

Pohlia elongata var. greenii (Brid.) Shaw (= Pohlia elongata var. minor Hartm., Pohlia minor Schleich ex Schwaegr). There is no information on finding this species in Ukraine.

Ptychostomum compactum var. rutheanum (Warnst.) Holyoak \& N.Pedersen. There is no information on finding this species in Ukraine.

\section{Excluded species from brioflora of Ukraine}

Pohlia andrevsii J. Shaw. Species was presented for Ukraine by M.S. Ihnatov and O.M. Afonina in Checklist of mosses of the former USSR in 1992. However in later works of these and other authors this species had not been indicated any more. Thus this indication could be considered as wrong. V.M.Virchenko [2010] offers to exclude the species from the bryophyte flora of Ukraine. We [BOIKO, 2012], supported this proposal, but noted that the probability of finding of this species in the country was very high.

Schistidium confertum (Funck) Bruch \& Schimp. This type is specified for many lowland and mountain habitats of phyto-geographical regions of Ukraine [BACHURYNA, MelnyChuK, 1988; Boiko, 2008]. H. Blom [BLOM, 1996] describes the species as montaneous, with nearly circumpolar distribution. Distributed in the mountains of southern Europe. Noted in the mountainous regions from eastern Ardennes to Poland. There are localities in northern Europe - Wales, Scotland, Iceland. Also in the mountains of the Caucasus, Turkey, Armenia, in the Himalayas and Tibet, in the mountains of North America, Alaska. All materials are known in Ukraine should be redefined according to the current understanding of the species and decide on the presence or absence of this species in Ukraine.

Schistidium strictum (Turn.) Loeske - an oceanic species, found in Norway, the Faroe Islands, the western part of Iceland, the western part of the United Kingdom (Wales, England, Scotland), Spain - Pyrenees $2000 \mathrm{~m}$ above sea level, Madeira - $1800 \mathrm{~m}$. In America hyperoceanic species of the Pacific coast: Canada - Vancouver, USA - Alaska, Aleutian Islands [BLOM, 1996]. In Ukraine probably it was never found. All specimens of this species in the herbarium of Ukraine must be determined again.

Sciuro-hypnum oedipódium (Mitt.) Ignatov \& Huttunen. In Ukraine Bryofloras, like in other countries bryofloras, Brachytecium curtum (Lindb.) J. Lange for a long time this species was not considered as an independent one and accepted as a form of B. starkei (Brid.) BSG, namely - B. starkei var. explanatum (Brid.) Mönk. [LAZARENKO, 1955; BACHURINA, MELNYCHUK, 2003]. O.I.Vysotska [1984] estimated that B. curtum and B. starkei are different chromosomal races, and there is a clear morphological differences between them. In addition, both species have a different distribution. V.M.Virchenko [2000] considers B. starkei (Brid.) BSG var. explanatum auct. and B. curtum (Lindb.) J. Lange et C. Jens. interchangeably to Brachytecium oedipodium (Mitt.) A. Jaeg. M.F. Boiko [2008, 2009] considered the species 
B. curtum (Lindb.) Limpr. and B. oedipodum (Mitt.) Jager. and B. starkei var. curtum (Lindb.) Warnst. and B. starkei var. explanatum (Brid.) Mönk. interchangeably to Sciuro-hypnum oedipódium (Mitt.) Ignatov \& Huttunen. M.S. Ignatov and I.A. Milutina [IgNATOV, MiLUTINA, 2007] based on molecular sequence data of ITS confirmed that Sciuro-hypnum oedipodium and Sciuro-hypnum curtum are separate species. That is, Sciuro-hypnum curtum, Sciuro-hypnum starkei and Sciuro-hypnum oedipodium at this stage are recognized as independent species. Since the distribution of non-distinguished species in Ukraine is not cleared (need to work out all the herbarium materials), today $S$. curtum and $S$. starkei can be only indicated for Ukrainian bryoflora. $S$. oedipodium is apparently absent in Ukraine, it is common in western North America, absent in its east, found in the Caucasus and Chukotka [IGNATOV, MilutinA, 2007]. In The new European checklist of bryophytes [CHECKLIST ..., 2014] S. oedipodium is not specified for Ukraine.

\section{Synonyms}

\section{Anthocerotophyta, Marchantiophyta}

Anastrophyllum hellerianum $=$ Crossocalyx hellerianum

Anastrophyllum minútum $=$ Sphenolobus minutes

Aneura major $=$ Riccardia chamaedryfolia

Aneura incurvata $=$ Riccardia incurvata

Aneura latifrons $=$ Riccardia latifrons

Aneura multifida $=$ Riccardia multifida

Aneura palmata $=$ Riccardia palmata

Athalamia hyalina $=$ Clevea hyalina

Athalamia spathysii $=$ Clevea spathysii

Anthoceros laevis $=$ Phaeoceros laevis

Anthoceros punctatus var. cavernosus $=$ Anthoceros agrestis

Apometzgeria pubescens $=$ Metzgeria pubescens

Barbilophozia attenuata $=$ Neoorthocaulis attenuates

Barbilophozia floerke $=$ Neoorthocaulis floerkei

Barbilophozia gracilis $=$ Neoorthocaulis attenuatus

Barbilophozia quadriloba $=$ Schljacovianthus quadriilobus

Barbilophozia quinquedentata $=$ Trilophozia quinquedentata

Calypogeia meylanii $=$ Calypogeia integristipula

Calypogeia neesiana var. meylanii = Calypogeia integristipula

Calypogeia trichomanis $=$ Calypogeia azurea

Cephalozia bicuspidata subsp. lammersiana $=$ Cephalozia lammersiana

Cephalozia fluitans $=$ Cladopodiella fluitans

Cephalozia media = Cephalozia lunulifolia

Cephalozia reclusa $=$ Cephalozia catenulata

Cephaloziella starkei $=$ Cephaloziella divaricata

Cephaloziella subdentata $=$ Cephaloziella spinigera

Chiloscyphus coadunatus $=$ Lophocolea bidentata

Chiloscyphus pallescens var. fragilis $=$ Chiloscyphus fragilis

Chiloscyphus polyanthus var. pallescens $=$ Chiloscyphus pallescens

Chiloscyphus polyanthus var. rivularis $=$ Chiloscyphus rivularis

Frullania nervosa $=$ Frullania tamarisci

Fuscocephaloziopsis albescens $=$ Pleurocladula albescens

Gymnomitrion apiculatum = Marsupella apiculata

Haplozia atrovirens $=$ Jungermannia atrovirens

Haplozia pumila = Jungermannia pumila

Jamesoniella autumnalis $=$ Syzygiella autumnalis 
Jungermannia caespiticia $=$ Endogemma caespiticia

Jungermannia collaris $=$ Mesoptychia collaris

Jungermannia confertíssima $=$ Solenostoma confertissimum

Jungermannia gracillima $=$ Solenostoma gracillimum

Jungermannia hyalina $=$ Solenostoma hyalinum

Jungermannia juratzkana = Anthelia juratzkana

Jungermannia leiantha $=$ Liochlena lanceolata

Jungermannia lanceolata $=$ Liochlaena lanceolata

Jungermannia obovata $=$ Solenostoma obovatum

Jungermannia sphaerocárpa $=$ Solenostoma sphaerocarpum

Jungermannia subuláta $=$ Liochlena subulata

Leiocolea badensis = Mesoptychia badensis

Leiocolea bantriensis $=$ Mesoptychia bantriensis

Leiocolea heterocolpos $=$ Mesoptychia heterocolpos

Leiocolea mulleri $=$ Mesoptychia bantriensis

Mylia anomala $=$ Leomylia anomala

Lophocolea coadunate $=$ Lophocolea bidentata

Lophozia alpestris $=$ Barbilophozia sudetica

Lophozia badénsis $=$ Mesoptychia badensis

Lophozia bantriensis = Mesoptychia bantriensis

Lophozia bicrenata $=$ Isopaches bicrenatus

Lophozia collaris $=$ Mesoptychia collaris

Lophozia confertifolia = Lophozia wenzelii

Lophozia excisa = Lophoziopsis excisa

Lophozia heterocolpos $=$ Mesoptychia heterocolpos

Lophozia hornschuchiana = Mesoptychia bantriensis

Lophozia incisa $=$ Schistochilopsis incisa

Lophozia longidens $=$ Lophoziopsis longidens

Lophozia longiflora $=$ Lophozia guttulata

Lophozia porphyroleuca = Lophozia guttulata

Lophozia sudetica $=$ Barbilophozia sudetica

Lophozia ventricosa var. confertifolia $=$ Lophozia wenzelii

Mannia rupestris $=$ Mannia triandra

Marchantia polymorpha subsp. montivagans = Marchantia alpestris

Marsupella adusta = Gymnomitrion adustum

Marsupella badensis = Marsupella funckii

Metzgeria fruticulosa $=$ Metzgeria violacea

Metzgeria furcata var. fruticulosa $=$ Metzgeria violacea

Metzgeria hamata $=$ Metzgeria leptoneura

Mylia anomala $=$ Leomylia anomala

Oxymitra paleacea $=$ Oxymítra incrassata

Pellia fabroniana $=$ Pellia endiviifolia

Phaeoceros laevis subsp. carolinianus $=$ Phaeoceros carolinianus

P. asplenioides subsp. porelloides $=$ Plagiochila porelloides

Plagiochila major = Plagiochila asplenioides

Plectocolea crenulata $=$ Solenostoma gracíllimum

Plectocolea hyalina $=$ Solenostoma hyalinum

Plectocolea obovata $=$ Solenostoma obovatum

Porella laevigata $=$ Porella arboris-vitae

Riccardia pinguis $=$ Aneura pinguis

Riccardia sinuata $=$ Riccardia chamaedryfolia 
Riccia canescens $=$ Riccia crinita

Riccia gougetiana var.armatissima $=$ Riccia erinacea

Riccia gougetiana var erinacea $=$ Riccia erinacea

Riccia trichocarpa $=$ Riccia crinita

Riccia pseudopapillosa = Riccia papillosa

Scapania nemorosa = Scapania nemorea

Solenostoma caespiticium $=$ Endogemma caespiticia

Solenostoma crenulatum $=$ Solenostoma gracillimum

Plectocolea hyalina $=$ Solenostoma hyalinum

Solenostoma lanceolata $=$ Liochlaena lanceolata

Solenostoma levieri $=$ Solenostoma confertíssimum

Solenostoma pumila = Jungermannia pumila

Telaranea setacea $=$ Kurzia pauciflora

Tritomaria quinquedentáta $=$ Trilophozia quinquedentata

Acaulon rubrum $=$ Acaulon muticum

Amblystegiella confervoides $=$ Serpoleskea confervoides

Amblystegium confervoides $=$ Serpoleskea confervoides

Amblystegiella spruce $i=$ Platydictya jungermannioides

Amblystegiella subtilis $=$ Pseudoamblystegium subtile

Amblystegium compactum = Conardia compacta

Amblystegium humile $=$ Hygroamblystegium humile

Amblystegium jungermannioides $=$ Platydictya jungermannioides

Amblystegium kochii $=$ Hygroamblystegium humile

Amblystegium radicale $=$ Pseudocampylium radicale

Amblystegium riparium $=$ Leptodictyum riparium

Amblystegium saxatile $=$ Pseudocampylium radicale

Amblystegium subtile $=$ Pseudoamblystegium subtile

Amblystegium tenax $=$ Hygroamblystegium tenax

Amblystegium varium $=$ Hygroamblystegium varium

Anoectangium hornschuchianum $=$ Molendoa hornschuchiána

Anoectangium sendtnerianum $=$ Molendoa sendtneriana

Anomodon apiculatus $=$ Anomodon rugelii

Astomum crispum $=$ Weissia longifolia

Astomum crispum var. levieri $=$ Weissia levieri

Astomum crispum var. phylibertii $=$ Weissia levieri

Atrichum undulatum var. haussknechtii = Atrichum flavisetum

Atrichum undulatum var. minor $=$ Atrichum crispum

Barbula acuta $=$ Didymodon acutus

Barbula cordata $=$ Didimodon cordatus

Barbula cylindrica $=$ Didymodon insulanus

Barbula fallax $=$ Didymodon fallax

Barbula hornschuchiana $=$ Pseudocrossidium hornschuchianum

Barbula lurida $=$ Didymodon luridus

Barbula reflexa $=$ Didymodon ferrugineus

Barbula revoluta $=$ Pseudocrossidium revolutum

Barbula rigidula $=$ Didymodon rigidulus

Barbula sinuosa $=$ Didymodon sinuosus

Barbula spadicea $=$ Didymodon spadiceus

Barbula tophacea $=$ Didymodon tophaceus 
Barbula vinealis $=$ Didymodon vinealis

Bartramia oederi $=$ Plagiopus oederianus

Brachythecium curtum $=$ Sciuro-hypnum curtum

Brachythecium plumosum $=$ Sciuro-hypnum plumosum

Brachythecium populeum $=$ Sciuro-hypnum populeum

Brachythecium reflexum $=$ Sciuro-hypnum reflexum

Brachythecium trachypodium = Brachytheciastrum trachypodium

Brachythecium starke $i=$ Sciuro-hypnum starkei

Brachythecium velutinum $=$ Brachytheciastrum velutinum

Breidleria arcuata $=$ Calliergonella lindbergii

Bryoerythrophyllum recurvirostrum var. dentatum $=$ Bryoerythrophyllum alpigenum

Bryum algovicum $=$ Ptychostomum compactum

Bryum alpinum $=$ Imbribryum alpinum

Bryum alpinum subsp. gemmiparum = Bryum gemmiparum

Bryum alpinum var. mildeanum $=$ Imbribryum mildeanum

Bryum angustirete $=$ Ptychostomum compactum

Bryum amblyodon $=$ Ptychostomum imbricatulum

Bryum argenteum subsp. veronense $=$ Bryum veronense

Bryum archangélicum $=$ Ptychostomum archangelicum

Bryum bimum $=$ Ptychostomum pseudotriquetrum var. bimum

Bryum capillare $=$ Ptychostomum capillare

Bryum capillare var. elegans $=$ Bryum elegans

Bryum capillare var. flaccidum $=$ Ptychostomum moravicum

Bryum capillare var. torquescens $=$ Ptychostomum torquescens

Bryum cernuum $=$ Ptychostomum cernuum

Bryum cirratum $=$ Ptychostomum boreale

Bryum compactum $=$ Ptychostomum compactum

Bryum creberrimum $=$ Ptychostomum creberrimum

Bryum flaccidum $=$ Ptychostomum pallens

Bryum intermedium var. pallens $=$ Ptychostomum pallens

Bryum imbricatum $=$ Ptychostomum imbricatulum

Bryum julaceum $=$ Anomobryum julaceum

Bryum lacustre $=$ Bryum knowltonii

Bryum mildeanum $=$ Imbribryum mildeanum

Bryum moravicum $=$ Ptychostomum moravicum

Bryum pallescens $=$ Ptychostomum boreale

Bryum pallens $=$ Ptychostomum pallens

Bryum pendulum = Ptychostomum compactum

Bryum pendulum subsp. archangelicum = Ptychostomum archangelicum

Bryum pseudotriquetrum = Ptychostomum pseudotriquetrum

Bryum rubens $=$ Ptychostomum rubens

Bryum subelegans $=$ Ptychostomum pallens

Bryum torquescens $=$ Ptychostomum torquescens

Bryum uliginósum = Ptychostomum cernuum

Calliergon sarmentosum $=$ Sarmentypnum sarmentosum

Calliergon stramineum $=$ Straminergon stramineum

Calliergon trifarium $=$ Drepanocladus trifarius

Campilopus fragilis var. pyriformis $=$ Campilopus pyriformis

Camptothecium aureum $=$ Homalothecium aureum

Camptothecium lutescens $=$ Homalothecium lutescens

Camptothecium nitens $=$ Tomentypnum nitens 
Campyliadelphus stellatus $=$ Campylium stellatum

Campylium calcareum $=$ Campylidium calcareum

Campylophyllum calcareum $=$ Campylidium calcareum

Campylium chrysophyllum = Campyliadelphus chrysophyllus

Campylium elodes $=$ Campyliadelphus elodes

Campylium halleri $=$ Campylophyllum halleri

Campylium polygamum $=$ Drepanocladus polygamus

Campylium radicale $=$ Pseudocampylium radicale

Campylophyllum calcareum $=$ Campylidium calcareum

Campylophyllum sommerfeltii $=$ Campylium sommerfeltii

Campylopus fragilis var. pyriformis $=$ Campilopus pyriformis

Campylopus saxicola $=$ Campylostelium saxicola

Campylopus schwarzii = Campilopus gracilis

Chrysohypnum helodes $=$ Campyliadelphus elodes

Chrysohypnum sommerfeltii $=$ Campylium sommerfeltii

Cinclidotus nigricans $=$ Cinclidotus riparius

Cirriphyllum cirrosum $=$ Brachythecium cirrosum

Cirriphyllum reichenbachianum $=$ Sciuro-hypnum flotowianum

Cirriphyllum tommasinii $=$ Brachythecium tommasinii

Cirriphyllum vaucheri $=$ Brachythecium tommasinii

Cratoneuron commutatum $=$ Palustriella commutata

Cratoneuron decipiens $=$ Palustriella decipiens

Cratoneuron falcatum $=$ Palustriella falcata

Cratoneuron filicinum var. curvicaule $=$ Cratoneuron curvicaule

Cynodontium flexicaule $=$ Ditrichum flexicaule

Cynodontium inclinatum $=$ Distichium inclinatum

Cynodontium schistii $=$ Cnestrum schistii

Cynodontium virens $=$ Oncophorus virens

Cynodontium wahlenbergii $=$ Oncophorus wahlenbergii

Desmatodon cernuus $=$ Tortula cernua

Desmatodon heimii $=$ Hennediella heimii

Desmatodon latifolius $=$ Tortula hoppeana

Desmatodon leucostomus $=$ Tortula leucostoma

Desmatodon randii $=$ Tortula randii

Desmatodon ucrainicus $=$ Tortula ucrainica

Dicranella curvata $=$ Dicranella subulata

Dicranella palustris $=$ Dichodontium palustre

Dicranodontium longirostre $=$ Dicranodontium denudatum

Dicranoweisia compacta $=$ Hymenoloma compactum

Dicranoweisia crispula $=$ Hymenoloma cripulum

Dicranoweisia crispula var. compacta $=$ Hymenoloma compactum

Dicranum affine $=$ Dicranum undulatum

Dicranum bergeri $=$ Dicranum undulatum

Dicranum elongatum subsp. groenlandicum $=$ Dicranum groenlandicum

Dicranum enerve $=$ Paraleucobryum enerve

Dicranum fulvellum $=$ Arctoa fulvella

Dicranum fulvum var. viride $=$ Dicranum viride

Dicranum fuscescens var. flexicaule = Dicranum flexicaule

Dicranum longifolium $=$ Paraleucobryum longifolium

Dicranum muehlenbeckii var. spadiceum $=$ Dicranum spadiceum

Dicranum rugosum $=$ Dicranum polysetum 
Dicranum sendtneri $=$ Dicranum elongatum

Didymodon alpigenum = Bryoerythrophyllum alpigenum

Didymodon flexicaule $=$ Ditrichum flexicaule

Ditrichum cylindricum $=$ Trichodon cylíndricus

Ditrichum heteromallum var. zonatum = Ditrichum zonatum

Ditrichum tortile $=$ Ditrichum pusillum

Dolichotheca seligerii $=$ Herzogiella seligeri

Dolichotheca striatela $=$ Herzogiella striatella

Drepanocladus aduncus var. polycarpus $=$ Drepanocladus polycarpos

Drepanocladus cossonii $=$ Scorpidium cossonii

Drepanocladus exannulatus $=$ Sarmentypnum exannulatum

Drepanocladus fluitans $=$ Warnstorfia fluitans

Drepanocladus fluitans $f$. pseudostraminea $=$ Warnstorfia pseudostraminea

Drepanocladus intermedius $=$ Scorpidium cossonii

Drepanocladus uncinatus $=$ Sanionia uncinata

Drepanocladus revolvens $=$ Scorpidium revolvens

Drepanocladus vernicosus $=$ Hamatocaulis vernicosus

Dryptodon incurvus $=$ Grimmia elatior

Encalypta contorta $=$ Encalypta streptocarpa

Encalypta rhaptocarpa var. spathulata $=$ Encalypta spathulata

Encalypta vulgaris var. mutica $=$ Encalypta mutica

Ephemerum sessile $=$ Ephemerum crassinervium subsp. sessile

Erythrophyllum recurviróstrum $=$ Bryoerythrophyllum recurvirostrum

Eurhynchium circinatum $=$ Scorpiurium circinatum

Eurhynchium crassinervium $=$ Cirriphyllum crassinervium

Eurhynchium flotowianum = Sciuro-hypnum flotowianum

Eurhynchium hians $=$ Oxyrrhynchium hians

Eurhynchium meridionale $=$ Plasteurhynchium meridionale

Eurhynchium praelongum $=$ Kindbergia praelonga

Eurhynchium pulchellum = Eurhynchiastrum pulchellum

Eurhynchium schleicheri $=$ Oxirrhinchium schleicheri

Eurhynchium speciosum Oxyrrhynchium speciosum

Eurhynchium striatulum $=$ Plasteurhynchium striatulum

Eurhynchium striatulum var. meridionale $=$ Plasteurhynchium meridionale

Eurhynchium zetterstedtii $=$ Eurhynchium angustirete

Fissidens bryoides var. gymnandrus $=$ Fissidens gymnandrus

Fissidens cristatus $=$ Fissidens dubius

Fissidens decipiens $=$ Fissidens dubius

Fissidens exiguus $=$ Fissidens bryoides

Fissidens incurvus $=$ Fissidens bryoides

Fissidens mildeanus $=$ Fissidens crassipes

Fissidens minutulus $=$ Fissidens crispus

Fissidens obtusifolius $=$ Fissidens arnoldii

Fissidens viridulus var. pusillus $=$ Fissidens pusillus

Funaria dentata $=$ Entosthodon muhlenbergii

Funaria fascicularis $=$ Entosthodon fascicularis

Funaria hungarica $=$ Entosthodon hungaricus

Funaria muhlenbergii $=$ Entosthodon muhlenbergii

Grimmia acicularis $=$ Racomitrium aciculare

Grimmia agassizii = Schistidium agassizii

Grimmia campestris $=$ Grimmia laevigata 
Grimmia commutata $=$ Grimmia longirostris

Grimmia flaccida $=$ Schistidium flaccidum

Grimmia meridionalis $=$ Grimmia trichophylla

Grimmia ovalis $=$ Grimmia longirostris

Grimmia ovata $=$ Grimmia longirostris

Grimmia patens $=$ Grimmia ramondii

Grimmia tergestinoides $=$ Grimmia tergestina

Gymnostomum recurvirostrum = Hymenostylium recurvirostrum

Gymnostomum trichodes $=$ Brachydontium trichodes

Heterophyllium haldanianum $=$ Callicladium haldanianum

Heterophyllium nemorosum $=$ Heterophyllium affíne

Holmgrenia intricata $=$ Orthothecium intricatum

Homalia besseri $=$ Alleniella besseri

Homalia complanata $=$ Alleniella complanata

Homalothecium geheebii $=$ Brachythecium geheebii

Hylocomium brevirostre $=$ Loeskeobryum brevirostre

Hymenostomum crispatum $=$ Weissia controvéesa var. crispata

Hypnum cupressiforme var. ericetorum = Hypnum jutlandicum

Hypnum cupressiforme var. mammillatum $=$ Hypnum andoi

Hypnum cupressiforme var. vaucheri $=$ Hypnum vaucheri

Hypnum fastigiatum = Hypnum recurvatum

Hypnum lindbergii $=$ Calliergonella lindbergii

Hypnum pratense $=$ Breidleria pratensis

Hypnum reptile $=$ Hypnum pallescens

Isopterygium elegans $=$ Pseudotaxiphyllum elegans

Isopterygium muellerianum = Isopterygiopsis muelleriana

Isopterygium pulchellum = Isopterygiopsis pulchella

Isothecium myurum = Isothecium alopecuroides

Isothecium myosuroides var. rivulare $=$ Isothecium holtii

Isothecium viviparum $=$ Isothecium alopecuroides

Leskea intricata $=$ Orthothecium intricatum

Leskea mutabilis = Lescuraea mutabilis

Leskeella nervosa $=$ Pseudoleskeella nervosa

Leptodictyum humile = Hygroamblystegium humile

Leptodictyum kochii $=$ Hygroamblystegium humile

Lescuraea atrovirens $=$ Lescuraea incurvata

Lescuraea mutabilis var. saxicola $=$ Lescuraea saxicola

Leucobryum minus = Leucobryum juniperiodeum

Metaneckera menziesii $=$ Neckera menziesii

Mildeella bryoides $=$ Tortula protobryoides

Mniobryum albicans $=$ Pohlia wahlenbergii

Mniobryum carneum $=$ Pohlia melanodon

Mniobryum ludwigii = Pohlia ludwigii

Mniobryum pulchellum $=$ Pohlia lescuriana

Mniobryum wahlenbergii $=$ Pohlia wahlenbergii

Mnium affine var. elatum = Plagiomnium elatum

Mnium cinclidioides $=$ Pseudobryum cinclidiiides

Mnium longirostre $=$ Plagiomnium rostratum

Mnium medium $=$ Plagiomnium medium

Mnium punctatum $=$ Rhizomnium punctatum

Mnium punctatum var. elatum $=$ Rhizomnium magnifolium 
Mnium rostratum $=$ Plagiomnium rostratum

Mnium rugicum $=$ Plagiomnium ellipticum

Mnium seligeri $=$ Plagiomnium elatum

Mnium undulatum = Plagiomnium undulatum

Neckera besseri $=$ Alleniella besseri

Neckera crispa $=$ Exsertotheca crispa

Neckera complanata $=$ Alleniella complanata

Neckera fontinaloides $=$ Neckera pumila

Neckeradelphus menziesii $=$ Neckera menziesii

Octodiceras fontanum $=$ Fissidens fontanus

Oncophorus gracilescens $=$ Cynodontium gracilescens

Oncophorus polycarpus = Cynodontium polycarpon

Oncophorus strumifer $=$ Cynodontium strumiferum

Orthodicranum flagellare $=$ Dicranum flagellare

Orthodicranum fulvum = Dicranum fulvum

Orthodicranum montanum $=$ Dicranum montanum

Orthodicranum tauricum $=$ Dicranum tauricum

Orthotrichum fallax $=$ Orthotrichum pumilum

Orthotrichum fastigiatum $=$ Orthotrichum affine

Orthotrichum gymnostomum $=$ Nyholmiella gymnostoma

Orthotrichum leucomitrium $=$ Orthotrichum scanicum

Orthotrichum limprichtii = Orthotrichum cupulatum

Orthotrichum nudum= Orthotrichum cupulatum

Orthotrichum obtusifolium $=$ Nyholmiella obtusifolia

Orthotrichum pumilumDicks. $=$ Orthotrichum schimperi

Orthotrichum rogeri var. defluens $=$ Orthotrichum stramineum

Orthotrichum stramineum var. patens $=$ Orthotrichum patens

Paraleucobryum longifolium var. sauteri $=$ Paraleucobryum sauteri

Phascum acaulon $=$ Tortula acaulon

Phascum curvicollum $=$ Microbryum curvicollum

Phascum cuspidatum $=$ Tortula acaulon

Phascum cuspidatum var. piliferum $=$ Phascum piliferum

Philonotis arnellii $=$ Philonotis capillaris

Philonotis fontana subsp. capillaris $=$ Philonotis capillaris

Philonotis fontana var. pumila $=$ Philonotis tomentella

Physcomitrium acuminatum $=$ Physcomitrium eurystomum subsp. acuminatum

Physcomitrium sphaericum var. eurystomum $=$ Physcomitrium eurystomum

Plagiobryum zieri $=$ Ptychostomum zieri

Plagiopus oederi $=$ Plagiopus oederianus

Plagiotheciella latebricola $=$ Plagiothecium latebricola

Plagiotheciella pilifera $=$ Plagiothecium piliferum

Plagiothecium laetum var. curvifolium = Plagiothecium curvifolium

Plagiothecium neglectum $=$ Plagiothecium nemorale

Plagiothecium noricum $=$ Plagiothecium neckeroideum

Plagiothecium roeseanum $=$ Plagiothecium cavifolium

Plagiothecium ruthei $=$ Plagiothecium denticulatum var. undulatum

Plagiothecium ruthei var. rupicola = Plagiothecium platyphullum

Plagiothecium silvaticum $=$ Plagiothecium nemorale

Platydictya confervoides $=$ Serpoleskea confervoides

Platydictya subtilis $=$ Pseudoamblystegium subtile

Platyhypnidium riparioides $=$ Rhynchostegium riparioides 
Pleuridium nitidum $=$ Pseudephemerum nitidum

Pleuridium palustre $=$ Cleistocarpidium palustre

Pleurochaete squarrosa = Tortella squarrosa

Pogonatum alpinum = Polytrichastrum alpinum

Pohlia acuminata $=$ Pohlia elongata

Pohlia ambigua $=$ Pohlia elongata

Pohlia delicatula $=$ Pohlia melanodon

Pohlia elongata var. minor = Pohlia elongata var. greenii

Pohlia gracilis $=$ Pohlia fílum

Pohlia longicollis $=$ Pohlia longicolla

Pohlia minor $=$ Pohlia elongata

Pohlia rothii $=$ Pohlia fílum

Pohlia schleicheri $=$ Pohlia fílum

Polytrichastrum formosum = Polytrichum formosum

Polytrichastrum longisetum $=$ Polytrichum longisetum

Polytrichastrum pallidisétum $=$ Polytrichum pallidisetum

Polytrichum alpestre $=$ Polytrichum strictum

Polytrichum commune var. perigoniale $=$ Polytrichum perigoniale

Polytrichum commune var. swartzii = Polytrichum swartzii

Polytrichum decipiens $=$ Polytrichum pallidisetum

Polytrichum gracile $=$ Polytrichum longisetum

Polytrichum hercynicum $=$ Oligotrichum hercynicum

Polytrichum sexangulare $=$ Polytrichastrum sexangulare

Polytrichum uliginósum $=$ Polytrichum swartzii

Polytrichum undulatum $=$ Atrichum undulatum

Polytrichum undulatum var. minus $=$ Atrichum crispulum

Pottia bryoides $=$ Tortula protobryoides

Pottia davalliana $=$ Microbryum davallianum

Pottia intermedia $=$ Tortula caucasica

Pottia lanceolata $=$ Tortula lindbergii

Pottia randii $=$ Tortula randii

Pottia starckeana $=$ Microbryum starckeanum

Pottia truncata $=$ Tortula truncata

Pottia truncatula $=$ Tortula truncata

Protobryum bryoides $=$ Tortula protobryoides

Pseudocalliergon lycopodioides $=$ Drepanocladus lycopodioides

Pseudocalliergon trifarium $=$ Drepanocladus trifarius

Pseudoleskea incurvata $=$ Lescuraea incurvata

Pseudoleskea patens $=$ Lescuraea patens

Pseudoleskea catenulata $=$ Pseudoleskeella catenulata

Pseudoleskea patens $=$ Lescuraea patens

Pseudoleskea radicosa $=$ Lescuraea radicosa

Pseudoleskea saviana $=$ Lescuraea saviana

Pseudoleskeella nervosa var. rupestris $=$ Pseudoleskeella rupestris

Pterygoneurum cavifolium var. crossidioides $=$ Pterygoneurum crossidioides

Pterygoneurum kozlovii $=$ Lazarenkia kozlovii

Pterygoneurum pusillum $=$ Pterygoneurum ovatum

Pterogonium gracile $=$ Nogopterium gracile

Ptychodium plicatum $=$ Lescuraea plicata

Ptychodium tauricum $=$ Lescuraea saviana

Racomitrium canescens var. intermedium $=$ Racomitrium elongatum 
Racomitrium canescens var. ericoides $=$ Racomitrium ericoides

Racomitrium heterostichum var. affine $=$ Racomitrium affine

Racomitrium heterostichum var. sudeticum $=$ Racomitrium sudeticum

Racomitrium hypnoides $=$ Racomitrium lanuginosum

Racomitrium protensum $=$ Racomitrium aquaticum

Rhabdoweisia denticulata $=$ Rhabdoweisia crispata

Rhabdoweisia striata $=$ Rhabdoweisia fugax

Rhytidiadelphus calvescens $=$ Rhytidiadelphus subpinnatus

Schistidium anodon $=$ Grimmia anodon

Schistidium apocarpum var. dupretii $=$ Schistidium dupretii

Schistidium apocarpum subsp. papillosum $=$ Schistidium papillosum

Schistidium apocarpum var. rivulare $=$ Schistidium rivulare

Scleropodium purum = Pseudoscleropodium purum

Seligeria pusilla var. brevifolia $=$ Seligeria brevifolia

Sphagnum acutifolium $=$ Sphagnum capillifolium

Sphagnum denticulatum $=$ Sphagnum auriculatum

Sphagnum dusenii = Sphagnum majus

Sphagnum fimbriatum var. strictum = Sphagnum girgensohnii

Sphagnum nemoreum $=$ Sphagnum capillifolium

Sphagnum plumulosum $=$ Sphagnum subnitens

Sphagnum recurvum subsp. balticum $=$ Sphagnum balticum

Sphagnum recurvum var. amblyphyllum = Sphagnum flexuosum

Sphagnum recurvum var.mucronatum = Sphagnum fallax

Sphagnum recurvum var.obtusum $=$ Sphagnum obtusum

Sphagnum recurvum var. recurvum $=$ Sphagnum fallax

Sphagnum robustum $=$ Sphagnum russowii

Sphagnum subsecundum var. inundatum $=$ Sphagnum inundatum

Sphagnum tabulare $=$ Sphagnum molle

Syntrichia desertorum $=$ Syntrichia caninervis

Syntrichia inermis $=$ Tortula inermis

Syntrichia mucronifolia = Tortula mucronifolia

Syntrichia pulvinata = Syntrichia vírescens

Syntrichia ruralis var. arenicola $=$ Syntrichia ruraliformis

Syntrichia ruralis var. calcicola $=$ Syntrichia calcicola

Syntrichia subulata $=$ Tortula subulata

Tayloria serrata var. tenuis $=$ Tayloria tenuis

Taxiphyllum depressum $=$ Taxiphyllum wissgríllii

Thamnium alopecurum $=$ Thamnobryum alopecurum

Thuidium abietinum $=$ Abietinella abietina

Thuidium minutulum = Pelekium minutulum

Thuidium philibertii $=$ Thuidium assímile

Tortella caespitosa $=$ Tortella humilis

Tortula acaulon var. pilifera $=$ Phascum piliferum

Tortula calcicolens $=$ Syntrichia calcicola

Tortula caninervis $=$ Syntrichia caninervis

Tortula handelii $=$ Syntrichia handelii

Tortula lanceola $=$ Tortula lindbergii

Tortula latifolia $=$ Syntrichia latifolia

Tortula modica $=$ Tortula caucasica

Tortula montana $=$ Syntrichia montana

Tortula muralis var. aestiva = Tortula aestiva 
Tortula norvegica $=$ Syntrichia norvegica

Tortula papillosa $=$ Syntrichia papillosa

Tortula ruraliformis $=$ Syntrichia ruraliformis

Tortula ruralis $=$ Syntrichia ruralis

Tortula ruralis var. ruraliformis $=$ Syntrichia ruraliformis

Tortula sinensis $=$ Syntrichia sinensis

Tortula subulata var. angustata $=$ Tortula schimperi

Tortula virescens $=$ Syntrichia virescens

Syntrichia pulvinata = Syntrichia virescens

Trichostomum brevifólium $=$ Trichostomum crispulum

Trichostomum lanuginosum = Racomitrium lanuginosum

Trichostomum mutabile $=$ Trichostomum brachydontium

Trichostomum subulatum $=$ Ditrichum subulatum

Trichostomum tenuirostre $=$ Oxystegus tenuirostris

Trichostomum viridulum $=$ Trichostomum crispulum

Thuidium abietinum = Abietinella abietinum

Thuidium minutulum $=$ Pelekium minutulum

Ulota americana $=$ Ulota hutchinsiae

Ulota ludwigii = Ulota coarctata

Ulota crispa var. norvegica = Ulota bruchii

Warnstorfia exannulata $=$ Sarmentypnum exannulatum

Warnstorfia sarmentosa $=$ Sarmentypnum sarmentosum

Weisia fallax $=$ Weissia controversa var. crispata

Weisia microstoma $=$ Weissia brachycarpa

Weisia rupestris $=$ Gymnostomum aeruginosum

Weisia tortilis $=$ Weissia condensa

Weisia viridula $=$ Weissia controversa

Zygodon viridissimus var. rupestris = Zygodon rupestris

\section{References}

ANDREEVA E.N. (2012). Chornomorsk. bot. z., 8 (1): 49-55. [АНДРеЕВА Е.Н. (2012). Новые виды и находки рода Riccia (Marchantiophyta) для бриофлоры Украины. Чорноморськ. бот. ж., 8 (1): 49-55]

BACHURYNA H.F., MEL'NYCHUK V.M. (1988). Flora mokhiv Ukrayinskoi RSR. Vyp. 2. Kyiv: Nauk. dumka. 180 p. [БАчУРИНА Г.Ф., МЕЛЬНИчУК В.М. (1988). Флора мохів Української РСР. Вип. 2. Київ: Наук. думка. 180 с.]

BACHURYNA H.F., MEL'NYCHUK V.M. (2003). Flora mokhiv Ukrainy. Vyp. 4. Kyiv: Akademperiodyka. 255 p. [БАчУРИНА Г.Ф., МЕЛЬНичук В.М. (2003). Флора мохів України. Вип. 4. Київ: Академперіодика. 255 с.]

BAKAlin V.A., DumORT.S. (2005). str. The monographic study of Lophozia (Dumort.) Dumort. s. str. Moscow: Nauka. 1-238.

BAKalin V.A., Vilnet A.A. (2012). New combinations and new species of Solenostoma and Plectocolea (Solenostomataceae) from the Russian Far East. Bryologist, 115 (4): 566-584.

BARSUKOV O.O. (2014). Chornomors'k. bot. z., 10 (3): 305-321. [БАРСУКОВ О.О. (2014). Бріофлора урбанізованих територій Харківської області. Чорноморськ. бот. ж., 10 (3): 305-321]

BARSUKOV O.O. (2014). Zb. tez dop. Mizhnar. nauk. konf. (Kherson, 19.05.2014 - 22.05.2014) / vidp. red. M.F. Boiko. Kherson: Ailant. 22-23. [БАРсуков О.О. (2014). Мохоподібні заболочених місцезростань Харківщини. VI Ботанічні читання памяті Й.К.Пачоського. Зб. тез доп. Міжнар. наук. конф. (Херсон, 19.05.2014 - 22.05.2014) / відп. ред. М.Ф.Бойко. Херсон: Айлант. 22-23]

BARSUKOV O.O. (2015). Chornomors'k. bot. z. (in press). [БАРСукОВ О.О. (2015). Локально рідкісні мохоподібні Харківщини, їх особливості та стан охорони. Чорноморськ. бот. ж. (в друці)]

BARSUKOV O.O., VIRCHENKO V.M. (2012). Ukr. botan. zhurn., 69 (6): 880-885. [БАРСУКОВ О.О., Вірченко B.M. (2012). Myrinia pulvinata (Wahlenb.) Schimp. (Amblystegiaceae) - новий вид і рід для бріофлори України. Укр. ботан. журн., 69 (6): 880-885]

Bell, N.E. \& Hyvonen, J. (2010). A phylogenetic circumscription of Polytrichastrum (Polytrichaceae): reassement of sporophyte morphology suppors molecular phylogeny. American Journal of Botany, 97 (4): 566-578. 
Blom H.H. (2005). A revision of the Schistidium apocarpum complex in Norway and Sweden. Bryoph. Biblith., 49: 1-333.

BoІко M.F. (2011A). Chornomors'k. bot. z., 7 (2): 144-186. [Бойко М.Ф. (2011a). Анотований список мохоподібних Лівобережного Полісся (Україна). Чорноморськ. бот. ж., 7 (2): 144-186]

BоIко M.F. (2004). Chornomors'k. bot. z., 5 (4): 583-588. [БОйко М.Ф. (2004). Анотований список мохоподібних заповідника «Єланецький степ» та прилеглих територій (Миколаївська область, Україна). Чорноморськ. бот. ж., 5 (4): 583-588]

BоІко M.F. (2008). Checklist mokhopodibnykh Ukrayiny. Kherson: Aylant. 229 с. [БОЙКО М.Ф. (2008). Чекліст мохоподібних України. Херсон: Айлант. 229 с.]

Boiko M.F. (2009 в). Chornomors'k. bot. z., 5 (1): 23-27. [БойКо М.Ф. (2009 б). Матеріали до бріофлори Нижньобузьких пісків (Миколаївська область, Україна). Чорноморськ. бот. ж.,5 (1): 23-27]

BoІко M.F. (2009). Mokhopodibni stepovoi zony Ukrayiny. Kherson: Aylant. 264 p. [БойКО М.Ф. (2009). Мохоподібні степової зони України. Херсон: Айлант. 264 с.]

Bогко M.F. (2010A). Chornomors'k. bot. z., 6 (1): 95-101. [БОЙКО М.Ф. (2010А). Aulacomnium arenopaludosum Boiko sp.nov. (Bryopsida, Aulacomniaceae) - новий вид мохів зі степової зони України. Чорноморськ. бот. ж., 6 (1): 95-101.

BoІко M.F. (2010в). Chervonyi spysok mokhopodibnykh Ukrayiny. Kherson: Aylant. 94 p. [БОЙКО М.Ф. (2010b). Червоний список мохоподібних України. Херсон: Айлант. 94 с.]

BoIKo M.F. (2011 b). Syntrichia ruralis var. polysporogonica and Riccia rhenana var. violacea - new infraspecific bryophytes taxa. Chornomorsk. bot. z., 7 (1): 92-93.

BoIKo M.F. (2011). Lazarenkia (Pottiaceae, Bryopsida) - new genus of mosses and Lazarenkia kozlovii - new combination at the species rank. Chornomorsk. bot. z., 7 (4): 400-402.

Boiко M.F. (2012). Chornomors'k. bot. z., 8 (2): 475-462. Бойко М.Ф. (2012). Доповнення до Чекліста мохоподібних України. Чорноморськ. бот. ж., 8 (2): 475-462.

BOLYUKH V.O. (1995). Ukr. botan. zhurn., 52 (4): 536-541. [БолЮХ В.О. (1995). Рідкісні мохоподібні центральної частини Подільської височини. Укр. ботан. журн., 52 (4): 536-541]

Boros A. (1951). Bryologische Beitrage zur Kenntnis der Flora von Ungarn und der Karpaten. Acta Biol. Acad. Sci. Hung., $2(3 / 4): 369-409$.

Boros A., VAJDA L. (1969). Bryoflora Carpathorum Septentrionali-Orientariorum.- Revue Bryol. Et Lichen, 36 (3-4): 397-450.

Borovichev E.A., NyPORKO C.A. (2014). Ukr. botan. zhurn., 71 (1): 66-69. [БОРОВИЧЕВ Е.А., НЫПОРКО С.А. (2014). Три таксона из семейства Aitoniaceae, новые для флоры печеночников Украины. Укр. ботан. журн., 71 (1): 66-69]

BRYOPHYTE biology.(2009). [edited by] Bernard Goffinet \& A.Jonathan Shaw. $-2^{\text {nd }}$ ed. Cambrydge: Cambrydge University Press. 565 p.

Buck, W. R., Goffinet, B. and SHAW, A., J. (2000). Novel relationships in pleurocarpous mosses as revealed by cpDNA sequences. Bryologist, 103: 774-789.

CHECKLIST and country status of European bryophytes -towards a new Red List for Europe / N. G. Hodgetts, 2014 _ version 1. pdf http://eccbbryo.nhmus.hu/node/4.

Chervona knyha Ukrayiny. Roslynnyi svit (1996). red. Yu.R. Shelyah-Sosonko. Kyiv: Ukr. entsyklopedia. 608 p. [ЧервонА книга України. Рослинний світ (1996). ред. Ю.Р.Шеляг-Сосонко. Київ: Укр. енциклопедія. 608 с.]

CHERvona knyha Ukrayiny. Roslynnyi svit (2009). red. Ya.P. Didukh. K.: Hlobalkonsaltynh. 912 p. [ЧЕРвOHA книга України. Рослинний світ (2009). ред. Я.П. Дідух. К.: Глобалконсалтинг. 912 с.]

Č́́HAL L. (2014). Contribution to the occurrence of mosses from Orthotrichum and Nyholmiella genera in Crimean Peninsula (Ukraine). Acta Mus. Siles. Sci. Natur., 63: 55-59.

Cox C.J. \& Hedderson T.A.J. (2003). Phylogenetic relationships within the moss family Bryaceae based on chloroplast DNA evidence. J. Bryol., 25 (1): 31-40.

Crandall-Stotler, B., Stotler R.E. \& Long D.G. (2009). Phylogeny and classification of the Marchantiophyta. Edinburgh J. Bot., 66: 155-198.

Crandal-Stotler, B.J., Stotler R.E. \& Long D.G. (2008). Morphology and classification of the Marchantiophyta - In: Goffinet, B. \& A.J. Show (eds.) Bryophyte Biology, 2 ed. Cambridge. 1-54.

Crosby M.R., Magil R. E., Allen B., He S. (1999). A checklist of the mosses. - St Louis: Missuri Botanical Garden. 319 p.

Crosby, M.R. \& Buck, W.R. (2011). Nogopterium, a new name for the genus Pterogonium (Musci, Leucodontaceae). Novon, 21: 424-425.

Danilkiv I.S., Ignatova E.A., Lobachevska O.V. (2009). Chromosome numbers of schistidium (Grimmiaceae, Bryophyta). Arctoa, 18: 225-228.

Danylkiv I.S., Demkiv O.T., Lobachevs'Ka O.V., Mamchur Z.I. (1997). Mokhopodibni - Bryophyta. Bioriznomanittia Karpatskoho biosfernoho zapovidnyka. Kyiv: Interekotsentr. 190-198, 576-592. [ДАНИЛКІв І.С., ДЕМКІв О.Т., ЛОБАЧЕВСьКА О.В., МАмчУР 3.I. (1997). Мохоподібні - Bryophyta. 
Біорізноманіття Карпатського біосферного заповідника. Київ: Інтерекоцентр. 190-198, 576592]

DanYlkiv I.S., Lobachevs'Ka O.V., MamchUR Z.I., Soroka M.I. (2002). Mokhopodibni Ukrayinskoho Roztochchia. Lviv. 320 p. [ДАНИЛКІВ I.C., ЛОБАЧЕВСьКА O.B., МАМчур 3.I., СОРОКА M.I. (2002). Мохоподібні Українського Розточчя. Львів. 320 с.]

DÜLL R. (1983). Distribution of the European and Macaronesian Liverworts (Hepaticopytina). Bryologische Beitrage, 2: 1-115.

Ellis T., Bednarec-Ochyra H., Cykowska B., Ochyra R. et al. (2012). New national and regional bryophytes recoreds, 30. Journal of Bryology, 34 (1): 45-51.

FeldBerg, K., HeINRICHS, J., SCHMIDT, A.R., VAŇA, J. \& SCHNEIDER, H. (2013). Exploring the impact of fossil constraints on the divergence time estimates of derived liverworts. Plant Systematics and Evolution. 299: 585-601. http:// dx.doi. org/10.1007/s00606-012-0745-y

Feldberg, K., VÁŇA, J., Hentschel, J. \& Heinrichs, J. (2010). Currently accepted species and new combinations in Jam esonielloideae (Adelanthaceae, Jungermanniales). Cryptogamie, Bryologie, 31: 141-146.

GAPON S.V. (1998). Anotovanyi spysok mokhopodibnykh. Bezsudynni roslyny Livoberezhnoho Lisostepu Ukrainy. Poltava: Verstka. 98-130. [ГАПОН С.В. (1998). Анотований список мохоподібних. Безсудинні рослини Лівобережного Лісостепу України. Полтава: Верстка. 98-130]

GAPON S.V. (2011). Mokhopodibni Lisostepu Ukrainy (roslynnist ta flora). Avtoref. dys.. .. dokt. biol.. nauk. 03.00 .05 - botanika. Kyiv. 36 p. [ГАПОН С.В. (2011). Мохоподібні Лісостепу України (рослинність та флора). Автореф. дис.. .. докт. біол.. наук. - 03.00.05 - ботаніка. Київ. 36 с.]

GofFINET B., BUCK W.R. (2004). Systematics of the bryophyte (mosses): from molecules to a revised classification. In: Goffinet B., Hollowel V.C., Magill R.E., eds. Molecular systematics of bryophytes. - St Louis: Missuri Botanical Garden Press. 205-223.

Goffinet, B., Shaw, A.J., Cox, C.J., Wickett, N.J. \& Boles, S. (2004). Phylogenetic inferences in the Orthotrichoideae (Orthotrichaceae: Bryophyta) based on variation in four loci from all genomes. Monographs in Systematic Botany from the Missouri Botanical Garden, 98: 270-289.

Gofinet B., BuCK W.R., Shaw A.J. (2008). Morphology, anatomy and classification of the Bryophyta. In: Goffinet, B. \& A.J. Show (eds.) Bryophyte Biology, 2 ed. Cambridge. 55-138.

Greven H.C. (1995). Grimmia Hedw. (Grimmiaceae, Musci) in Europe.- Leiden: Backhuys Publishers. 1995. $146 \mathrm{p}$.

Grundmann, M., Schneider, H., Russell S.J. \& Vogel, J.C. (2006). Phylogenetic relationships of the moss genus Pleurochaete Lindb. (Bryales: Pottiaceae) based on chloroplast and nuclear genomic markers. Organisms diversity and evolution, 6: 33-45.

Hedenas, L. \& Rosborg, C. (2008). Pseudocalliergon is nested within Drepanocladus (Bryophyta: Amblystegiaceae). Lindbergia, 33: 67-74.

Hedenäs, L. (2006). Additional insights into the phylogeny of Calliergon, Loeskypnum, Straminergon and Warnstorfia (Bryophyta: Calliergonaceae). J. Hatt. Bot. Lab., 100: 125-134.

HEDENS, L. (2001). Environmental factors potentially affecting character states in pleurocarpous mosses. Bryologist, 104: 72-91.

Hentschel J., Paton J.A., H. Schneider \& Heinrichs J. (2007). Acceptance of Liochlaena Nees and Solenostoma Mitt., the systematic position of Eremonotus Pearson and notes on Jungermannia L. s.l. (Jungermanniidae) based on chloroplast DNA sequence data. Pl. Syst. Evol., 268: 147-157.

He-Nygren X., Juslen A., GlenNy D. \& PiIPPo S. (2006). Illuminating the evolutionary history of liverworts (Marchantiophyta) - towards a natural classification. Cladistics, 22: 1-31.

Hill, M.O., Bell, N., BRUGgeman-NANNEnga et al. An annotated checklist of the mosses of Europe and Macaronesia. Journal of Bryology, 28: 198-267.

Hill, M.O., Blackstock T.H., Long D.G. \& Rothero G.P. (2008). A Checklist and Census Catalogue of British and Irish Bryophytes. BBS. 1-184.

HolyoAK, D.T. \& PeDERSEN, N. (2007). Conflicting molecular and morphological evidence of evolution within the Bryaceae (Bryopsida) and its implications for generic taxonomy. Journal of Bryology, 29: 111124.

HolyoAK, D.T. (2010). Notes on taxonomy of some European species of Ephemerum (Bryopsida: Pottiaceae). Journal of Bryology, 32: 122-132.

HutTUnEN S. (2004). Phylogeny and evolutionary relationships of Brachytheciaceae and Meteoriaceae. Publications in Botany from the University of Helsinki, 34: 1-33.

Huttunen S. and Ignatov M.S. (2004). Phylogenetic analyses of Brachytheciaceae (Bryophyta) based on morphology, and sequence level data. Cladistics, 4 (20): 151-183.

Huttunen, S. \& Ignatov, M.S. (2010). Evolution and taxonomy of aquatic species in the genus Rhynchostegium (Brachytheciaceae, Bryophyta). Taxon, 59: 791-808.

Ignatov M. S., Milutina I.A. (2007). On Sciuro-hypnum oedipódium and Sciuro-hypnum curtum (Brachytheciaceae, Bryophyta). Arctoa, 16: 47-61. 
IgNATOv M.S. and HutTunEN S. (2002). Brachytheciaceae (Bryophyta) - family of sibling genera. Arctoa, 11: 229-244.

IgnAtov M.S., AfOnINA O.M. (1992). (eds.). Check-list of the mosses of the former USSR. Arctoa, 1:1-85.

IgnAtova E.A., Seregin A.P. (2007). New moss records from the Autonomous Republik of Crimea, Ukraine. Arctoa, 16: 209-210.

INTERNET resources. http://www.catalogueoflife.org/annual-checklist/2012/details/species/id/7135747 Ukraine \& Moldova Catalogue of Life: 2012 Annual Checklist.

IUCN 2014. Guidelines for using the IUCN Red List categories and criteria. Version 11 (February 2014). Gland, International Union for the Conservation of Nature.

Konstantinova N.A. \& Vilnet A.A. (2009). New taxa and new combinations in Jungermanniales (Hepaticae). Arctoa, 18: 65-67.

Konstantinova N.A., Bakalin V.A., Andreeva E.N., Bezgodov A.G., Borovichev E.A., Dulin M.V., Mamontov Yu.S. (2009). Checklist of liverworts (Marchantiophyta) of Russia. Arctoa, 18: 1-63.

Koponen, T. \& IsovittA, P. (2010). Philonotis capillaris Lindb. and P. arnellii Husn.; one moss, two names. Cryptogamie, Bryologie, 31: 75-94.

KRUPA I. (1885). Zapyski bryologiczne z okoloc Lwowa, Krakowa i Wschodnich Karpat. Spraw. komis. Fizijogr, 19: 133-167.

KuČERA J., VÁŇA J. (2009). Chek- and Red List of bryophytes of the Czech Republic. Preslia, 75: $193-222$.

KUZYARIN O.T. (2012). Ukr. botan. zhurn., 69 (3): 16-22. [КузяРін О.Т. (2012). Нові відомості про поширення адвентивного моху Campylopus introflexus (Hedw.) Brid. в Україні. Укр. ботан. журн., 69 (3): 16-22]

LAZARENKO A.S. (1955). Opredelytel listvennykh mkhov Ukrainy. Kiev: Yzd-vo AN USSR. 466 p. [ЛАЗАРЕНКО A.С. (1955). Определитель лиственных мхов Украины. Киев: Изд-во АН УССР. 466 с.]

LAZARENKO A.S., VySOTSKAIA E.Y., LESNYAK E.N. (1971). Atlas khromosom listvennykh mkhov SSSR. Kiev: Nauk. dumka. 143 р. [ЛАЗАРЕНКО А.С., ВЫСОЦКАЯ Е.И., ЛЕСНЯК Е.Н. (1971). АтЛас хромосом лиственных мхов СССР. Киев: Наук. думка. 143 с.]

LEWINSKY J. (1993). A synopsis of the genus Orthotrichum Hedw. (Orthotrichaceae, Musci). - Bryobrothera, 2. $1-59$.

LOBACHEVSKA O.V., GAPON S.V. (1988). Ukr. botan. zhurn., 45 (3): 49-52. [ЛОБАЧЕВСЬКА О.В., ГАПОН С.В. (1988). Хромосомні числа листяних мохів України. Укр. ботан. журн., 45 (3): 49-52]

LOBACHEVSKa O.V., SOKHANCHAK R.R. (2010). Ukr. botan. zhurn., 67 (3): 432-438. [ЛОБАЧЕВСьКА О.В., СОХАНЬЧАК P.P. (2010). Campylopus introflexus (Hedw.) Brid. - адвентивний вид моху для флори України. Укр. ботан. журн., 67 (3): 432-438]

MELNYCHUK V.M. (1970). Opredelitel listvennykh mhov srednei polosy i yuga Evropeyskoi chasti SSSR. Kiev: Nauk. dumka. 442 p. [МЕльничук B.M. (1970). Определитель лиственных мхов средней полосы и юга Европейской части СССР. Киев: Наук. думка. 442 с.]

MunOz J. \& PANDo F. (2000). A word synopsis of the genus Grimmia (Musci: Grimmiaceae). St. Luis: Missour Bot. Gard. 85. 133 p.

Nyporko S.O., PARTYKa L. JA. (2011). Annotated list bryophytes of granitic canyons // Annotated list lichenforming, bryophytes and terrestrial algae of granitic canyons of Ukraine. Kyiv: Alterpres. 342-347.

Ochyra, R., Zarnowiec, J. \& Bednarek-Ochyra, H. (2003). Census Catalogue of Polish mosses. Krakow, Polish Academy of Sciences, W. Szafer Institute of Botany.

Olsson, S., Enroth, J., Buchbender, V., Hedenas, L., Huttunen, S. \& Quandt, D. (2011). Neckera and Thamnobryum (Neckeraceae, Bryopsida) - paraphyletic assemblages. Taxon, 60: 36-50.

PARTYKA L.YA. (2005). Brioflora Kryma. Kiev: Fitosotsiotsentr. 170 р. [ПАРТЫКА Л.Я. (2005). Бриофлора Крыма. Киев: Фитосоциоцентр. 170 с.]

PARTYKA L.YA., ВОIКо M.F. (1980). Ukr. botan. zhurn., 37 (4): 85-88. [ПАРТИКА Л.Я., БОЙКО М.Ф. (1980). Бріофлора урочища Лиса гора (м. Київ). Укр. ботан. журн., 37 (4): 85-88]

PARTYKA L.YA., SlOBODYAN M.P. (1989). Ukr. botan. zhurn., 45 (3): 38-41. [ПАРТИКА Л.Я., СЛОБОДЯН М.П. (1989). Нові для бріофлори Криму види мохоподібних. Укр. ботан. журн., 45 (3): 38-41]

Plašek, V., SAWicki, J., Markova, I. \& Wierzcholska, S. (2011). Orthotrichum affine var. bohemicum (Orthotrichaceae), a new variety of epiphytic moss from the Czech Republic. Acta Societatis Botanicorum Poloniae, 80: 335-340.

Potemkin A.D. (2002). Phylogenetic system and classification of the family Scapaniaceae Mig. emend. Potemkin (Hepaticae). Ann. Bot. Fennici., 39: 309-334.

RABYK I.V., DANYLKIV I.S. (2004). Mokhopodibni (Bryophyta) ta antotserotopodibni (Anthocerothophyta) Ukrayinskoho Roztochchia. Y.K. Pachoskyi ta suchasna botanika (Vidp. red. M.F. Boiko). Kherson: Aylant. 66-73. [РАБИк I.В., ДАнилкІв І.С. (2004). Мохоподібні (Bryophyta) та антоцеротоподібні (Anthocerothophyta) Українського Розточчя. Й.К. Пачоський та сучасна ботаніка (Відп. ред. М.Ф. Бойко). Херсон: Айлант. 66-73]

RABYK I.V., DANYLKIV I.S. (2008). Ridkisni dlia Ukrainy vydy mokhopodibnykh (Hepaticophyta, Bryophyta) bolota Nemyriv (Lvivska obl.). Znachennia ta perspektyvy statsionarnykh doslidzhen dlia 
zberezhennia bioriznomanittia: Mat-ly mizh nar. nauk. konf.- Lviv-Pozhyzhevska, 23-27 veresnya 2008. Lviv. 348-349. [РАБик І.В., ДАнилків I.C. (2008). Рідкісні для України види мохоподібних (Hepaticophyta, Bryophyta) болота Немирів (Львівська обл.). Значення та перспективи стаціонарних досліджень для збереження біорізноманіття: Мат-ли між нар. наук. конф.- ЛьвівПожижевська, 23-27 вересня 2008 р. Львів. 348-349]

Rahulina M.Ye., Isikov V.P. (2012). Chornomors'k. bot. z., 8 (2): 240-243. [РАГУліна М.Є., Ісіков В.П. (2012). Syntrichia laevipila Brid. - новий вид моху для флори України. Чорноморськ. бот. ж., 8 (2): 240-243]

REJMENT-GROCHOWSKA J. (1958). WAtrobowce Bieszad i niekotorych innych czesci Karpat Wschodnich w zbirach J. Lobazewskiego. Acta Soc.Bot. Poloniae, XXVII (2).

ReNZAGLia K.S., VillarReAl J.C., DufF R.J. (2008). New insights into morphology, anatomy and systematic of hornvorts. - In: Goffinet, B. \& A.J. Show (eds.) Bryophyte Biology, 2 ed. Cambridge. 139-171.

Roo De, R.T., HedDERSON, T.A. \& Soderstrom, L. (2007). Molecular insights into the phylogeny of the leafy liverwort family Lophoziaceae Cavers. Taxon, 56: 301-314.

Ros, R.M. \& HerRnSTADT, I. (2010). New taxonomical data in the genus Tortula (Pottiaceae, Bryophyta). Tropical bryology, 31:150-153.

Ros, R.M., Mazimpaka, V., Abou-Salama, U., Aleffi, M., Blockeel, T.L., Brugues, M., Cros, R.M., Dia, M.G., Dirkse, G.M., Draper, I., El-SaAdawi, W., ErdaĞ, A., Ganeva, A., Gabriel, R., González-Mancebo, J.M., Granger, C., Herrnstadt, I., Hugonnot, V., Khalil, K., Kurschner, H., Losada-Lima, A., Luís, L., Mifsud, S., Privitera, M., Puglisi, M., Sabovljević, M., Sergio, C., Shabbara, H.M., Sim-Sim, M., SotiauX, A., Tacchi, R., Vanderpoorten, A. \& WERnER, O. (2013). Mosses of the Mediterranean, an annotated checklist. Cryptogamie, Bryologie, 34: 99-283.

Ros, R.M., MunOz, J., Werner, O. \& RAms, S. (2008). New typifications and synonyms in Tortula sect. Pottia (Ehrh. ex Rchb.) Kindb. (Pottiaceae, Musci). Taxon, 57: 279-288.

RUBASINGHE S.C.K., LONG D.G. \& MiLNE R. (2011A). A new combination and three new synonyms in the genus Clevea Lindb. (Marchantiopsida, Cleveaceae). Journal of Bryology, 33 (2): 168-169.

RubAsinghe, S.C.K., R. MiLNE, L.L. ForREST \& D.G. Long 2011b. Realignment of the Genera of Cleveaceae (Marchantiopsida, Marchantiida). The Bryologist, 114: 116-127.

SCHUMACKER R. \&. VAŇA J. (2005). Identification keys to the liverworts and hornworts of Europe end Macaronesia (distribution and status). Poznan, Sorus. 1-212.

SeneCa A., SöDerström L. (2009). Sphagnophyta of Europe and Macaronesia: a checklist with distribution data. Journal of Bryology, 31: 243-254.

Slobody AN M.P. (1950). Botan. zhurn. AN URSR, 7 (2): 80-82. [Слободян М.П. (1950). Бріофлористичні новинки з Свидівця (Східні Карпати). Ботан. журн. АН УРСР, 7 (2): 80-82]

SÖDERSTRÖM L., De Roo R., HEDDERSOn T. (2010). Taxonomic novelties resulting from recent reclassification of the Lophoziaceae/Scapaniaceae clade. Phytotaxa, 3: 47-53.

SÖDERStröm L., Hagborg A., Crosby M. R. \& Konrat M. von. EARLy (2012). Land Plants Today: Index of Liverworts \& Hornworts 2009-2010. Phytotaxa, 63: 21-68 (2012) www.mapress.com/phytotaxa.

SöDERSTRÖM L., URMi E AND VÁÑa J. (2002). Distribution of Hepaticae and Anthocerotae in Europe and Macaronesia. Lindbergia, 27: 3-47.

StefuREAK G. (1936). Consideration asupra vegetatici si florei muchilor (Bryophytes) din Vales Vijnicioarei (Bucovina). Bull. Facult. de Stiinte in Cernauti, 5 (3): 8-15.

SZWEYKowsKi J., BuCZKOWSKA, ODRZYKOSKI I.J. (2005). Conocephalum salebrosum (Marchantiopsida, Conocephalaceae) - a new holarctic liverwort species. Plant Systematics and Evolution, 253:133-158.

Tsubota H., Arikawa T., Akiyama H., Luna E.De, Gonzales D., Higuchi M., Deguchi H. (2002). Molecular phylogeny of hypnobryalean mosses as inferred from the large scale dataset of chloroplast rbcL., with special reference on the Hypnaceae and possibly related families. Hikobia, 13. 645-665.

VÁŇA J., Söderström L., Hagborg A., KonRat M. von. (2013). Notes on Early Land Plants Today. 33. Notes on Anastrophyllaceae (Marchantiophyta). Phytotaxa, 81 (1): 26-32. www.mapress.com/phytotaxa/

VÁÑa J., SöDerström L., Hagborg A., von KonRat M. (2012). Notes on Early Land Plants Today. 8. New combinations and some lectotypifications in Mesoptychia. Phytotaxa, 6. 52-56.

VÁÑa J., Söderström L., Hagborg A., von KonRat M. (2013). Notes on Early Land Plants Today. 41. New combinations and synonyms in Cephaloziaceae (Marchantiophyta). Phytotaxa, 112 (1): 7-15.

VÁŇA J., VIRCHENKO V.M. (1993). Ukr. botan. zhurn., 50 (4): 83-93. [ВАНЯ I., ВІРчЕНКО В.М. (1993). Зведений список антоцеротів та печіночників України. Укр. ботан. журн., 50 (4): 83-93]

VAnderpoorten, A. \& HedenAs, L. (2009). New combinations in the Amblystegiaceae. Journal of Bryology, 31: $129-132$.

Vanderpoorten, A., Hedenäs, L., Cox, C. J. AND Shaw, A. J. (2002). Circumscription, classification, and taxonomy of the Amblystegiaceae (Bryopsida) inferred from nuclear and chloroplast DNA sequence data and morphology. Taxon, 51: 115-122. 
Vilnet A.A., Konstantinova N.A., Troitsky A.V. (2010). Molecular insight on phylogeny and systematics of the Lophoziaceae, Scapaniaceae, Gymnomitriaceae and Jungermanniaceae. Arctoa, 19: 31-50.

Vilnet A.A., KonstantinOva N.A., TROITSKY A.V. (2011). Taxonomical rearrangements of Solenostomataceae (Marchantiophyta) with description of a new family Endogemmataceae based on trnL-F cpDNA analysis. Folia Cryptogamica Estonica, 48: 125-133.

Vilnet A.A., Konstantinova N.A., Troitsky A.V. (2012). Molecular phylogeny and systematics of the suborder Cephaloziineae with special attention to the family Cephaloziaceae s.l. (Jungermanniales, Marchantiophyta). Arctoa, 21: 113-132.

VIRCHENKo V.M. (2000). Spysok bokoplidnykh mokhiv Ukrayiny. Kyiv: Znannia. 32 p. [ВірЧЕНКО B.M. (2000). Список бокоплідних мохів України. Київ: Знання. 32 с.]

VIRCHENKO V.M. (2001B). Spysok verkhoplidnykh mokhiv Ukrayiny. Kyiv: Znannia. 56 p. [ВIPЧEHKO B.M. (2001б). Список верхоплідних мохів України. Київ: Знання. 56 с.]

Virchenko V.M. (2005). Ukr. botan. zhurn., 62 (5): 715-718. [Вірченко В.M. (2005). Рід Zygodon Hook etr Taylor (Orthotrichaceae, Bryophyta) в Україні. Укр. ботан. журн., 62 (5): 715-718]

VIRCHENKo V.M. (2010). Visnyk Uzhhorods'koho universytetu, Seriya Biolohiia, 27: 85-87. [ВІРЧЕНКО В.М. (2010). Нові відомості про рід Рohlia в Україні. Наук. вісник Ужгородського університету, Серія Біологія, 27: 85-87]

VIRChEnKo V.M. (2012). Dichelyma capillaceum (Dicks.) Myrin (Bryophyta) - kandydat do «Chervonoi knyhy Ukrayiny». Rastytelnyi mir v Krasnoi knyhe Ukrainy: realyzatsiia hlobalnoi stratehii sokhraneniia rastenii. Mat. II Mezhdunar. nauchn. konf., 9-12 oktiabria 2012, Uman, Cherkasskaia oblast. 196197. [Вірченко B.M. (2012). Dichelyma capillaceum (Dicks.) Myrin (Bryophyta) - кандидат до «Червоної книги України». Растительный мир в Красной книге Украины: реализация глобальной стратегии сохранения растений. Мат. II Междунар. научн. конф., 9 - 12 октября 2012, Умань, Черкасская область. 196-197]

Virchenko V.M., MaLA YU.I. (2014). Novi ta ridkisni mokhopodibni dlia Krymu. VI Botanichni chytannia pamiati Y.K. Pachoskoho. Zb. tez dop. Mizhnar. nauk. konf. (Kherson, 19.05.2014 - 22.05.2014), vidp. red. M.F. Boiko. Kherson: Ailant. 25-26. [ВІРченкО В.М., МАЛА Ю.І. (2014). Нові та рідкісні мохоподібні для Криму. VI Ботанічні читання памяті Й.К.Пачоського. Зб. тез доп. Міжнар. наук. конф. (Херсон, 19.05.2014 - 22.05.2014), відп. ред. М.Ф.Бойко. Херсон: Айлант. 25-26]

VIRCHENKO V.M., ORlov O.O. (2011). Visnyk Zhyt. nats. ahrarn. un-ta, 1 (28): 314-322. [ВіРчEHКО В.М., Орлов О.О. (2011). Нові та рідкісні мохоподібні для Житомирської області. Вісник Жит. наu. аграрн. ун-та, 1 (28): 314-322]

Virchenko V.M., PARTyka L.YA. (2010). Mat-ly do brioflory Mezynskoho NPP. Mat-ly mizhn. nauk.-prakt. konf. «Bioriznomanittia: teoriya, praktyka ta metodychni aspekty vyvchennia u zahalnoosvitnii ta vyshchii shkoli». Poltava. 56-61. [ВІРчЕНко В.М., ПАРТИКА Л.Я. (2010). Матеріали до бріофлори Мезинського НПП. Мат-ли міжн. наук.практ. конф. «Біорізноманіття: теорія, практика та методичні аспекти вивчення у загальноосвітній та вищій школі». Полтава. 56-61]

VIRCHENKO V.M., VÁŇA J. (2000). Spysok pechinochnykiv, antotserotiv ta sfahnovykh mokhiv Ukrayiny. Kyiv: Znannia. 29 p. [ВІрченко B.M., ВАНЯ I. (2000). Список печіночників, антоцеротів та сфагнових мохів України. Київ: Знання. 29 с.]

VysotsKa O.I. (1984B). Ukr. botan. zhurn., 41 (5): 29-33. [ВиСОцьКА О.I. (1984б). Порівняльна характеристика Brachythecium starkei (Brid.) B.S.G. i B. curtum (Lindb.) Limpr. західної частини СРСР. Укр. ботан. журн., 41 (5): 29-33]

Werner, O., Patino, J., Gonzales-Mancebo, J.M. \& Ros, R.M. (2007). The taxonomic status of Platyhypnidium torrenticola based on ITS sequence data. Cryptogamie, Bryologie, 28: 187-195.

Werner, O., RAMS, S., Kučera, J., Larraín, J., Afonina, O.M., PisA, S. \& Ros, R.M. (2013). New data on the moss genus Hymenoloma, with special reference to H. mulahaceni. Cryptogamie, Bryologie, 34 (1): 13-30.

Werner, O., Ros, R.M. \& Grundmann, M. (2005). Molecular phylogeny of Trichostomoideae (Pottiaceae, Bryophyta) based on nrITS sequence data. Taxon, 54: 361-368.

Werner, O., Ros, R.M., CANO, M.J. \& Guerra, J. (2002). Tortula and some related genera (Pottiaceae, Musci): phylogenetic relationships based on chloroplast rps4 sequences. Plant systematics and evolution, 235: 197-207.

Werner, O., Ros, R.M., CANO, M.J. \& Guerra, J. (2004). Molecular phylogeny of Pottiaceae (Musci) based on chloroplast rps4 sequence data. Plant systematics and evolution, 243: 147-164.

Yatsentyuk, S.P., Konstantinova N.A., Ignatov M.S., Hyvonen J. \& Troitsky A.V. (2004). On phylogeny of Lophoziaceae and related families (Hepaticae, Jungermanniales) based on trnL-trnF intron-spacer sequences of chloroplast DNA. - In: (Goffinet B., V. Hollowell \& R. Magill eds.) Molecular Systematics of Bryophytes. Monogr. Syst. Bot. St. Louis, Missouri Bot. Gard. Press. 98. 150-167.

ZANDER R.H. (1993). Genera of the Pottiaceae: mosses of harsh environments. Bull. Buffalo Soc. Nat. Sci., 32 (1): $378 \mathrm{p}$. 
ZEROV D.K. (1964). Flora pechinochnykh i sfahnovykh mokhiv Ukrayiny. К.: Nauk. dumka. 355 p. [ЗЕРОВ Д.К. (1964). Флора печіночних і сфагнових мохів України. К.: Наук. думка. 355 с.]

ZEROv D.K., PARTYKA L.YA. (1975). Mokhopodibni Ukrainskykh Karpat. K.: Nauk. dumka. 230 p. [ЗЕРОВ Д.К., ПАРтиКА Л.Я. (1975). Мохоподібні Українських Карпат. К.: Наук. думка. 230 с.]

Рекомендує до друку

Отримано 15.11.2014

О.С.Ходосовцев

Aдpeca автора:

М.Ф. Бойко

Херсонський державний університет

вул. 40 років Жовтня, 27

Херсон 73000

Украӥна

e-mail:bomifed@ksu.ks.ua
Author's address:

M.F. Boiko

Kherson State University

27, 40 Rokiv Zhovtnia str.

Kherson, 73000

Ukraine

e-mail:bomifed@ksu.ks.ua 